\title{
Insertion of Cyclic Alkyl(Amino) Carbene into the Si-H Bonds of Hydrochlorosilanes
}

\author{
Chandrajeet Mohapatra, ${ }^{1}$ Prinson P. Samuel, ${ }^{1}$ Bin Li, ${ }^{1}$ Benedikt Niepötter, ${ }^{1}$ Christian J. Schürmann, ${ }^{1}$ \\ Regine Herbst-Irmer, ${ }^{1}$ Dietmar Stalke, ${ }^{1 *}$ Bholanath Maity, ${ }^{2}$ Debasis Koley ${ }^{2}$ and Herbert W. Roesky ${ }^{1 *}$ \\ ${ }^{1}$ Institut für Anorganische Chemie, Universität Göttingen, Tammannstrasse 4, 37077 Göttingen, Germany. \\ ${ }^{2}$ Department of Chemical Sciences, Indian Institute of Science Education and Research - Kolkata, India. \\ Germany. Fax: +4955139 3373; Tel: +4955139 33001; \\ E-mail: hroesky@gwdg.de; dstalke@chemie.uni-goettingen; koley@iiserkol.ac.in
}

\section{Content:}

(S1) Syntheses of compounds $2, \mathbf{4 , 6}$ and 8

(S2) Crystal data of 2, 4 and 6

(S3) Selected bond parameters of 2, 4 and $\mathbf{6}$

(S4) Theoretical calculation

(S5) References 
(S1) Syntheses of compounds 2, 4, 6 and 8:

All reactions and handlings of reagents were performed under an atmosphere of dry nitrogen using standard Schlenk techniques or a glove box where the $\mathrm{O}_{2}$ and $\mathrm{H}_{2} \mathrm{O}$ levels were usually kept below 1 ppm. cAAC ${ }^{\text {S1a }}$, $\mathrm{RSiHCl}_{2}\left(\mathrm{R}=\mathrm{Ar}-\left(\mathrm{SiMe}_{3}\right) \mathrm{N}, \mathrm{Ar}=2,6-i \mathrm{Pr}_{2} \mathrm{C}_{6} \mathrm{H}_{3}\right)(\mathbf{1})^{\mathrm{S} 1 \mathrm{~b}}, \mathrm{Cp}^{*} \mathrm{SiHCl}_{2}(\mathbf{3}){ }^{\mathrm{S} 1 \mathrm{c}, \mathrm{d}}$, and $\mathrm{LSiHCl}_{2}\left(\mathrm{~L}=\mathrm{PhC}(\mathrm{NtBu})_{2}\right)^{\mathrm{S} 1 \mathrm{e}}$ (5) were prepared according to similar literature methods. The $\mathrm{SiHCl}_{3}(\mathbf{7})$ was purchased from Sigma Aldrich and used as such. Solvents were purified with the M-Braun Solvent Purification System. Solution NMR spectra were recorded on Bruker Avance 200, Bruker Avance 300, and Bruker Avance 500 MHz NMR spectrometers. Deuterated NMR solvent THF-d ${ }^{8}$ was dried by stirring for 2 days over $\mathrm{Na} / \mathrm{K}$ alloy followed by distillation in vacuum and degassed. EI-MS spectrum was obtained with a Finnigan MAT 8230 or a Varian MAT CH5 instrument $(70 \mathrm{eV})$ by EI-MS methods. Elemental analysis was performed by the Analytisches Labor des Instituts für Anorganische Chemie der Universität Göttingen. Melting point was measured in a sealed glass tube on a Büchi B-540 melting point apparatus.

$\mathbf{R S i C l}_{\mathbf{2}}(\mathbf{c A A C H})(\mathbf{2})$ : A mixture of $\mathbf{1}(270 \mathrm{mg} ; 0.775 \mathrm{mmol})$ and cAAC (221 $\left.\mathrm{mg} ; 0.775 \mathrm{mmol}\right)$ was placed into a $100 \mathrm{~mL}$ round bottom flask and $50 \mathrm{~mL}$ of toluene was added to it at $-78{ }^{\circ} \mathrm{C}$. The reaction mixture was allowed to warm to room temperature and was then continued for overnight. The reaction mixture was filtered and the solvent was evaporated under high vacuum to give the colorless product $\mathbf{2}$ (74\% yield). Single crystals of 2 were grown from toluene solution at $-26{ }^{\circ} \mathrm{C}$ temperature. The X-ray single crystal analysis revealed the structure of 2. Elemental analysis found in \% (calcd) for $\mathrm{C}_{35} \mathrm{H}_{58} \mathrm{Cl}_{2} \mathrm{~N}_{2} \mathrm{Si}_{2}: \mathrm{C}, 66.72$ (66.31); H, 9.71 (9.22); $\mathrm{N}$, 4.37 (4.42). The compound melts at $176{ }^{\circ} \mathrm{C},{ }^{1} \mathrm{H}$ NMR (298 K, THF-d 8 , $\delta$ ppm, 500.133 MHz): 7.21-7.00 (m, 6H, $\left.H_{\mathrm{ar}}\right), 4.03\left(\mathrm{~m}, 1 \mathrm{H}, \mathrm{CHMe}\right.$ ), $3.89(\mathrm{~s}, 1 \mathrm{H}, \mathrm{cAACH}), 3.64\left(\mathrm{~m}, 1 \mathrm{H}, \mathrm{CHMe} \mathrm{M}_{2}\right), 3.47(\mathrm{~m}, 1 \mathrm{H}, \mathrm{CHMe}), 3.06(\mathrm{~m}$, $1 \mathrm{H}, \mathrm{CHMe}$ ), 2.31(s, 2H, CH $\left.\mathrm{CH}_{2}\right), 1.55$ (s, 3H, NCMe 2 ), 1.38-1.29 (m, 12H, CHMe $) 1.27\left(\mathrm{~s}, 3 \mathrm{H}, \mathrm{NCMe}_{2}\right), 1.23-$ 1.21 (d, 3H, CHMe $), 1.21-1.18$ (d, 3H, CHMe $), 1.17-1.15$ (d, 3H, CHMe $), 1.15-1.12$ (d, 3H, CHMe $), 0.94(\mathrm{~s}$, 3H, $\left.\mathrm{CM} e_{2}\right), 0.85$ (s, 3H, CMe 2$), 0.20$ (s, 9H, SiMe $)$. 
${ }^{13}$ C NMR (298 K, THF-d 8 , $\delta$ ppm, 126 MHz): 152.19, 148.86, 147.81, 147.50, 145.24, 141.54, 129.65, 128.88, $126.59,125.94,125.37,125.31,124.92,76.62,65.63,61.36,41.77,32.93,32.43,31.64,29.93,29.21,28.96$, $28.71,27.98,27.01,26.90,26.33,4.50$.

${ }^{29}$ Si NMR (298 K, THF-d $8, \delta$ ppm, $\left.99.38 \mathrm{MHz}\right): 10.42$ and -7.79.

EI-MS: $m / z(\%) 631.2(100)[\mathrm{M}-\mathrm{H}]^{+}, 632.2(43)[\mathrm{M}-\mathrm{H}]^{+}, 633.2(80)[\mathrm{M}-\mathrm{H}]^{+}, 634.2(34)[\mathrm{M}-\mathrm{H}]^{+}$.

Cp*Cl $\mathbf{l}_{2}(\mathbf{c A A C H})(\mathbf{4})$ : A mixture of $\mathbf{3}(200 \mathrm{mg} ; 0.851 \mathrm{mmol})$ and cAAC (374 mg; $\left.0.851 \mathrm{mmol}\right)$ was placed into a $100 \mathrm{~mL}$ round bottom flask and $50 \mathrm{~mL}$ of toluene was added as a solvent at $-78{ }^{\circ} \mathrm{C}$. The reaction mixture was allowed to warm to room temperature and stirring was continued for overnight. The reaction mixture was filtered and the solvent was evaporated under high vacuum to give the colorless product 4 ( $81 \%$ yield). Single crystals of $\mathbf{4}$ were grown from toluene solution at room temperature. The X-ray single crystal analysis revealed the structure of 4. Elemental analysis found in \% (calcd) for $\mathrm{C}_{30} \mathrm{H}_{47} \mathrm{Cl}_{2} \mathrm{NSi}$ : C, 69.20 (69.27); H, 9.10 (9.03); N, 2.69 (2.75). The compound melts at $187{ }^{\circ} \mathrm{C},{ }^{1} \mathrm{H}$ NMR (298 K, THF-d 8 , $\delta$ ppm, 500.133 MHz): 7.07-6.99 (m, 6H, $\left.H_{\mathrm{ar}}\right), 3.99(\mathrm{~m}, 1 \mathrm{H}, \mathrm{CHMe}), 3.48(\mathrm{~s}, 1 \mathrm{H}, \mathrm{cAACH}), 2.94(\mathrm{~m}, 1 \mathrm{H}, \mathrm{CHMe} 2), 2.07-2.04\left(\mathrm{~d}, 1 \mathrm{H}, \mathrm{CH}_{2}\right), 1.98(\mathrm{~s}$, $3 \mathrm{H}, \mathrm{Cp} * \mathrm{Me}), 1.84-1.81(\mathrm{~m}, 9 \mathrm{H}, \mathrm{Cp} * M e), 1.78-1.76\left(\mathrm{~d}, 1 \mathrm{H}, \mathrm{CH}_{2}\right), 1.54(\mathrm{~s}, 3 \mathrm{H}, \mathrm{Cp} * M e), 1.289-1.283(\mathrm{~m}, 6 \mathrm{H}$, $\mathrm{CHMe} 2$ ), 1.276 (s, 3H, NCMe 2 ), 1.24 (s, 3H, NCMe 2 ), 1.19-1.18 (d, 3H, CHMe $)$ ) 1.16-1.15 (d, 3H, CHMe $)$, 0.96 (s, 3H, CMe $), 0.84$ (s, 3H, CMe 2 ).

${ }^{13}$ C NMR (298 K, THF-d 8 , $\delta$ ppm, 126 MHz): 128.27, 127.51, 125.27, 124.64, 124.05, 123.44, 73.05, 64.45, $60.17,56.61,40.65,30.62,29.03,27.82,27.62,27.43,26.65,25.75,20.11,16.74,11.78,10.14$.

${ }^{29}$ Si NMR (298 K, THF-d $8, \delta$ ppm, $\left.99.38 \mathrm{MHz}\right): 24.72$. 


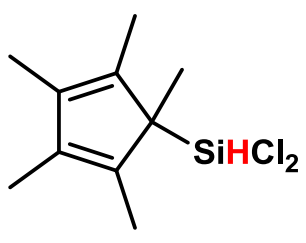

3

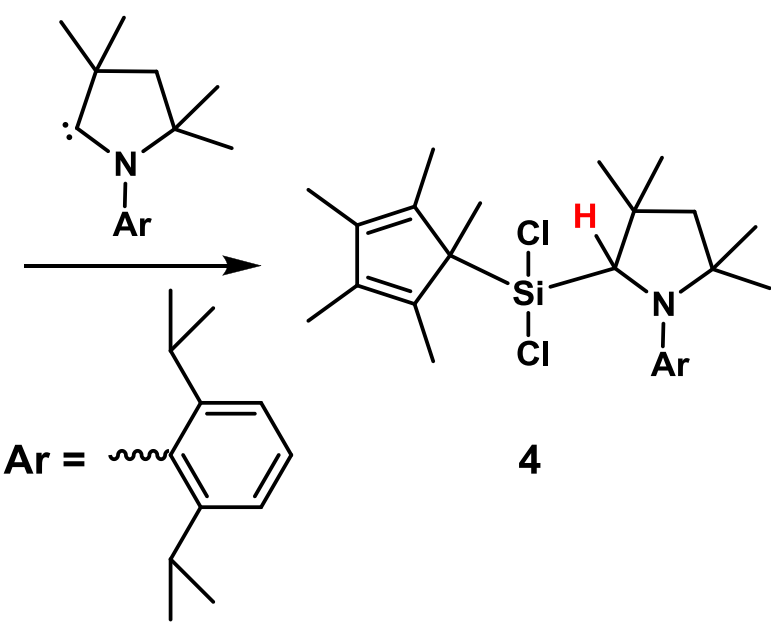

Scheme S1. Synthesis of compound 4.

$\mathbf{L S i C l}_{2}(\mathbf{c A A C H})(\mathbf{6})$ : A mixture of 5 (200 mg; $\left.0.603 \mathrm{mmol}\right)$ and cAAC (265 mg; $\left.0.603 \mathrm{mmol}\right)$ was placed into a $100 \mathrm{~mL}$ round bottom flask and $50 \mathrm{~mL}$ of toluene was added as a solvent at $-78{ }^{\circ} \mathrm{C}$. The reaction mixture was allowed to warm to room temperature and stirring was continued for three days to get a colorless solution. The reaction mixture was filtered and the solvent was evaporated under high vacuum to give the colorless product $\mathbf{6}(68 \%$ yield $)$. Single crystals of $\mathbf{6}$ were grown from toluene solution at room temperature. The X-ray single crystal analysis revealed the structure of 6 . Elemental analysis found in \% (calcd) for $\mathrm{C}_{35} \mathrm{H}_{55} \mathrm{Cl}_{2} \mathrm{~N}_{3} \mathrm{Si}$ : $\mathrm{C}, 68.15$ (68.21); H, 8.99 (8.87); N, 6.81 (6.92). The compound melts at $152{ }^{\circ} \mathrm{C},{ }^{1} \mathrm{H}$ NMR $\left(298 \mathrm{~K}, \mathrm{THF}-\mathrm{d}_{8}, \delta\right.$ ppm, $500.133 \mathrm{MHz}): 7.64-7.31\left(\mathrm{~m}, 6 \mathrm{H}, H_{\mathrm{ar}}\right), 4.15\left(\mathrm{~m}, 1 \mathrm{H}, \mathrm{CHMe} \mathrm{M}_{2}\right), 3.92(\mathrm{~s}, 1 \mathrm{H}, \mathrm{cAACH}), 3.11(\mathrm{~m}, 1 \mathrm{H}, \mathrm{CHMe} 2)$, 2.24-2.21 (d, 1H, $\left.\mathrm{CH}_{2}\right), 1.90-1.88\left(\mathrm{~d}, 1 \mathrm{H}, \mathrm{CH}_{2}\right), 1.64\left(\mathrm{~s}, 3 \mathrm{H}, \mathrm{NCMe} e_{2}\right), 1.52\left(\mathrm{~s}, 3 \mathrm{H}, \mathrm{NCMe} e_{2}\right), 1.36-1.35(\mathrm{~d}, 3 \mathrm{H}$, $\left.\mathrm{CHMe}_{2}\right), 1.30-1.29$ (d, 3H, CHMe $), 1.23-1.22$ (d, 3H, CHMe $), 1.19$ (s, 3H, CMe $), 1.18-1.16$ (d, 3H, CHMe $)$, 1.13-1.09 (m, 18H, N $\left.{ }^{t} B u\right), 0.92\left(\mathrm{~s}, 3 \mathrm{H}, \mathrm{CMe} e_{2}\right)$.

${ }^{13}$ C NMR (298 K, THF-d $8, \delta$ ppm, 126 MHz): 129.85, 129.63, 128.40, 126.56, 125.47, 124.73, 76.23, 61.40, 42.96, 34.32, 32.11, 32.06, 31.74, 31.64, 31.27, 31.16, 31.13, 29.50, 29.13, 27.98, 27.16.

${ }^{29}$ Si NMR (298 K, THF-d 8 , $\delta$ ppm, 99.38 MHz): -9.42. 


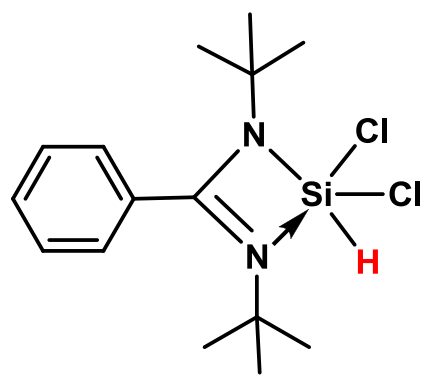

5

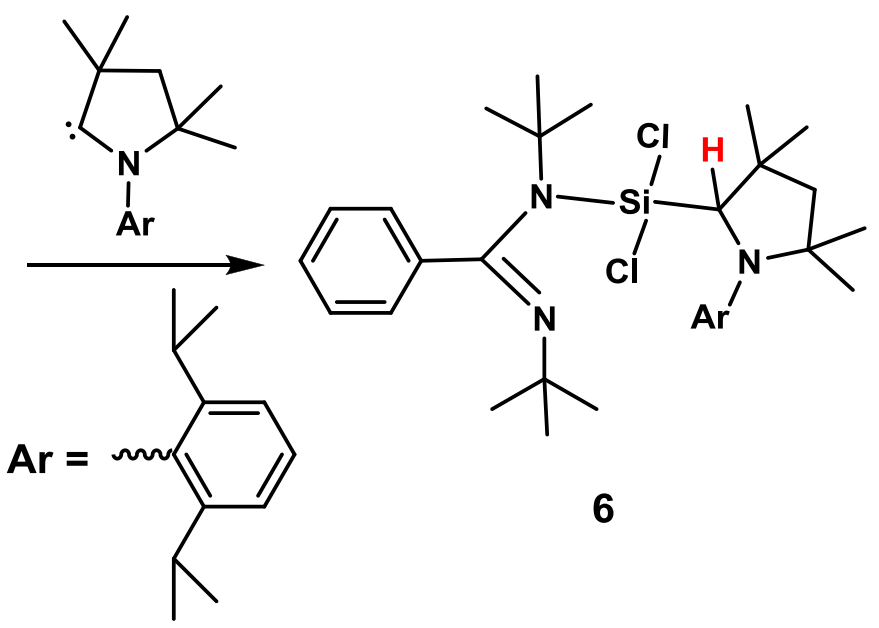

Scheme S2. Synthesis of compound 6 .

$\mathbf{S i C l}_{3}(\mathbf{c A A C H})(\mathbf{8})$ : The cAAC (200 $\left.\mathrm{mg} ; 0.452 \mathrm{mmol}\right)$ was placed into a $100 \mathrm{~mL}$ round bottom flask and $50 \mathrm{~mL}$ of toluene was added to it at room temperature. To this solution $\mathrm{SiHCl}_{3}(\mathbf{7})(0.045 \mathrm{~mL}, 0.452)$ was added drop by drop and then stirring was continued overnight. The reaction mixture was filtered and the solvent was evaporated under high vacuum to give the colorless oily product 8 (89\% yield). Elemental analysis found in \% (calcd) for $\mathrm{C}_{20} \mathrm{H}_{32} \mathrm{Cl}_{3} \mathrm{NSi}$ : C, 57.07 (57.12); H, 7.66 (7.58); N, 3.33 (3.41). ${ }^{1} \mathrm{H}$ NMR (298 K, THF-d,$\delta$ ppm, 500.133 MHz): 7.13-7.07 (m, 3H, $\left.H_{\mathrm{ar}}\right), 3.95(\mathrm{~m}, 1 \mathrm{H}, \mathrm{CHMe}$ ), $3.85(\mathrm{~s}, 1 \mathrm{H}, \mathrm{cAACH}), 3.02(\mathrm{~m}, 1 \mathrm{H}, \mathrm{CHMe}$ ), 2.25-2.23 (d, 1H, $\mathrm{CH}_{2}$ ), 1.99-1.96 (d, 1H, $\left.\mathrm{CH}_{2}\right), 1.67$ (s, 3H, $\mathrm{NCMe}_{2}$ ), 1.58 (s, 3H, $\left.\mathrm{NCMe} 2\right), 1.44$ (s, 3H, $\mathrm{CMe}_{2}$ ), 1.34-1.32 (d, 3H, CHMe 2 ), 1.27-1.25 (m, 6H, CHMe $)$ ), 1.18-1.17 (d, 3H, CHMe $), 0.94$ (s, 3H, $\mathrm{CMe}_{2}$ ).

${ }^{13}$ C NMR (298 K, THF-d $8, \delta$ ppm, 126 MHz): 127.58, 125.69, 125.00, 74.37, 60.03, 42.43, 32.86, 32.61, 29.58, $28.65,28.10,27.04$.

${ }^{29}$ Si NMR (298 K, THF-d $8, \delta$ ppm, 99.38 MHz): 3.85. 


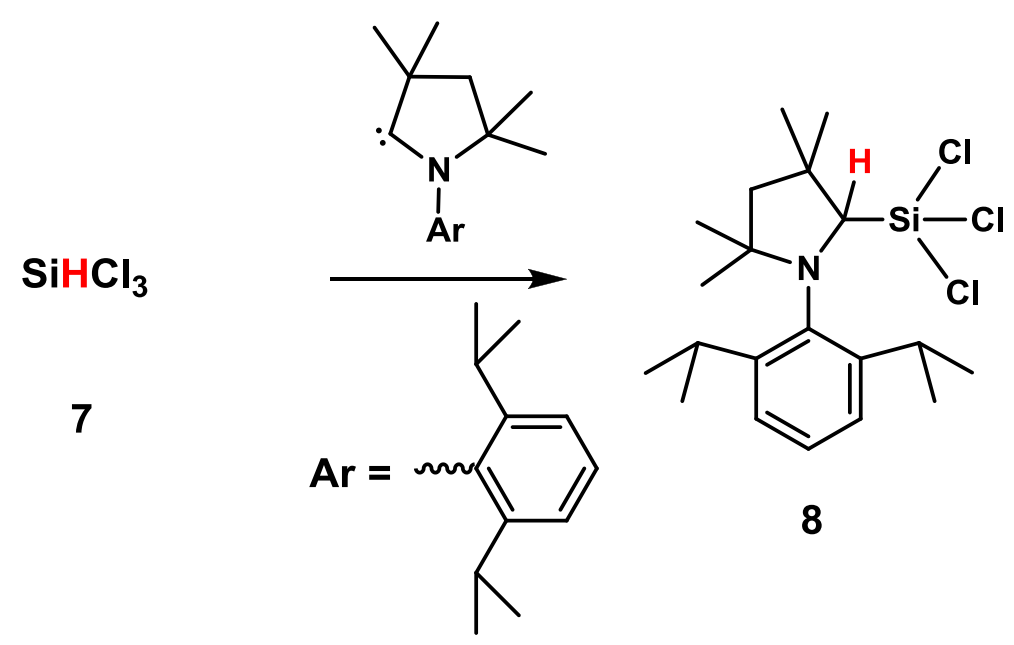

Scheme S3. Synthesis of compound 8 .
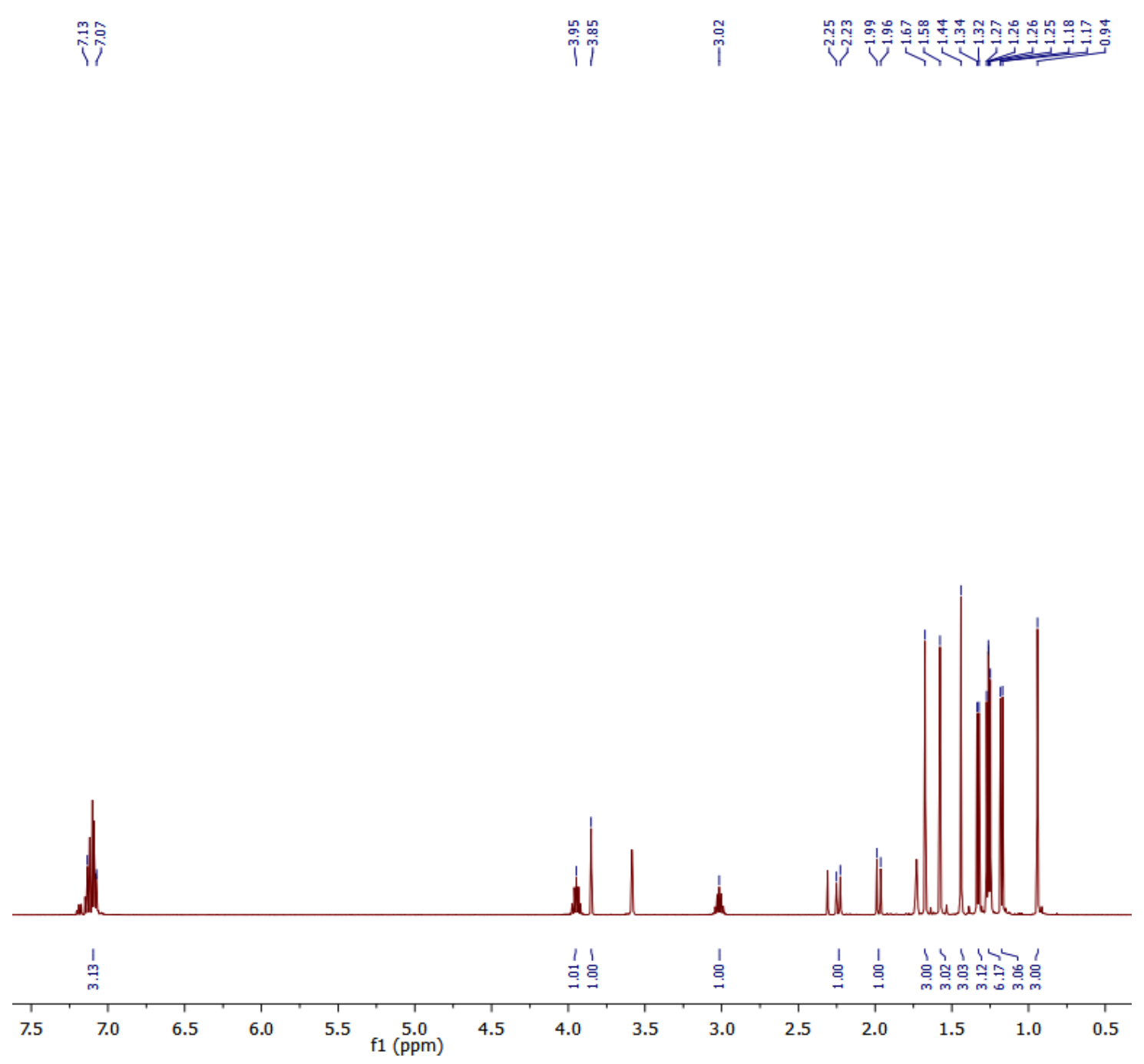

Figure S1. ${ }^{1} \mathrm{H}-\mathrm{NMR}$ spectrum of compound 8. 


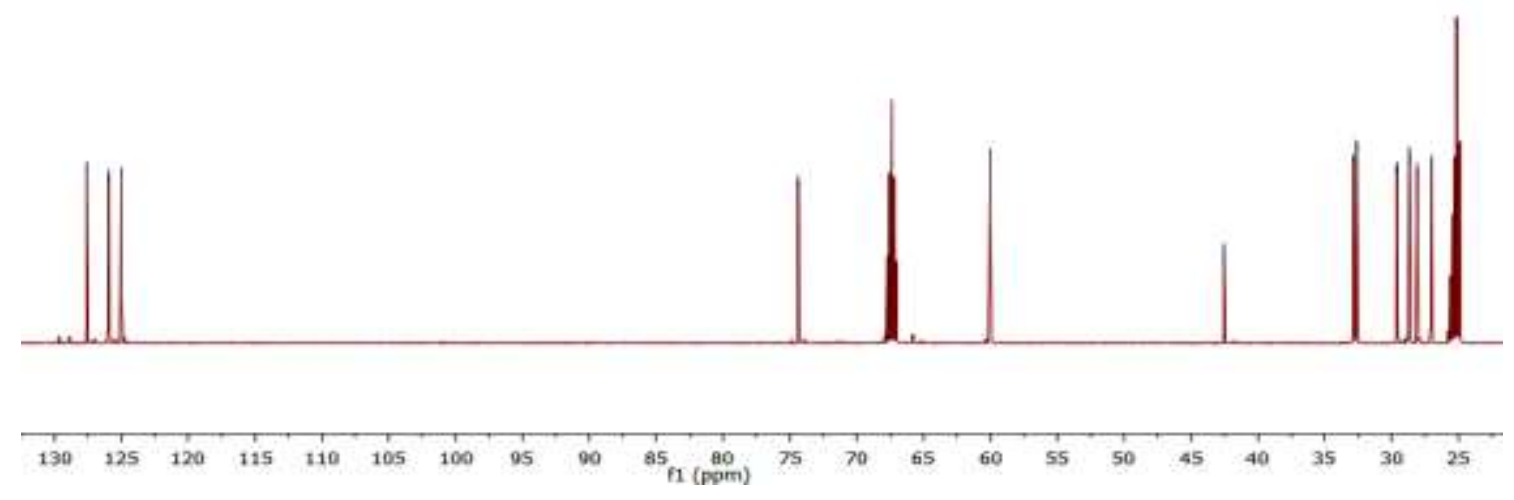

Figure S2. ${ }^{13} \mathrm{C}-\mathrm{NMR}$ spectrum of compound $\mathbf{8}$.

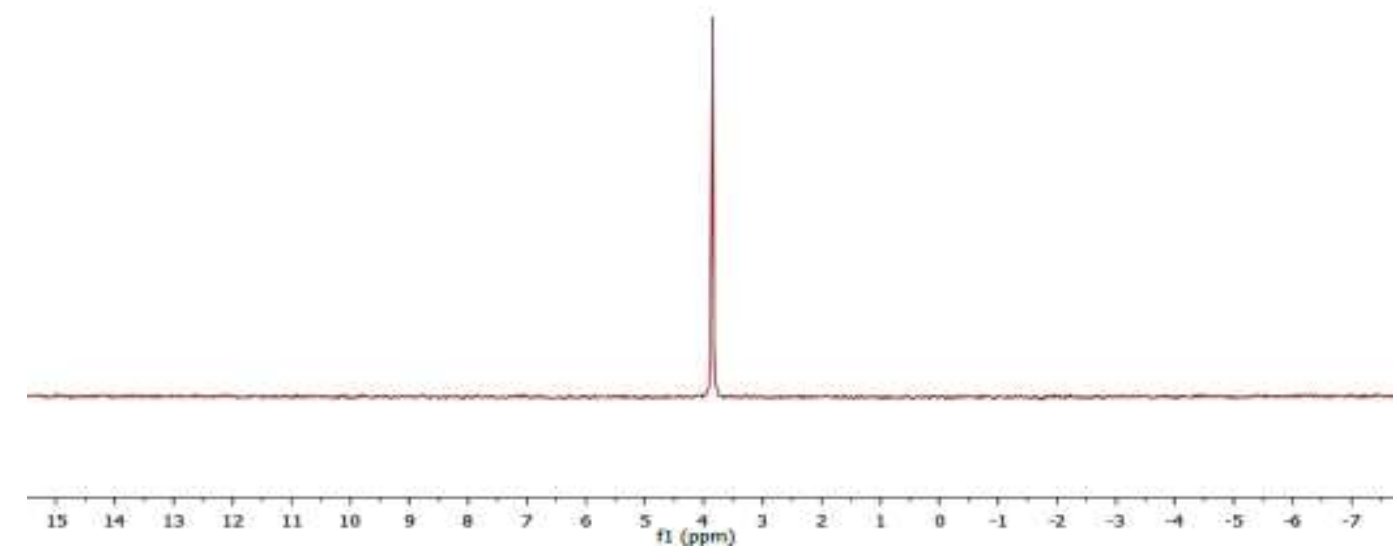

Figure S3. ${ }^{29} \mathrm{Si}$-NMR spectrum of compound $\mathbf{8}$. 


\section{(S2) Crystal data of 2, 4, and 6}

Crystal Structure Determination. Suitable single crystals for X-ray structural analysis of compound 2, 4, and 6 were mounted at low temperature in inert oil under argon atmosphere by applying the X-Temp2 device. ${ }^{\mathrm{S} 2}$ The data of compound 2 were collected on a Bruker D8 three circle diffractometer equipped with a SMART APEX II CCD detector and INCOATEC Mo microfocus source with INCOATEC Quazar mirror optics. ${ }^{\text {S3 }}$ The data of compounds 4 and $\mathbf{6}$ were collected on a Bruker D8 three circle diffractometer equipped with a SMART APEX II CCD detector and INCOATEC Ag microfocus source with INCOATEC Quazar mirror optics. ${ }^{\text {S3 }}$ All data were integrated with SAINT 8.30C. ${ }^{\mathrm{S} 4}$ Semi-empirical absorption corrections were applied with SADABS. ${ }^{\text {S5 }}$ The structures were solved by direct methods (SHELXT2014) ${ }^{\mathrm{S} 6}$ and refined against all data by full-matrix least-squares methods on $F^{2}$ (SHELXL-2014/7). ${ }^{\text {S7 }}$ All non-hydrogen-atoms were refined with anisotropic displacement parameters. All hydrogen atoms, except H1 in compound 2, H14 in compound 4 and H4 in compound 6 were refined on calculated positions using a riding model. Their $\mathrm{U}_{\text {iso }}$ values were constrained to $1.5 \mathrm{U}_{\mathrm{eq}}$ of their pivot atoms for terminal $\mathrm{sp}^{3}$ carbon atoms and 1.2 times for all other carbon atoms. The positions of these three hydrogen atomswere freely refined with $\mathrm{U}_{\text {iso }}$ values constrained to $1.2 \mathrm{U}_{\text {eq }}$ of its pivot atom.

Table S1. Crystal data and structure refinement for 2, 4, and 6:

\begin{tabular}{|c|c|c|c|}
\hline Compound & 2 & 4 & 6 \\
\hline Empirical formula & $\mathrm{C}_{38.50} \mathrm{H}_{62} \mathrm{Cl}_{2} \mathrm{~N}_{2} \mathrm{Si}_{2}$ & $\mathrm{C}_{30} \mathrm{H}_{47} \mathrm{C}_{12} \mathrm{~N} \mathrm{Si}$ & $\mathrm{C}_{35} \mathrm{H}_{55} \mathrm{Cl}_{2} \mathrm{~N}_{3} \mathrm{Si}$ \\
\hline CCDC no. & 1419453 & 1443738 & 1443739 \\
\hline Molecular weight & 679.98 & 520.67 & 616.81 \\
\hline Crystal size $[\mathrm{mm}]$ & $0.190 \times 0.110 \times 0.100$ & $0.404 \times 0.385 \times 0.245$ & $0.196 \times 0.170 \times 0.062$ \\
\hline Wavelength $[\AA]]$ & 0.71073 & 0.56086 & 0.56086 \\
\hline Crystal system & monoclinic & monoclinic & monoclinic \\
\hline Space group & $P 2_{1} / c$ & $P 2_{1}$ & $P 2_{1} / c$ \\
\hline$a[\AA]$ & $19.688(3)$ & $8.815(2)$ & $13.314(2)$ \\
\hline$b[\AA]$ & $11.024(2)$ & $16.427(3)$ & $16.570(2)$ \\
\hline$c[\AA]$ & $20.330(3)$ & $10.972(2)$ & $17.340(3)$ \\
\hline$\beta\left[^{\circ}\right]$ & $118.24(2)^{\circ}$ & $113.64(2)$ & $112.38(2)$ \\
\hline$V\left[\AA^{3}\right]$ & $3887.2(13)$ & $1455.5(5)$ & $3537.3(10)$ \\
\hline Z & 4 & 2 & 4 \\
\hline Temperature $[\mathrm{K}]$ & $100(2)$ & $100(2)$ & $100(2)$ \\
\hline$\rho\left[\mathrm{Mgm}^{-3}\right]$ & 1.162 & 1.188 & 1.158 \\
\hline$\mu\left[\mathrm{mm}^{-1}\right]$ & 0.257 & 0.151 & 0.132 \\
\hline$F(000)$ & 1476 & 564 & 1336 \\
\hline$\theta$-area $\left[^{\circ}\right]$ & 1.174 to $28.695^{\circ}$. & 1.599 to 23.694 & 1.305 to 19.510 \\
\hline Total number reflect. & 84942 & 60081 & 104005 \\
\hline Unique reflections & 10040 & 8956 & 6257 \\
\hline$R_{\text {int }}$ & 0.0340 & 0.0385 & 0.0842 \\
\hline Number of restraints & 50 & 1 & 0 \\
\hline Parameters & 453 & 323 & 387 \\
\hline$R 1[I>2 \sigma(I)]$ & 0.0304 & 0.0263 & 0.0355 \\
\hline$w R 2[I>2 \sigma(I)]$ & 0.0784 & 0.0617 & 0.0776 \\
\hline$R 1$ [all data] & 0.0364 & 0.0293 & 0.0504 \\
\hline$w R 2$ [all data] & 0.0829 & 0.0633 & 0.0841 \\
\hline GooF & 1.035 & 1.025 & 1.030 \\
\hline Absolute structure parameter & & $-0.03(2)$ & \\
\hline $\begin{array}{l}\text { Largest diff. peak / hole } \\
\text { max. / min. }\left[\cdot e \cdot \AA^{-3}\right]\end{array}$ & 0.419 and -0.209 & 0.244 and -0.169 & 0.381 and -0.319 \\
\hline
\end{tabular}



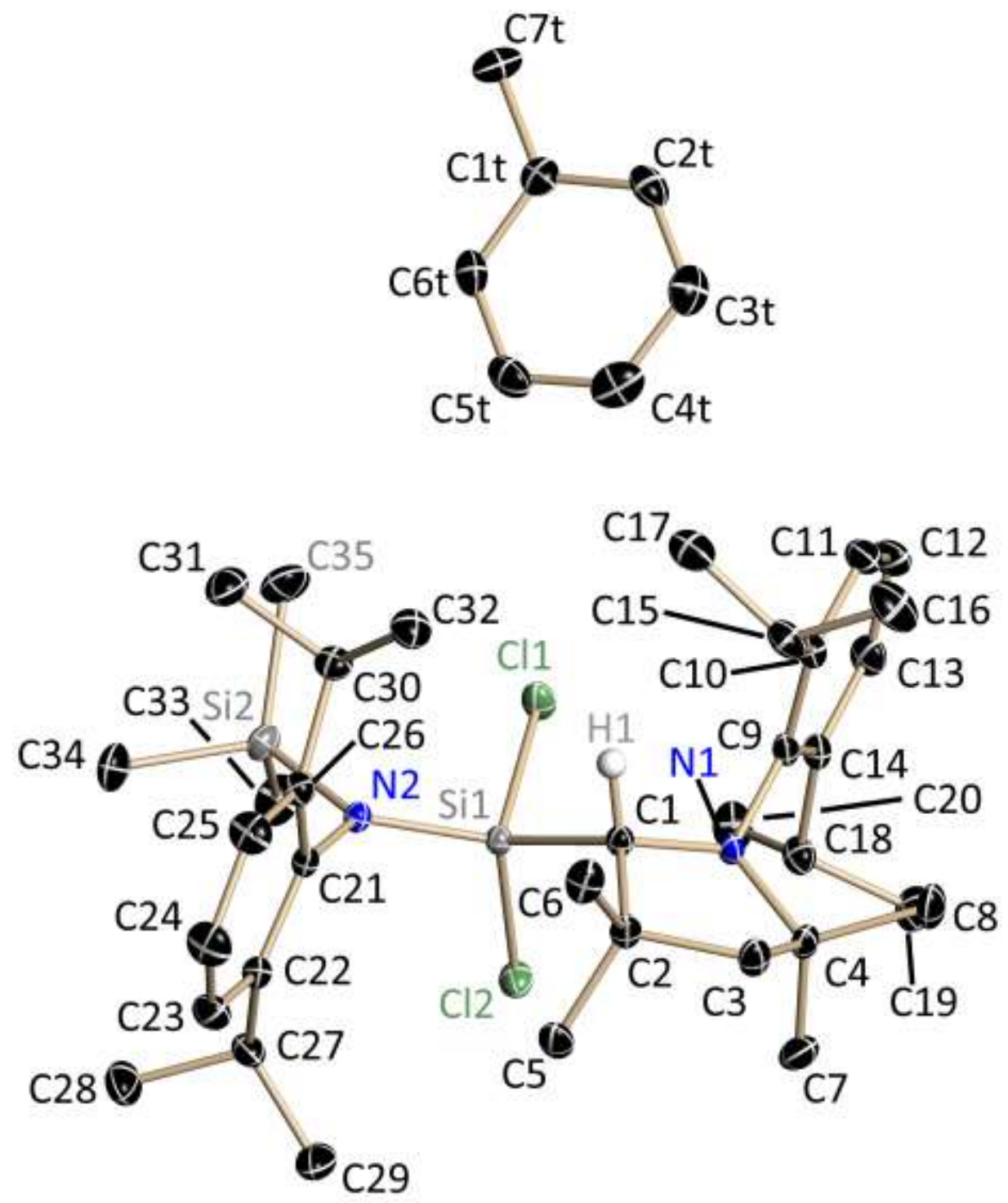

Figure S4 Molecular structure of compound 2. H atoms, except H1, and solvent molecule are omitted for clarity. Anisotropic displacement parameters are depicted at the $50 \%$ probability level.

The toluene molecule was refined on two equally occupied positions ignoring the inversion center ("PART -1").. For the 1,2- and 1,3 distances "SADI"-restraints were applied. The atomic displacement parameters were refined using "SIMU" and "RIGU". The position of the hydrogen atom $\mathrm{H} 1$ was refined freely. 


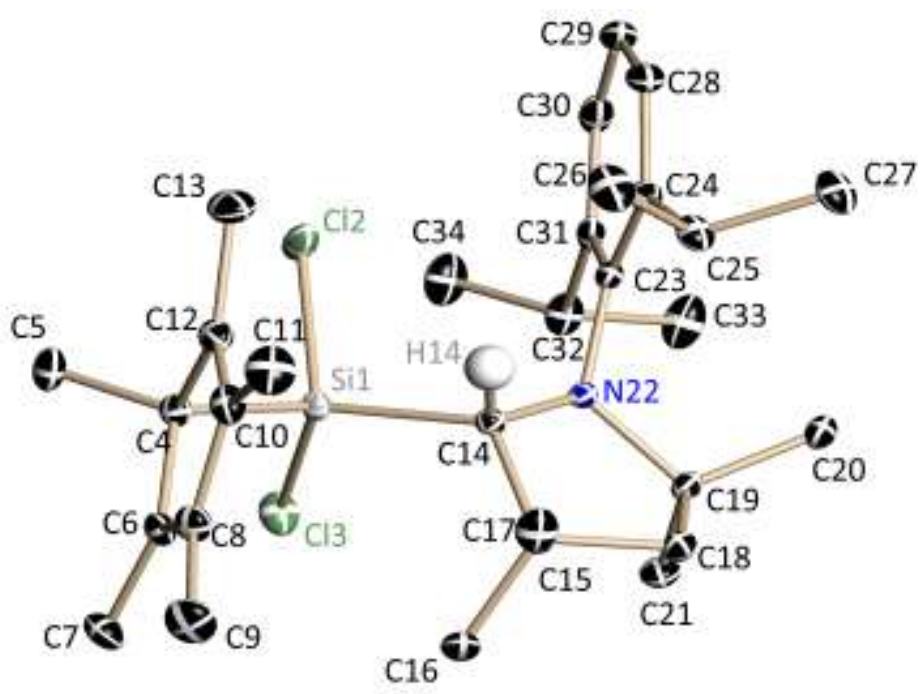

Figure S5 Molecular structure of compound 4. H atoms except H14 are omitted for clarity. Anisotropic displacement parameters are depicted at the $50 \%$ probability level.

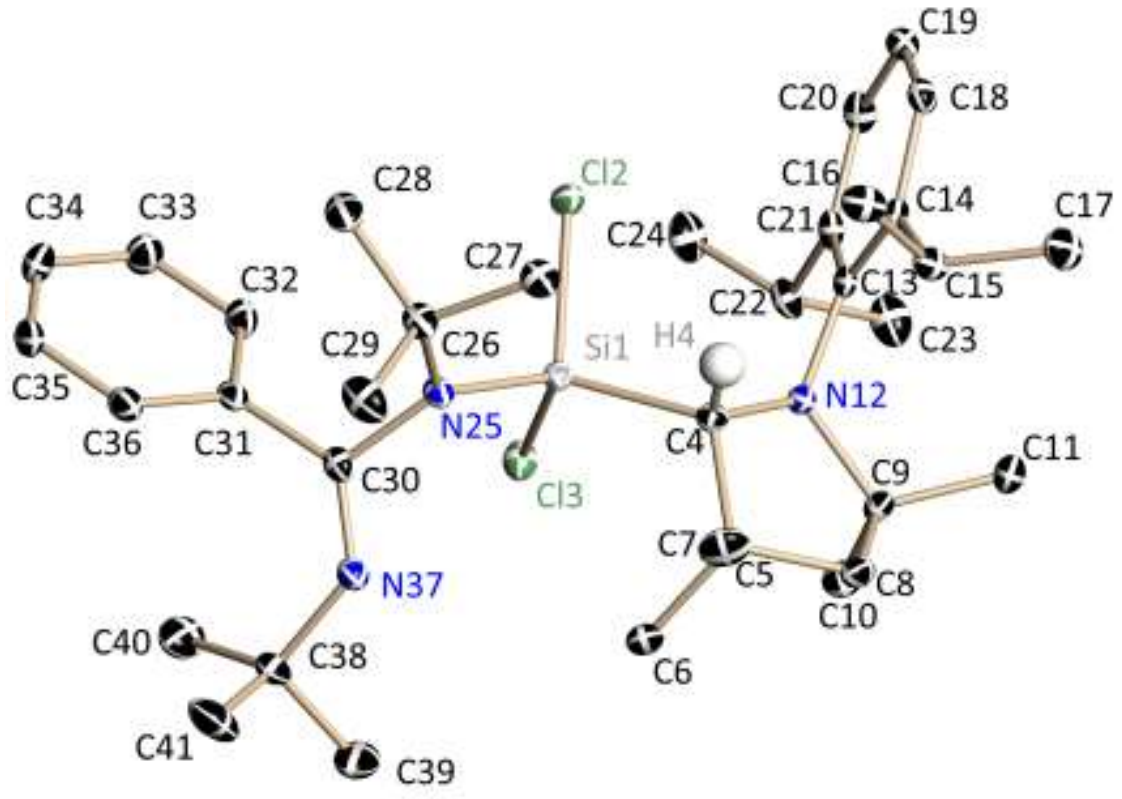

Figure S6 Molecular structure of compound 6. H atoms except $\mathrm{H} 4$ are omitted for clarity. Anisotropic displacement parameters are depicted at the $50 \%$ probability level. 


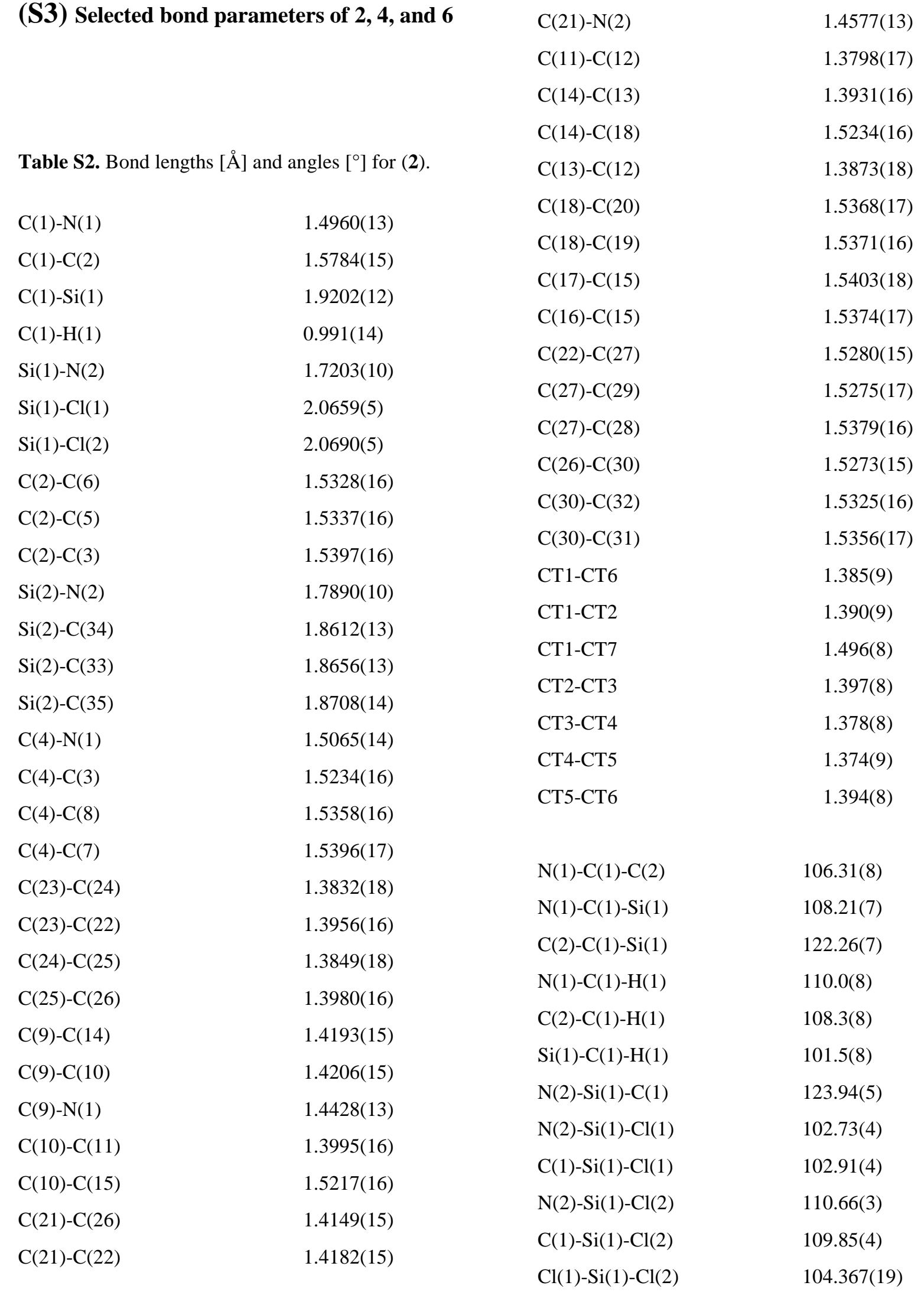

Table S2. Bond lengths $[\AA]$ and angles $\left[^{\circ}\right]$ for (2). 


\begin{tabular}{|c|c|c|c|}
\hline$C(6)-C(2)-C(5)$ & 107.24(9) & $C(12)-C(13)-C(14)$ & $121.54(11)$ \\
\hline$C(6)-C(2)-C(3)$ & 109.29(9) & $\mathrm{C}(14)-\mathrm{C}(18)-\mathrm{C}(20)$ & $112.92(10)$ \\
\hline $\mathrm{C}(5)-\mathrm{C}(2)-\mathrm{C}(3)$ & $111.15(9)$ & $\mathrm{C}(14)-\mathrm{C}(18)-\mathrm{C}(19)$ & $110.75(9)$ \\
\hline$C(6)-C(2)-C(1)$ & $112.18(9)$ & $\mathrm{C}(20)-\mathrm{C}(18)-\mathrm{C}(19)$ & $108.46(10)$ \\
\hline $\mathrm{C}(5)-\mathrm{C}(2)-\mathrm{C}(1)$ & $114.42(9)$ & $C(10)-C(15)-C(16)$ & $112.51(10)$ \\
\hline$C(3)-C(2)-C(1)$ & $102.50(8)$ & $\mathrm{C}(10)-\mathrm{C}(15)-\mathrm{C}(17)$ & $110.46(10)$ \\
\hline$N(2)-\operatorname{Si}(2)-C(34)$ & $108.39(5)$ & $C(16)-C(15)-C(17)$ & $109.39(11)$ \\
\hline $\mathrm{N}(2)-\operatorname{Si}(2)-\mathrm{C}(33)$ & $110.36(5)$ & $C(23)-C(22)-C(21)$ & $118.37(10)$ \\
\hline $\mathrm{C}(34)-\mathrm{Si}(2)-\mathrm{C}(33)$ & $107.92(6)$ & $\mathrm{C}(23)-\mathrm{C}(22)-\mathrm{C}(27)$ & $117.96(10)$ \\
\hline$N(2)-\operatorname{Si}(2)-C(35)$ & $114.40(5)$ & $\mathrm{C}(21)-\mathrm{C}(22)-\mathrm{C}(27)$ & $123.63(10)$ \\
\hline $\mathrm{C}(34)-\mathrm{Si}(2)-\mathrm{C}(35)$ & $106.72(6)$ & $\mathrm{C}(11)-\mathrm{C}(12)-\mathrm{C}(13)$ & $119.16(11)$ \\
\hline $\mathrm{C}(33)-\mathrm{Si}(2)-\mathrm{C}(35)$ & $108.82(7)$ & $\mathrm{C}(29)-\mathrm{C}(27)-\mathrm{C}(22)$ & $113.30(10)$ \\
\hline $\mathrm{N}(1)-\mathrm{C}(4)-\mathrm{C}(3)$ & $102.97(9)$ & $\mathrm{C}(29)-\mathrm{C}(27)-\mathrm{C}(28)$ & $107.34(10)$ \\
\hline $\mathrm{N}(1)-\mathrm{C}(4)-\mathrm{C}(8)$ & $112.32(9)$ & $\mathrm{C}(22)-\mathrm{C}(27)-\mathrm{C}(28)$ & $111.53(9)$ \\
\hline$C(3)-C(4)-C(8)$ & $110.29(9)$ & $C(25)-C(26)-C(21)$ & $118.52(10)$ \\
\hline $\mathrm{N}(1)-\mathrm{C}(4)-\mathrm{C}(7)$ & $111.53(9)$ & $C(25)-C(26)-C(30)$ & $118.08(10)$ \\
\hline$C(3)-C(4)-C(7)$ & $111.74(10)$ & $C(21)-C(26)-C(30)$ & $123.27(10)$ \\
\hline C(8)-C(4)-C(7) & 107.99(10) & $C(26)-C(30)-C(32)$ & $113.57(10)$ \\
\hline $\mathrm{C}(4)-\mathrm{C}(3)-\mathrm{C}(2)$ & $107.73(9)$ & $\mathrm{C}(26)-\mathrm{C}(30)-\mathrm{C}(31)$ & $110.05(10)$ \\
\hline $\mathrm{C}(24)-\mathrm{C}(23)-\mathrm{C}(22)$ & $121.64(11)$ & $\mathrm{C}(32)-\mathrm{C}(30)-\mathrm{C}(31)$ & $108.36(10)$ \\
\hline$C(23)-C(24)-C(25)$ & $119.57(11)$ & $\mathrm{C}(21)-\mathrm{N}(2)-\mathrm{Si}(1)$ & $122.20(7)$ \\
\hline$C(24)-C(25)-C(26)$ & $121.42(11)$ & $\mathrm{C}(21)-\mathrm{N}(2)-\mathrm{Si}(2)$ & $115.81(7)$ \\
\hline $\mathrm{C}(14)-\mathrm{C}(9)-\mathrm{C}(10)$ & $118.84(10)$ & $\mathrm{Si}(1)-\mathrm{N}(2)-\mathrm{Si}(2)$ & $121.94(5)$ \\
\hline $\mathrm{C}(14)-\mathrm{C}(9)-\mathrm{N}(1)$ & 118.62(9) & $\mathrm{C}(9)-\mathrm{N}(1)-\mathrm{C}(1)$ & 121.41(9) \\
\hline $\mathrm{C}(10)-\mathrm{C}(9)-\mathrm{N}(1)$ & $122.51(10)$ & $\mathrm{C}(9)-\mathrm{N}(1)-\mathrm{C}(4)$ & $118.98(8)$ \\
\hline $\mathrm{C}(11)-\mathrm{C}(10)-\mathrm{C}(9)$ & $119.20(10)$ & $\mathrm{C}(1)-\mathrm{N}(1)-\mathrm{C}(4)$ & $110.67(8)$ \\
\hline $\mathrm{C}(11)-\mathrm{C}(10)-\mathrm{C}(15)$ & $116.81(10)$ & CT6-CT1-CT2 & $118.2(5)$ \\
\hline C(9)-C(10)-C(15) & $123.99(10)$ & CT6-CT1-CT7 & $120.8(5)$ \\
\hline$C(26)-C(21)-C(22)$ & $120.27(10)$ & CT2-CT1-CT7 & $121.0(5)$ \\
\hline $\mathrm{C}(26)-\mathrm{C}(21)-\mathrm{N}(2)$ & $118.91(9)$ & CT1-CT2-CT3 & $121.1(6)$ \\
\hline $\mathrm{C}(22)-\mathrm{C}(21)-\mathrm{N}(2)$ & $120.74(9)$ & CT4-CT3-СТ2 & $119.4(5)$ \\
\hline $\mathrm{C}(12)-\mathrm{C}(11)-\mathrm{C}(10)$ & $121.70(11)$ & СТ5-СТ4-СТ3 & $120.4(6)$ \\
\hline C(13)-C(14)-C(9) & $119.50(10)$ & СТ4-СТ5-СТ6 & $119.9(5)$ \\
\hline $\mathrm{C}(13)-\mathrm{C}(14)-\mathrm{C}(18)$ & $117.34(10)$ & СТ1-СТ6-СТ5 & 121.1(6) \\
\hline $\mathrm{C}(9)-\mathrm{C}(14)-\mathrm{C}(18)$ & $123.15(10)$ & & \\
\hline
\end{tabular}


Table S3. Bond lengths $[\AA]$ and angles $\left[{ }^{\circ}\right]$ for (4).

\begin{tabular}{|c|c|}
\hline $\operatorname{Si}(1)-C(14)$ & $1.9088(16)$ \\
\hline $\operatorname{Si}(1)-C(4)$ & $1.9119(16)$ \\
\hline $\mathrm{Si}(1)-\mathrm{Cl}(2)$ & $2.0682(6)$ \\
\hline $\mathrm{Si}(1)-\mathrm{Cl}(3)$ & $2.0685(6)$ \\
\hline$C(4)-C(6)$ & $1.510(2)$ \\
\hline $\mathrm{C}(4)-\mathrm{C}(12)$ & $1.521(2)$ \\
\hline$C(4)-C(5)$ & $1.544(2)$ \\
\hline$C(6)-C(8)$ & $1.350(2)$ \\
\hline$C(6)-C(7)$ & $1.499(2)$ \\
\hline $\mathrm{C}(8)-\mathrm{C}(10)$ & $1.465(2)$ \\
\hline $\mathrm{C}(8)-\mathrm{C}(9)$ & $1.502(2)$ \\
\hline$C(10)-C(12)$ & $1.356(2)$ \\
\hline$C(10)-C(11)$ & $1.503(2)$ \\
\hline $\mathrm{C}(12)-\mathrm{C}(13)$ & $1.498(2)$ \\
\hline $\mathrm{C}(14)-\mathrm{N}(22)$ & $1.4877(19)$ \\
\hline$C(14)-C(15)$ & $1.575(2)$ \\
\hline $\mathrm{C}(14)-\mathrm{H}(14)$ & $0.99(2)$ \\
\hline $\mathrm{C}(15)-\mathrm{C}(16)$ & $1.533(2)$ \\
\hline$C(15)-C(17)$ & $1.534(2)$ \\
\hline$C(15)-C(18)$ & $1.542(2)$ \\
\hline$C(18)-C(19)$ & $1.531(2)$ \\
\hline $\mathrm{C}(19)-\mathrm{N}(22)$ & $1.499(2)$ \\
\hline C(19)-C(20) & $1.529(2)$ \\
\hline$C(19)-C(21)$ & $1.545(2)$ \\
\hline $\mathrm{N}(22)-\mathrm{C}(23)$ & $1.4370(19)$ \\
\hline $\mathrm{C}(23)-\mathrm{C}(31)$ & $1.414(2)$ \\
\hline $\mathrm{C}(23)-\mathrm{C}(24)$ & $1.420(2)$ \\
\hline$C(24)-C(28)$ & $1.394(2)$ \\
\hline$C(24)-C(25)$ & $1.526(2)$ \\
\hline$C(25)-C(26)$ & $1.536(2)$ \\
\hline $\mathrm{C}(25)-\mathrm{C}(27)$ & $1.539(2)$ \\
\hline$C(28)-C(29)$ & $1.380(3)$ \\
\hline$C(29)-C(30)$ & $1.386(3)$ \\
\hline$C(30)-C(31)$ & $1.398(2)$ \\
\hline C(31)-C(32) & $1.514(2)$ \\
\hline $\mathrm{C}(32)-\mathrm{C}(34)$ & $1.533(2)$ \\
\hline
\end{tabular}

\begin{tabular}{|c|c|}
\hline$C(14)-\operatorname{Si}(1)-C(4)$ & $119.99(7)$ \\
\hline $\mathrm{C}(14)-\mathrm{Si}(1)-\mathrm{Cl}(2)$ & $106.91(5)$ \\
\hline $\mathrm{C}(4)-\mathrm{Si}(1)-\mathrm{Cl}(2)$ & $103.23(5)$ \\
\hline $\mathrm{C}(14)-\mathrm{Si}(1)-\mathrm{Cl}(3)$ & $111.59(5)$ \\
\hline $\mathrm{C}(4)-\mathrm{Si}(1)-\mathrm{Cl}(3)$ & $110.62(5)$ \\
\hline $\mathrm{Cl}(2)-\mathrm{Si}(1)-\mathrm{Cl}(3)$ & $102.57(3)$ \\
\hline $\mathrm{C}(6)-\mathrm{C}(4)-\mathrm{C}(12)$ & $102.83(12)$ \\
\hline$C(6)-C(4)-C(5)$ & $111.79(13)$ \\
\hline$C(12)-C(4)-C(5)$ & $113.49(13)$ \\
\hline $\mathrm{C}(6)-\mathrm{C}(4)-\mathrm{Si}(1)$ & $111.83(11)$ \\
\hline$C(12)-C(4)-S i(1)$ & $106.63(10)$ \\
\hline $\mathrm{C}(5)-\mathrm{C}(4)-\mathrm{Si}(1)$ & $110.03(10)$ \\
\hline$C(8)-C(6)-C(7)$ & $127.10(15)$ \\
\hline $\mathrm{C}(8)-\mathrm{C}(6)-\mathrm{C}(4)$ & $109.25(14)$ \\
\hline$C(7)-C(6)-C(4)$ & $123.43(14)$ \\
\hline $\mathrm{C}(6)-\mathrm{C}(8)-\mathrm{C}(10)$ & $109.65(14)$ \\
\hline$C(6)-C(8)-C(9)$ & $127.31(16)$ \\
\hline $\mathrm{C}(10)-\mathrm{C}(8)-\mathrm{C}(9)$ & $122.99(15)$ \\
\hline$C(12)-C(10)-C(8)$ & $109.38(14)$ \\
\hline$C(12)-C(10)-C(11)$ & $128.07(17)$ \\
\hline $\mathrm{C}(8)-\mathrm{C}(10)-\mathrm{C}(11)$ & $122.55(16)$ \\
\hline $\mathrm{C}(10)-\mathrm{C}(12)-\mathrm{C}(13)$ & $126.92(15)$ \\
\hline $\mathrm{C}(10)-\mathrm{C}(12)-\mathrm{C}(4)$ & $108.79(14)$ \\
\hline $\mathrm{C}(13)-\mathrm{C}(12)-\mathrm{C}(4)$ & $124.22(15)$ \\
\hline $\mathrm{N}(22)-\mathrm{C}(14)-\mathrm{C}(15)$ & $106.73(12)$ \\
\hline $\mathrm{N}(22)-\mathrm{C}(14)-\mathrm{Si}(1)$ & $110.03(10)$ \\
\hline $\mathrm{C}(15)-\mathrm{C}(14)-\mathrm{Si}(1)$ & $120.72(10)$ \\
\hline $\mathrm{N}(22)-\mathrm{C}(14)-\mathrm{H}(14)$ & 109.1(11) \\
\hline $\mathrm{C}(15)-\mathrm{C}(14)-\mathrm{H}(14)$ & $108.8(12)$ \\
\hline $\mathrm{Si}(1)-\mathrm{C}(14)-\mathrm{H}(14)$ & $101.0(12)$ \\
\hline$C(16)-C(15)-C(17)$ & $107.87(13)$ \\
\hline$C(16)-C(15)-C(18)$ & $110.98(13)$ \\
\hline $\mathrm{C}(17)-\mathrm{C}(15)-\mathrm{C}(18)$ & $109.72(14)$ \\
\hline$C(16)-C(15)-C(14)$ & $114.94(13)$ \\
\hline$C(17)-C(15)-C(14)$ & $111.14(13)$ \\
\hline$C(18)-C(15)-C(14)$ & $102.08(12)$ \\
\hline
\end{tabular}




\begin{tabular}{|c|c|c|c|}
\hline C(19)-C(18)-C(15) & $107.87(13)$ & $\mathrm{C}(4)-\mathrm{H}(4)$ & $1.005(19)$ \\
\hline $\mathrm{N}(22)-\mathrm{C}(19)-\mathrm{C}(20)$ & $111.94(12)$ & $C(5)-C(8)$ & $1.536(3)$ \\
\hline $\mathrm{N}(22)-\mathrm{C}(19)-\mathrm{C}(18)$ & $103.04(12)$ & $C(5)-C(7)$ & $1.538(3)$ \\
\hline $\mathrm{C}(20)-\mathrm{C}(19)-\mathrm{C}(18)$ & 110.91(13) & $\mathrm{C}(5)-\mathrm{C}(6)$ & $1.540(3)$ \\
\hline $\mathrm{N}(22)-\mathrm{C}(19)-\mathrm{C}(21)$ & $111.65(12)$ & $\mathrm{C}(8)-\mathrm{C}(9)$ & $1.530(3)$ \\
\hline$C(20)-C(19)-C(21)$ & $108.09(13)$ & $\mathrm{C}(9)-\mathrm{N}(12)$ & $1.502(2)$ \\
\hline$C(18)-C(19)-C(21)$ & $111.22(13)$ & $C(9)-C(11)$ & $1.531(3)$ \\
\hline $\mathrm{C}(23)-\mathrm{N}(22)-\mathrm{C}(14)$ & $117.36(12)$ & $\mathrm{C}(9)-\mathrm{C}(10)$ & $1.542(3)$ \\
\hline $\mathrm{C}(23)-\mathrm{N}(22)-\mathrm{C}(19)$ & $121.58(12)$ & $\mathrm{N}(12)-\mathrm{C}(13)$ & $1.440(2)$ \\
\hline $\mathrm{C}(14)-\mathrm{N}(22)-\mathrm{C}(19)$ & $111.14(11)$ & $\mathrm{C}(13)-\mathrm{C}(21)$ & $1.415(3)$ \\
\hline $\mathrm{C}(31)-\mathrm{C}(23)-\mathrm{C}(24)$ & $119.17(14)$ & $\mathrm{C}(13)-\mathrm{C}(14)$ & $1.416(3)$ \\
\hline $\mathrm{C}(31)-\mathrm{C}(23)-\mathrm{N}(22)$ & $118.52(13)$ & $\mathrm{C}(14)-\mathrm{C}(18)$ & $1.396(3)$ \\
\hline $\mathrm{C}(24)-\mathrm{C}(23)-\mathrm{N}(22)$ & $122.20(13)$ & $C(14)-C(15)$ & $1.529(2)$ \\
\hline $\mathrm{C}(28)-\mathrm{C}(24)-\mathrm{C}(23)$ & $118.54(15)$ & $C(15)-C(17)$ & $1.533(3)$ \\
\hline$C(28)-C(24)-C(25)$ & $117.54(15)$ & $C(15)-C(16)$ & $1.533(3)$ \\
\hline $\mathrm{C}(23)-\mathrm{C}(24)-\mathrm{C}(25)$ & $123.77(14)$ & $\mathrm{C}(18)-\mathrm{C}(19)$ & $1.376(3)$ \\
\hline$C(24)-C(25)-C(26)$ & $113.64(15)$ & $C(19)-C(20)$ & $1.382(3)$ \\
\hline $\mathrm{C}(24)-\mathrm{C}(25)-\mathrm{C}(27)$ & $110.40(14)$ & $C(20)-C(21)$ & $1.389(3)$ \\
\hline$C(26)-C(25)-C(27)$ & 109.03(13) & $C(21)-C(22)$ & $1.523(3)$ \\
\hline$C(29)-C(28)-C(24)$ & $122.06(16)$ & $C(22)-C(24)$ & $1.533(3)$ \\
\hline$C(28)-C(29)-C(30)$ & $119.17(15)$ & $C(22)-C(23)$ & $1.538(3)$ \\
\hline $\mathrm{C}(29)-\mathrm{C}(30)-\mathrm{C}(31)$ & $121.06(16)$ & $\mathrm{N}(25)-\mathrm{C}(30)$ & $1.458(2)$ \\
\hline $\mathrm{C}(30)-\mathrm{C}(31)-\mathrm{C}(23)$ & $119.42(15)$ & $\mathrm{N}(25)-\mathrm{C}(26)$ & $1.517(2)$ \\
\hline $\mathrm{C}(30)-\mathrm{C}(31)-\mathrm{C}(32)$ & $118.55(14)$ & $C(26)-C(27)$ & $1.526(3)$ \\
\hline $\mathrm{C}(23)-\mathrm{C}(31)-\mathrm{C}(32)$ & $122.03(14)$ & $C(26)-C(29)$ & $1.527(3)$ \\
\hline $\mathrm{C}(31)-\mathrm{C}(32)-\mathrm{C}(34)$ & $113.64(14)$ & $C(26)-C(28)$ & $1.530(3)$ \\
\hline $\mathrm{C}(31)-\mathrm{C}(32)-\mathrm{C}(33)$ & $110.56(14)$ & $\mathrm{C}(30)-\mathrm{N}(37)$ & $1.261(2)$ \\
\hline $\mathrm{C}(34)-\mathrm{C}(32)-\mathrm{C}(33)$ & $109.00(14)$ & $\mathrm{C}(30)-\mathrm{C}(31)$ & $1.505(3)$ \\
\hline & & $\mathrm{C}(31)-\mathrm{C}(32)$ & $1.393(3)$ \\
\hline \multirow{2}{*}{\multicolumn{2}{|c|}{ Table S4. Bond lengths $[\AA ̊]$ and angles $\left[{ }^{\circ}\right]$ for $(\mathbf{6})$. }} & $\mathrm{C}(31)-\mathrm{C}(36)$ & $1.395(3)$ \\
\hline & & $C(32)-C(33)$ & $1.386(3)$ \\
\hline $\mathrm{Si}(1)-\mathrm{N}(25)$ & $1.7166(16)$ & $\mathrm{C}(33)-\mathrm{C}(34)$ & $1.386(3)$ \\
\hline $\mathrm{Si}(1)-\mathrm{C}(4)$ & $1.9048(18)$ & $\mathrm{C}(34)-\mathrm{C}(35)$ & $1.381(3)$ \\
\hline $\mathrm{Si}(1)-\mathrm{Cl}(3)$ & $2.0547(7)$ & $C(35)-C(36)$ & $1.388(3)$ \\
\hline $\mathrm{Si}(1)-\mathrm{Cl}(2)$ & $2.0797(7)$ & $\mathrm{N}(37)-\mathrm{C}(38)$ & $1.482(2)$ \\
\hline $\mathrm{C}(4)-\mathrm{N}(12)$ & $1.494(2)$ & $\mathrm{C}(38)-\mathrm{C}(39)$ & $1.528(3)$ \\
\hline \multirow[t]{2}{*}{$\mathrm{C}(4)-\mathrm{C}(5)$} & $1.574(3)$ & $\mathrm{C}(38)-\mathrm{C}(40)$ & $1.530(3)$ \\
\hline & & $\mathrm{C}(38)-\mathrm{C}(41)$ & $1.533(3)$ \\
\hline
\end{tabular}




\begin{tabular}{|c|c|}
\hline $\mathrm{N}(25)-\mathrm{Si}(1)-\mathrm{C}(4)$ & $121.19(8)$ \\
\hline $\mathrm{N}(25)-\mathrm{Si}(1)-\mathrm{Cl}(3)$ & $107.80(6)$ \\
\hline $\mathrm{C}(4)-\mathrm{Si}(1)-\mathrm{Cl}(3)$ & $109.51(6)$ \\
\hline $\mathrm{N}(25)-\mathrm{Si}(1)-\mathrm{Cl}(2)$ & $107.97(6)$ \\
\hline $\mathrm{C}(4)-\mathrm{Si}(1)-\mathrm{Cl}(2)$ & $105.65(6)$ \\
\hline $\mathrm{Cl}(3)-\mathrm{Si}(1)-\mathrm{Cl}(2)$ & $103.28(3)$ \\
\hline$N(12)-C(4)-C(5)$ & $106.72(14)$ \\
\hline $\mathrm{N}(12)-\mathrm{C}(4)-\mathrm{Si}(1)$ & $111.94(12)$ \\
\hline $\mathrm{C}(5)-\mathrm{C}(4)-\mathrm{Si}(1)$ & $117.73(12)$ \\
\hline $\mathrm{N}(12)-\mathrm{C}(4)-\mathrm{H}(4)$ & $109.0(11)$ \\
\hline $\mathrm{C}(5)-\mathrm{C}(4)-\mathrm{H}(4)$ & $108.7(11)$ \\
\hline $\mathrm{Si}(1)-\mathrm{C}(4)-\mathrm{H}(4)$ & $102.4(11)$ \\
\hline $\mathrm{C}(8)-\mathrm{C}(5)-\mathrm{C}(7)$ & $110.47(16)$ \\
\hline $\mathrm{C}(8)-\mathrm{C}(5)-\mathrm{C}(6)$ & 111.11(16) \\
\hline$C(7)-C(5)-C(6)$ & $107.05(16)$ \\
\hline$C(8)-C(5)-C(4)$ & $101.86(14)$ \\
\hline$C(7)-C(5)-C(4)$ & $110.87(15)$ \\
\hline$C(6)-C(5)-C(4)$ & $115.47(15)$ \\
\hline $\mathrm{C}(9)-\mathrm{C}(8)-\mathrm{C}(5)$ & $108.00(15)$ \\
\hline $\mathrm{N}(12)-\mathrm{C}(9)-\mathrm{C}(8)$ & $103.01(14)$ \\
\hline $\mathrm{N}(12)-\mathrm{C}(9)-\mathrm{C}(11)$ & $112.86(15)$ \\
\hline $\mathrm{C}(8)-\mathrm{C}(9)-\mathrm{C}(11)$ & $110.78(16)$ \\
\hline $\mathrm{N}(12)-\mathrm{C}(9)-\mathrm{C}(10)$ & $110.66(15)$ \\
\hline$C(8)-C(9)-C(10)$ & $112.03(16)$ \\
\hline$C(11)-C(9)-C(10)$ & $107.56(16)$ \\
\hline $\mathrm{C}(13)-\mathrm{N}(12)-\mathrm{C}(4)$ & $117.69(14)$ \\
\hline $\mathrm{C}(13)-\mathrm{N}(12)-\mathrm{C}(9)$ & $122.11(14)$ \\
\hline $\mathrm{C}(4)-\mathrm{N}(12)-\mathrm{C}(9)$ & $110.72(14)$ \\
\hline$C(21)-C(13)-C(14)$ & $118.76(16)$ \\
\hline$C(21)-C(13)-N(12)$ & $118.45(16)$ \\
\hline $\mathrm{C}(14)-\mathrm{C}(13)-\mathrm{N}(12)$ & $122.72(16)$ \\
\hline $\mathrm{C}(18)-\mathrm{C}(14)-\mathrm{C}(13)$ & $119.18(17)$ \\
\hline$C(18)-C(14)-C(15)$ & $116.56(16)$ \\
\hline$C(13)-C(14)-C(15)$ & $124.22(16)$ \\
\hline $\mathrm{C}(14)-\mathrm{C}(15)-\mathrm{C}(17)$ & $110.69(15)$ \\
\hline $\mathrm{C}(14)-\mathrm{C}(15)-\mathrm{C}(16)$ & $112.29(15)$ \\
\hline$C(17)-C(15)-C(16)$ & $108.46(16)$ \\
\hline
\end{tabular}

C(19)-C(18)-C(14)

C(18)-C(19)-C(20)

121.60(17)

119.21(18)

121.52(17)

$119.47(17)$

117.95(16)

122.57(17)

113.18(16)

109.85(15)

109.36(16)

115.81(14)

120.05(12)

123.46(12)

109.18(14)

108.85(15)

108.45(16)

112.11(16)

107.57(16)

110.60(16)

116.20(16)

129.12(17)

114.69(15)

118.72(17)

120.16(16)

121.08(17)

120.49(17)

120.19(19)

119.87(18)

120.06(18)

120.63(18)

128.55(16)

103.99(15)

118.33(16)

108.10(18)

106.35(16)

109.48(18)

110.24(17) 


\section{(S4) Theoretical calculation}

\section{Computational Details}

All calculations were performed in Gaussian09 quantum package. ${ }^{\mathrm{S}}$ All intermediates are optimized with the global-hybrid meta-GGA DFT functional, M06- $2 \mathrm{X}^{\mathrm{S9}}$ employing SVP basis sets for all atoms. ${ }^{\mathrm{S} 10}$ Geometries were optimized without any symmetry constraints. Harmonic force constants were computed at the optimized geometries to characterize the stationary points as minima or saddle points. Zero-point vibrational corrections were determined from the harmonic vibrational frequencies to convert the total energies $E_{e}$ to ground state energies $E_{0}$. The rigid-rotor harmonic-oscillator approximation was applied for evaluating the thermal and entropic contributions that are needed to derive the enthalpies $\mathrm{H}_{298}$ and Gibbs free energies, $\mathrm{G}_{298}$ at $298 \mathrm{~K}$. All transition states were located using the linear synchronous transit (LST) ${ }^{\mathrm{S} 11}$ method in which the reaction coordinate was kept fixed at different distances while all other degrees of freedom were relaxed. After the linear transit search the transition states were optimized using the default Berny algorithm implemented in the Gaussian09 code. ${ }^{\mathrm{S} 8}$ All transition states were confirmed by IRC (Intrinsic Reaction Coordinate) calculations. For further validation of the energy change, we have also performed single point calculation of optimized geometry incorporating higher basis set TZVPP ${ }^{S 12}$ for all atoms. Solvation energies in toluene $(\varepsilon=$ 2.3741) were evaluated by a self-consistent reaction field (SCRF) approach using the SMD continuum solvation model. ${ }^{\mathrm{S} 13} \Delta \mathrm{E}_{\mathrm{L}}{ }^{\mathrm{S}}$ is the solvent electronic energy at M06-2X/TZVPP/SMD//M06-2X/SVP level. $\Delta \mathrm{G}_{\mathrm{sol}}$ is the solvent-phase free energy change where the total solvent electronic energy $\left(\Delta \mathrm{E}_{\mathrm{L}}^{\mathrm{S}}\right)$ is augmented with the gas-phase free energy correction at M06-2X/SVP level. The $\Delta \mathrm{G}_{\text {sol }}$ values are reported everywhere in the manuscript. The charge distribution was analyzed using the Weinhold's NPA (Natural Population Analysis) approach. The wavefunction file generated from the quantum code, were used to perform QTAIM ${ }^{\text {S14 }}$ analysis in the AIMALL program suite. We have applied Bader's AIM (Atoms-in-molecule) ${ }^{\mathrm{S} 15}$ concept to characterize the electron distribution in 3a. Any bonded pair of atoms has a bond path, i.e; a connecting line with maximum electron density. The bond critical point (BCP) is a point on this line where the gradient $\nabla \rho(\mathrm{r})$ of the density is equal to zero. The magnitude of the electron density, $\rho(\mathrm{r})$ and its Laplacian, $\nabla^{2} \rho(\mathrm{r})$ at the BCP provide information about the strength and type of bond. The Laplacian indicates whether the density is locally concentrated $\left(\nabla^{2} \rho<0\right)$ or depleted $\left(\nabla^{2} \rho>0\right)$. Optimized geometries and orbital diagrams are illustrated using Chemcraft and CYLview drawing. ${ }^{\text {S16 }}$

Table S5. Some computed bond lengths $(\AA)$ of all intermediates and transition states.

\begin{tabular}{|l|c|c|c|c|c|c|c|}
\hline No. & Si1-N2 & Si1-C1 & Si1-C11 & Si1-C12 & Si1-H1 & C1-H1 & C1-N1 \\
\hline $\mathbf{1}$ & 1.709 & - & 2.074 & 2.069 & 1.472 & - & - \\
\hline cAAC & - & - & - & - & - & - & 1.309 \\
\hline
\end{tabular}




\begin{tabular}{|c|c|c|c|c|c|c|c|}
\hline Ia & 1.719 & 5.100 & 2.077 & 2.084 & 1.471 & 3.672 & 1.306 \\
\hline [Ia-IIa] & 1.759 & 3.310 & 2.148 & 2.153 & 1.816 & 1.497 & 1.297 \\
\hline IIa & 1.793 & 4.152 & 2.229 & 2.222 & 4.802 & 1.096 & 1.282 \\
\hline 2 & 1.733 & 1.921 & 2.091 & 2.093 & 2.398 & 1.105 & 1.475 \\
\hline IIIa & 1.814 & $3.181 \mathrm{a}$ & 2.236 & 2.404 & $3.455 b$ & 1.101 & 1.286 \\
\hline [IIIa-IVa] $^{*}$ & 1.830 & $2.412^{\mathrm{a}}$ & 2.195 & 2.550 & $2.340^{\mathrm{b}}$ & 1.094 & 1.365 \\
\hline IVa & 1.802 & $1.926^{\mathrm{a}}$ & 2.161 & 3.804 & $1.919^{b}$ & 1.097 & 1.383 \\
\hline cAAC.HCl & - & $1.912^{\mathrm{a}}$ & - & - & - & 1.098 & 1.392 \\
\hline $\mathbf{3 a}$ & 1.793 & - & 2.148 & - & $1.902^{b}$ & - & - \\
\hline lia & 1.799 & 2.207 & 2.134 & 2.151 & 1.479 & 2.481 & 1.306 \\
\hline$[1 \mathrm{ia}-2]^{\ddagger}$ & 1.776 & 1.915 & 2.094 & 2.153 & 1.633 & 1.745 & 1.336 \\
\hline Ib & 1.718 & 3.577 & 2.074 & 2.082 & 1.460 & 2.612 & 1.362 \\
\hline$[$ Ib-IIb] & 1.758 & 3.223 & 2.143 & 2.173 & 1.184 & 1.444 & 1.349 \\
\hline IIb & 1.780 & 3.453 & 2.197 & 2.235 & 2.378 & 1.122 & 1.334 \\
\hline$[\text { IIb-2b] }]^{*}$ & 1.777 & 2.549 & 2.167 & 2.155 & 2.671 & 1.090 & 1.3389 \\
\hline $2 \mathrm{~b}$ & 1.735 & 1.974 & 2.094 & 2.078 & 2.493 & 1.111 & 1.486 \\
\hline lib & 1.79 & 2.282 & 2.129 & 2.125 & 1.476 & 1.495 & 1.360 \\
\hline$[1 \mathrm{ib}-2 \mathrm{~b}]^{*}$ & 1.740 & 1.874 & 2.082 & 2.141 & 1.884 & 1.564 & 1.410 \\
\hline
\end{tabular}

${ }^{\mathrm{a}} \mathrm{C} 1-\mathrm{Cl} 1$ bond distance. ${ }^{\mathrm{b}}$ distance between Si1 and $\mathrm{C} 1$ of incoming $\mathbf{c A A C}$.

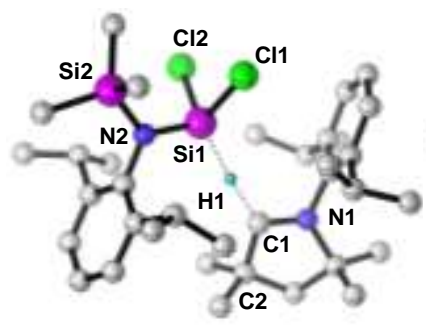

[Ia-IIa] $^{\ddagger}$

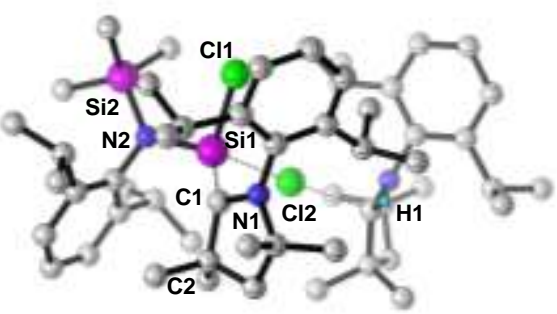

[IIIa-IVa]

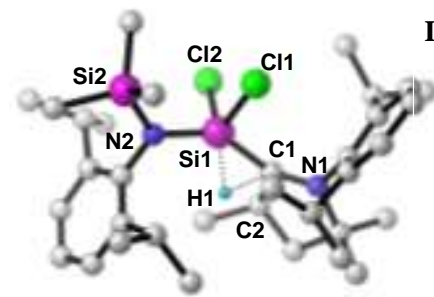

[1i-2 $]^{\ddagger}$

$\begin{array}{cccc}\text { Distance }(\AA) & {\text { IIa-IIa }]^{*}}^{[\text {IIIa-IVa }]^{\ddagger}} & {[1 \mathrm{i}-2]^{\ddagger}} \\ \text { Si1-N2 } & 1.759 & 1.830 & 1.776 \\ \text { Si1-Cl2 } & 2.153 & 2.550 & 2.152 \\ \text { Si1-H1 } & 1.816 & 2.340^{\mathrm{a}} & 1.633 \\ \text { Si1-C1 } & 3.310 & 2.412^{\mathrm{b}} & 1.915 \\ \text { C1-H1 } & 1.497 & 1.094 & 1.745\end{array}$

Figure S7. Optimized geometries of key transition states at M06-2X/SVP level. Hydrogen atoms except H1 are omitted for clarity. ${ }^{\mathrm{a}}$ Distance between $\mathrm{Si} 1$ and $\mathrm{C} 1$ of incoming $\mathbf{c A A C} .{ }^{\mathrm{b}} \mathrm{C} 1-\mathrm{Cl} 2$ bond distance. 
Table S6. NPA charges of selected atoms for all intermediates and transition states.

\begin{tabular}{|c|c|c|c|c|c|c|c|c|}
\hline No. & $\mathrm{N} 2$ & Si1 & Cl1 & $\mathrm{Cl} 2$ & $\mathrm{H} 1$ & $\mathrm{C} 1$ & $\mathrm{C} 2$ & N1 \\
\hline 1 & -1.349 & 1.522 & -0.393 & -0.387 & -0.185 & - & - & - \\
\hline cAAC & - & - & - & - & - & 0.152 & -0.190 & -0.446 \\
\hline Ia & -1.364 & 1.571 & -0.401 & -0.408 & -0.182 & 0.165 & -0.197 & -0.453 \\
\hline [Ia-IIa] $^{*}$ & -1.373 & 1.246 & -0.478 & -0.481 & 0.049 & 0.269 & -0.185 & -0.410 \\
\hline IIa & -1.398 & 0.877 & -0.559 & -0.557 & 0.235 & 0.329 & -0.132 & -0.274 \\
\hline 2 & -1.406 & 1.918 & -0.421 & -0.436 & 0.226 & -0.537 & -0.050 & -0.525 \\
\hline IIIa & -1.430 & 1.036 & -0.585 & -0.671 & 0.295 & $\begin{array}{c}0.345 \\
0.221^{\mathrm{a}}\end{array}$ & $\begin{array}{l}-0.143 \\
-0.203^{\mathrm{a}}\end{array}$ & $\begin{array}{c}-0.320 \\
-0.467^{\mathrm{a}}\end{array}$ \\
\hline [IIIa-IVa] & -1.422 & 1.313 & -0.534 & -0.481 & 0.212 & $\begin{array}{c}0.203 \\
0.107^{\mathrm{a}}\end{array}$ & $\begin{array}{l}-0.131 \\
-0.181^{\mathrm{a}}\end{array}$ & $\begin{array}{l}-0.472 \\
-0.457^{\mathrm{a}}\end{array}$ \\
\hline IVa & -1.385 & 1.240 & -0.474 & -0.224 & 0.206 & $\begin{array}{c}0.108 \\
-0.124^{\mathrm{a}}\end{array}$ & $\begin{array}{l}-0.092 \\
-0.128^{\mathrm{a}}\end{array}$ & $\begin{array}{l}-0.503 \\
-0.432^{\mathrm{a}}\end{array}$ \\
\hline cAAC.HCl & - & - & - & -0.225 & 0.205 & 0.099 & -0.082 & -0.495 \\
\hline $\mathbf{3 a}$ & -1.366 & 1.230 & -0.457 & - & - & $-0.133^{\mathrm{a}}$ & $-0.140^{\mathrm{a}}$ & $-0.445^{\mathrm{a}}$ \\
\hline 1ia & -1.364 & 1.520 & -0.429 & -0.423 & -0.245 & 0.292 & -0.211 & -0.478 \\
\hline$[1 \text { ia-2 }]^{\ddagger}$ & -1.370 & 1.690 & -0.451 & -0.403 & -0.127 & -0.072 & -0.130 & -0.473 \\
\hline Ib & -1.369 & 1.598 & -0.411 & -0.421 & -0.191 & 0.143 & $-0.439^{b}$ & -0.440 \\
\hline [Ib-IIIb] $^{*}$ & -1.388 & 1.261 & -0.482 & -0.508 & 0.059 & 0.206 & $-0.382^{b}$ & -0.383 \\
\hline IIb & -1.397 & 0.947 & -0.539 & -0.563 & 0.278 & 0.246 & $-0.332^{b}$ & -0.325 \\
\hline$[\mathbf{I I b}-\mathbf{2 b}]^{*}$ & -1.405 & 1.283 & -0.499 & -0.486 & 0.229 & 0.175 & $-0.428^{\mathrm{b}}$ & -0.434 \\
\hline $2 b$ & -1.383 & 1.848 & -0.424 & -0.415 & 0.206 & -0.284 & $-0.486^{\mathrm{b}}$ & -0.497 \\
\hline 1ib & -1.366 & 1.502 & -0.430 & -0.427 & -0.235 & 0.213 & $-0.413^{b}$ & -0.410 \\
\hline$[1 \mathrm{ib}-2 \mathrm{~b}]^{\ddagger}$ & -1.380 & 1.782 & -0.389 & -0.444 & -0.115 & -0.121 & $-0.456^{b}$ & -0.446 \\
\hline
\end{tabular}

${ }^{a}$ NPA charge of incoming cAAC. ${ }^{b}$ NPA charge of N' of NHC. 


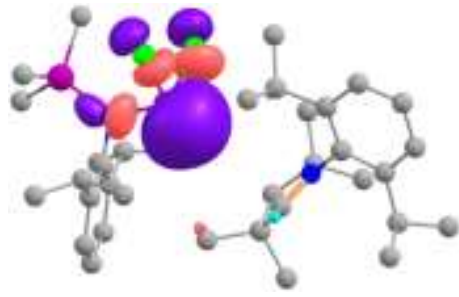

IIa-HOMO

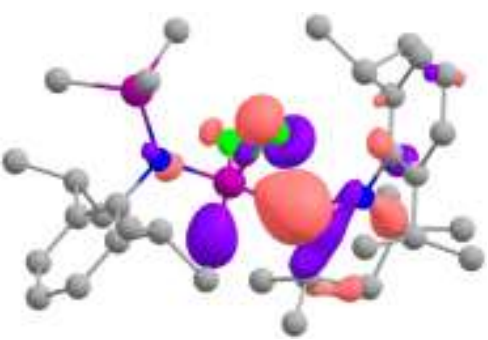

1ia-HOMO-3

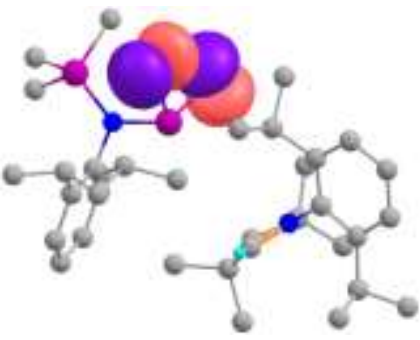

IIa-HOMO-4

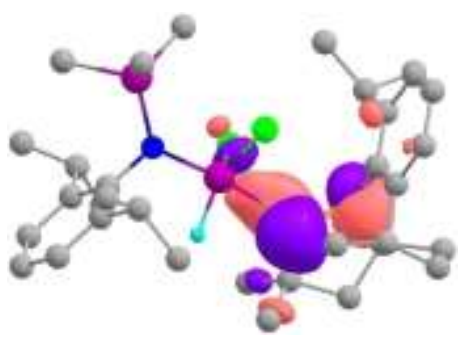

1ia-LUMO

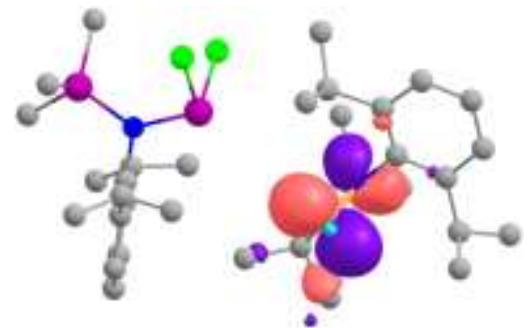

IIa-LUMO

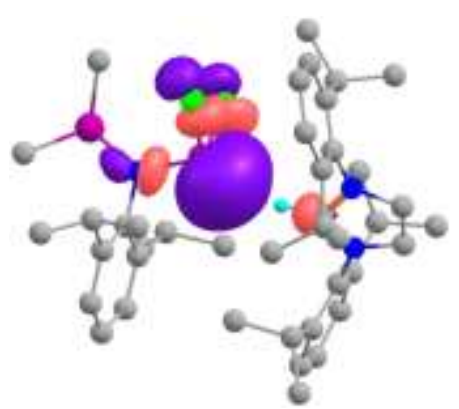

IIb-HOMO

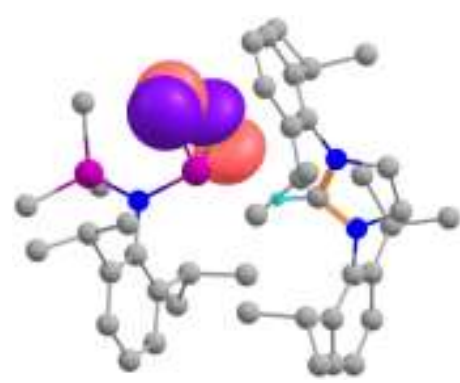

IIb-HOMO-5

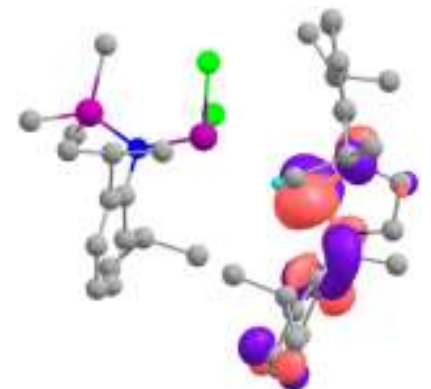

IIb-LUMO

Figure S8. KS-MO of some intermediates (isosurface $=0.05 \mathrm{au}$ ). Hydrogen atoms are omitted for clarity. 


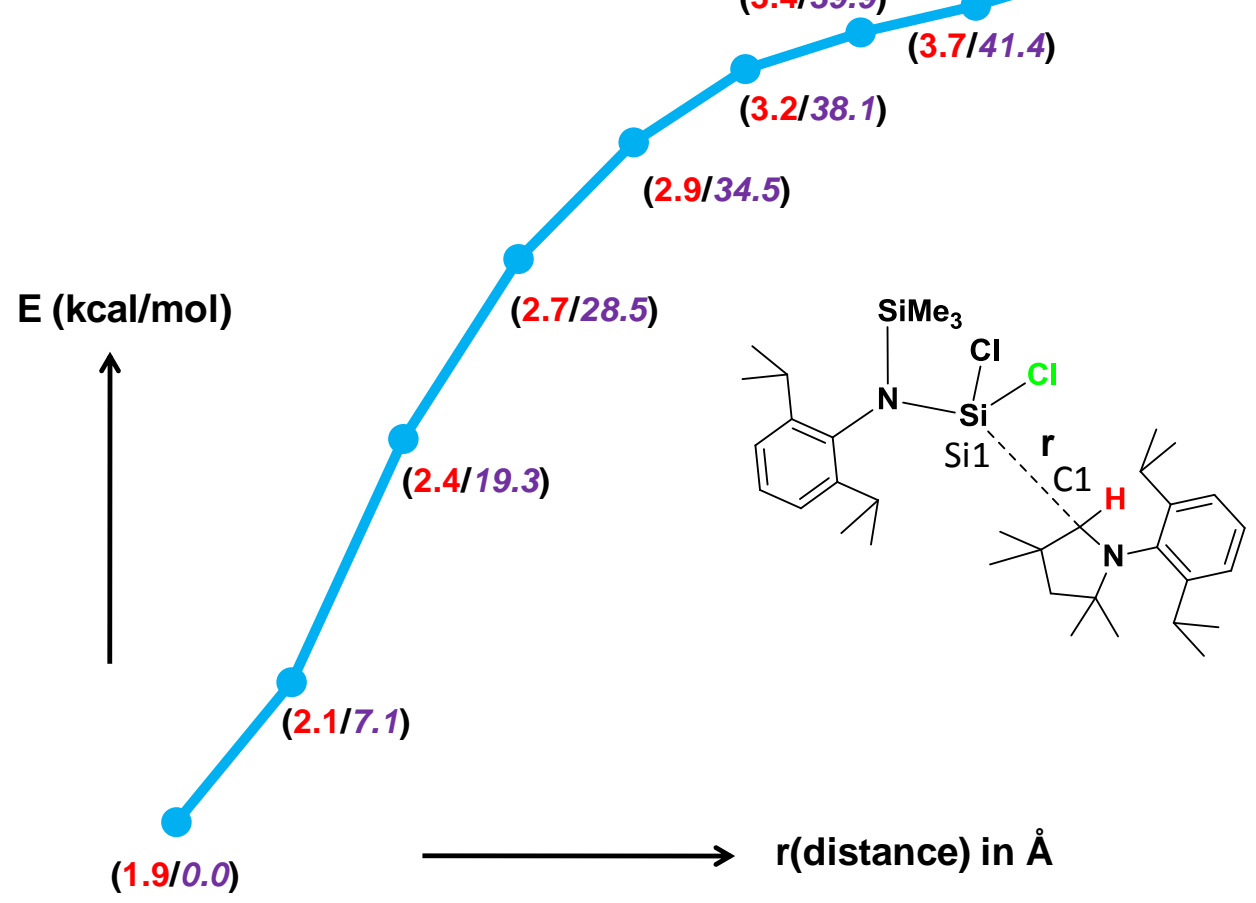

Figure S9. Potential energy surface profile of Si1 progress towards C1. The values in parentheses (Si1$\mathrm{C} 1 /$ relative energies) are calculated at M06-2X/SVP level. The energy levels are not drawn to the scale.

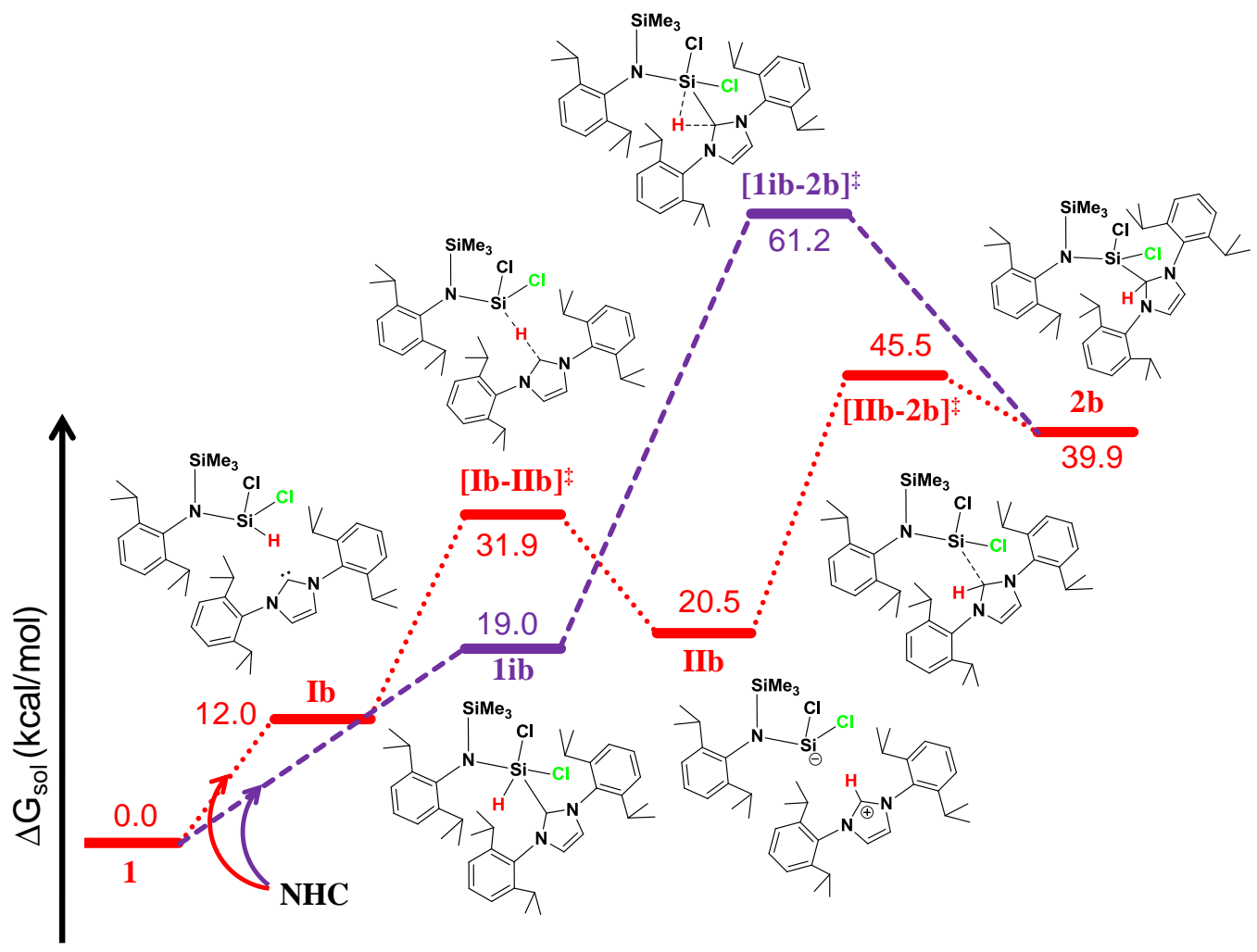

Figure S10. Energy profile diagram formation of Si-H activated product for NHC. 


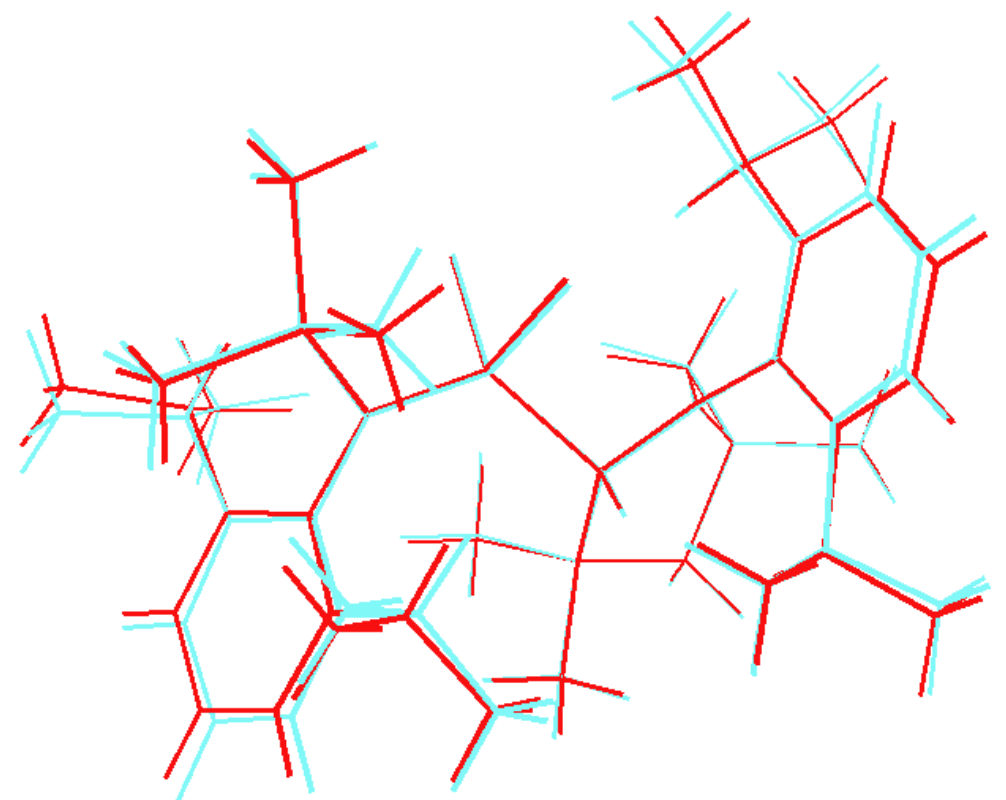

Figure S11. Superposition of crystal structure of 2 with the optimized geometry at R-M06-2X/SVP level of theory.

\section{QTAIM Study:}

The optimized geometry of $\mathbf{2}$ is in good agreement with the crystal structure (Figure 1, S11). According to NBO results, it is observed that in $2 \mathrm{Si} 1$ is connected to $\mathrm{N} 2, \mathrm{Cl} 1, \mathrm{Cl} 2$, and $\mathrm{C} 1$ via single bonds with electron occupancy of 1.852, 1.974, 1.974 and $1.951 \mathrm{e}$, respectively. The electron density of the Si1-C1 bond is mostly localized on the $\mathrm{C} 1$ center (73\%), which indicates either closed-shell interaction or polar electron-sharing bond. For further support, a topological analysis was performed on the optimized geometry of $\mathbf{2}$ according to Bader's QTAIM method ${ }^{\text {S17 }}$ to support the bonding natures obtain from NBO analysis. The electron density on the Sil-C1 bond $(\rho(\mathrm{r})=0.122$ a.u. Table S6) is significantly higher than that in the $\mathrm{C} \rightarrow \mathrm{Si}$ donor-acceptor-type bond in (cAAC) ${ }_{2} \mathrm{Si}(0.108$ a.u. $)^{S 18}$. However, the density is comparable to the one in octamethyl-cyclotetrasilazane $(0.128$ a.u. $)$ as studied by Kocher et al. ${ }^{\mathrm{S} 19}$ The Laplacian of $\nabla^{2} \rho(\mathrm{r})=+0.065$ a.u. indicates only small depletion of the density. Furthermore, the calculated ellipticity $\left(\varepsilon_{\mathrm{BCP}}=0.068\right)$ is much lower than that of the $\mathrm{C} \rightarrow \mathrm{Si}$ donor-acceptor-type bond $\left(\varepsilon_{\mathrm{BCP}}=0.56\right)$ indicating its covalent nature. ${ }^{\mathrm{S} 17}$ The same result is demonstrated by the delocalization index (DI) value of $\delta(\mathrm{Si} 1, \mathrm{C} 1), 0.51$ similar with the DI value of $\mathrm{Si}-\mathrm{C}$ bond in $\mathrm{H}_{3} \mathrm{Si}-\mathrm{CH}_{3}(0.53)$.

Table S7. BCP (bond critical point) parameters for some important bonds in $\mathbf{2}$.

\begin{tabular}{|c|c|c|c|c|}
\hline Atoms & $\rho($ r), a.u. & $\nabla^{2} \rho($ r), a.u. & $\varepsilon$ & DI \\
\hline Si1-N2 & 0.131 & +0.523 & 0.161 & 0.46 \\
\hline Si1-C1 & 0.122 & +0.523 & 0.068 & 0.51 \\
\hline
\end{tabular}




\begin{tabular}{|c|c|c|c|c|}
\hline Si1-C11 & 0.093 & +0.198 & 0.035 & 0.39 \\
\hline Si1-Cl2 & 0.093 & +0.190 & 0.041 & 0.41 \\
\hline C1-H1 & 0.278 & -0.990 & 0.042 & 0.89 \\
\hline C1-N1 & 0.253 & -0.559 & 0.099 & 1.00 \\
\hline
\end{tabular}

Table S6. Cartesian coordinates (in $\AA$ ) of optimized geometries of all the intermediates and transition states at R/U-M06-2X/def2-SVP level of theory and the energies E (in a.u.) at the M06-2X/TZVPP//M06-2X/SVP level of theory using G09 program package.

\begin{tabular}{|lrrr|}
\hline $\mathbf{1}$ & \multicolumn{4}{|c|}{} \\
$\mathbf{4 7}$ & \multicolumn{4}{l}{} \\
$\mathrm{E}_{\mathrm{e}}=-2142.14977203$ & & \\
$\mathrm{Si}$ & 1.40404 & 0.18903 & -1.19741 \\
$\mathrm{Si}$ & 0.91431 & 0.09598 & 1.86288 \\
$\mathrm{C}$ & -3.25669 & 0.76639 & -0.41347 \\
$\mathrm{H}$ & -3.93225 & 1.62085 & -0.48234 \\
$\mathrm{C}$ & -3.76506 & -0.52098 & -0.55678 \\
$\mathrm{H}$ & -4.83174 & -0.67241 & -0.72881 \\
$\mathrm{C}$ & -2.90667 & -1.61180 & -0.49168 \\
$\mathrm{H}$ & -3.30660 & -2.61919 & -0.62224 \\
$\mathrm{C}$ & -1.03468 & -0.13332 & -0.10632 \\
$\mathrm{C}$ & -1.89438 & 0.98494 & -0.19289 \\
$\mathrm{C}$ & -1.35721 & 2.40555 & -0.13134 \\
$\mathrm{H}$ & -0.36200 & 2.36744 & 0.33383 \\
$\mathrm{C}$ & -1.53543 & -1.44333 & -0.26839 \\
$\mathrm{C}$ & -0.62338 & -2.66010 & -0.27401 \\
$\mathrm{H}$ & 0.34244 & -2.35744 & 0.14967 \\
$\mathrm{C}$ & -1.17546 & 2.95589 & -1.55198 \\
$\mathrm{H}$ & -2.14774 & 3.01745 & -2.06504 \\
$\mathrm{H}$ & -0.51912 & 2.31401 & -2.15742 \\
$\mathrm{H}$ & -0.73047 & 3.96165 & -1.52621 \\
$\mathrm{C}$ & -2.23416 & 3.34472 & 0.69917 \\
$\mathrm{H}$ & -2.44066 & 2.92840 & 1.69552 \\
$\mathrm{H}$ & -3.19863 & 3.53975 & 0.20681 \\
$\mathrm{H}$ & -1.73316 & 4.31555 & 0.82579 \\
$\mathrm{C}$ & -1.16942 & -3.81094 & 0.57517 \\
$\mathrm{H}$ & -1.45130 & -3.47381 & 1.58316 \\
$\mathrm{H}$ & -0.41134 & -4.60153 & 0.67459 \\
$\mathrm{H}$ & -2.05877 & -4.26666 & 0.11428 \\
$\mathrm{~N}$ & 0.36757 & 0.07575 & 0.15681 \\
$\mathrm{C}$ & 1.16509 & -1.64995 & 2.50700 \\
$\mathrm{H}$ & 1.91058 & -2.18766 & 1.90180 \\
$\mathrm{H}$ & 0.23000 & -2.22814 & 2.50342 \\
$\mathrm{H}$ & 1.53242 & -1.60723 & 3.54420 \\
$\mathrm{C}$ & -0.39664 & 0.95615 & 2.89128 \\
$\mathrm{H}$ & -0.15041 & 0.85087 & 3.95902 \\
$\mathrm{H}$ & -1.39079 & 0.51374 & 2.72594 \\
$\mathrm{H}$ & -0.45290 & 2.02968 & 2.66029 \\
$\mathrm{C}$ & 2.54453 & 1.01832 & 1.95103 \\
$\mathrm{H}$ & 2.43967 & 2.06711 & 1.63984 \\
$\mathrm{H}$ & 3.31785 & 0.55109 & 1.32288 \\
$\mathrm{H}$ & 2.90226 & 0.99845 & 2.99213 \\
\hline & & & \\
\hline
\end{tabular}

\begin{tabular}{|c|c|c|c|}
\hline $\mathrm{C}$ & -0.36155 & -3.13316 & -1.70947 \\
\hline $\mathrm{H}$ & 0.31856 & -3.99785 & -1.71227 \\
\hline $\mathrm{H}$ & 0.09543 & -2.34281 & -2.32206 \\
\hline $\mathrm{H}$ & -1.30288 & -3.43112 & -2.19676 \\
\hline $\mathrm{Cl}$ & 2.91036 & -1.23047 & -1.05957 \\
\hline $\mathrm{Cl}$ & 2.36283 & 2.01841 & -1.32544 \\
\hline $\mathrm{H}$ & 0.66410 & -0.01573 & -2.45309 \\
\hline \multicolumn{4}{|c|}{ cAAC } \\
\hline \multicolumn{4}{|c|}{$\begin{array}{l}52 \\
E_{e}=-835.429640351\end{array}$} \\
\hline $\mathrm{C}$ & -2.69086 & -0.63715 & 0.94541 \\
\hline $\mathrm{C}$ & -2.77275 & -0.31190 & -0.56789 \\
\hline $\mathrm{C}$ & -1.33472 & -0.14912 & -1.04002 \\
\hline $\mathrm{C}$ & -1.28910 & -0.19753 & 1.39484 \\
\hline $\mathrm{H}$ & -3.47882 & -0.14209 & 1.53225 \\
\hline $\mathrm{H}$ & -2.80051 & -1.72216 & 1.09685 \\
\hline $\mathrm{N}$ & -0.60204 & -0.09782 & 0.04345 \\
\hline $\mathrm{C}$ & 0.82689 & 0.07578 & -0.01662 \\
\hline $\mathrm{C}$ & 1.64579 & -1.06808 & -0.06319 \\
\hline $\mathrm{C}$ & 1.36780 & 1.37401 & -0.05674 \\
\hline $\mathrm{C}$ & 3.03162 & -0.88635 & -0.03678 \\
\hline $\mathrm{C}$ & 2.76025 & 1.50588 & -0.02868 \\
\hline $\mathrm{C}$ & 3.58703 & 0.38842 & 0.00656 \\
\hline $\mathrm{H}$ & 3.68757 & -1.75795 & -0.06899 \\
\hline $\mathrm{H}$ & 3.20434 & 2.50231 & -0.05518 \\
\hline $\mathrm{H}$ & 4.67081 & 0.51140 & 0.03480 \\
\hline $\mathrm{C}$ & 1.06826 & -2.45611 & -0.28689 \\
\hline $\mathrm{H}$ & 0.00171 & -2.42672 & -0.03215 \\
\hline $\mathrm{C}$ & 0.49729 & 2.59830 & -0.28583 \\
\hline $\mathrm{H}$ & -0.53258 & 2.33828 & -0.01758 \\
\hline $\mathrm{C}$ & 0.48967 & 2.92722 & -1.78448 \\
\hline $\mathrm{H}$ & -0.15923 & 3.79309 & -1.98612 \\
\hline $\mathrm{H}$ & 1.50580 & 3.16894 & -2.13288 \\
\hline $\mathrm{H}$ & 0.11768 & 2.06608 & -2.35769 \\
\hline $\mathrm{C}$ & 0.91194 & 3.80770 & 0.55212 \\
\hline $\mathrm{H}$ & 1.89512 & 4.19566 & 0.24658 \\
\hline $\mathrm{H}$ & 0.18719 & 4.62430 & 0.42061 \\
\hline $\mathrm{H}$ & 0.96346 & 3.56284 & 1.62343 \\
\hline $\mathrm{C}$ & 1.73067 & -3.53398 & 0.57104 \\
\hline $\mathrm{H}$ & 1.20887 & -4.49366 & 0.44313 \\
\hline $\mathrm{H}$ & 2.77991 & -3.69479 & 0.28185 \\
\hline $\mathrm{H}$ & 1.71067 & -3.26912 & 1.63857 \\
\hline
\end{tabular}




\begin{tabular}{|c|c|c|c|c|c|c|c|}
\hline & & & & & & & \\
\hline $\mathrm{C}$ & 1.14894 & -2.79228 & -1.78160 & $\mathrm{H}$ & -1.65110 & -3.97245 & -0.49273 \\
\hline $\mathrm{H}$ & 2.19667 & -2.80605 & -2.11962 & $\mathrm{C}$ & -2.08638 & -0.39616 & -1.97610 \\
\hline $\mathrm{H}$ & 0.70954 & -3.78174 & -1.97957 & $\mathrm{C}$ & -1.41677 & -1.39473 & -2.68706 \\
\hline $\mathrm{H}$ & 0.60280 & -2.03983 & -2.36839 & $\mathrm{H}$ & -0.93725 & -1.15064 & -3.63606 \\
\hline $\mathrm{C}$ & -3.45358 & -1.43068 & -1.35750 & $\mathrm{C}$ & -2.00703 & 1.04811 & -2.44394 \\
\hline $\mathrm{H}$ & -4.48810 & -1.57723 & -1.00846 & $\mathrm{H}$ & -2.78600 & 1.62217 & -1.92706 \\
\hline $\mathrm{H}$ & -3.47223 & -1.18531 & -2.42837 & $\mathrm{C}$ & -1.65408 & -2.20305 & 2.14759 \\
\hline $\mathrm{H}$ & -2.91140 & -2.38122 & -1.23827 & $\mathrm{H}$ & -1.21580 & -1.19837 & 2.05398 \\
\hline $\mathrm{C}$ & -3.48966 & 1.01790 & -0.84523 & $\mathrm{H}$ & -0.89358 & -2.94234 & 1.85250 \\
\hline $\mathrm{H}$ & -3.44502 & 1.25685 & -1.91691 & $\mathrm{H}$ & -1.91247 & -2.37262 & 3.20431 \\
\hline $\mathrm{H}$ & -4.54582 & 0.95015 & -0.54157 & $\mathrm{C}$ & -3.58198 & -3.67715 & 1.43031 \\
\hline $\mathrm{H}$ & -3.02823 & 1.85299 & -0.29721 & $\mathrm{H}$ & -2.89273 & -4.50585 & 1.21055 \\
\hline $\mathrm{C}$ & -0.60962 & -1.21627 & 2.30905 & $\mathrm{H}$ & -4.45517 & -3.77986 & 0.76921 \\
\hline $\mathrm{H}$ & -0.62639 & -2.22787 & 1.88406 & $\mathrm{H}$ & -3.92040 & -3.80273 & 2.46916 \\
\hline $\mathrm{H}$ & 0.43409 & -0.93618 & 2.51589 & $\mathrm{C}$ & -2.89993 & -2.31937 & 1.26020 \\
\hline $\mathrm{H}$ & -1.14991 & -1.24696 & 3.26628 & $\mathrm{H}$ & -3.59984 & -1.54435 & 1.59269 \\
\hline $\mathrm{C}$ & -1.28609 & 1.16056 & 2.10194 & $\mathrm{C}$ & -0.65971 & 1.63330 & -1.99838 \\
\hline $\mathrm{H}$ & -0.25839 & 1.52959 & 2.23825 & $\mathrm{H}$ & -0.54371 & 2.66945 & -2.35237 \\
\hline $\mathrm{H}$ & -1.86189 & 1.91423 & 1.54925 & $\mathrm{H}$ & 0.16947 & 1.03627 & -2.41090 \\
\hline \multirow[t]{2}{*}{$\mathrm{H}$} & -1.74208 & 1.04906 & 3.09606 & $\mathrm{H}$ & -0.57293 & 1.63075 & -0.89987 \\
\hline & & & & $\mathrm{C}$ & -2.21983 & 1.20875 & -3.94900 \\
\hline \multicolumn{4}{|l|}{ Ia } & $\mathrm{H}$ & -2.25913 & 2.27546 & -4.21334 \\
\hline 99 & & & & $\mathrm{H}$ & -3.15720 & 0.73548 & -4.27750 \\
\hline \multicolumn{4}{|c|}{$E_{e}=-2977.59023031$} & $\mathrm{H}$ & -1.39479 & 0.76302 & -4.52422 \\
\hline $\mathrm{C}$ & -2.86366 & 1.03311 & 0.85016 & $\mathrm{C}$ & 2.70101 & 1.95990 & -0.68846 \\
\hline $\mathrm{H}$ & 0.14943 & -0.72014 & -0.30340 & $\mathrm{C}$ & -1.29823 & -2.68426 & -2.17535 \\
\hline $\mathrm{Si}$ & 1.51217 & -1.26270 & -0.41304 & $\mathrm{H}$ & -0.75232 & -3.44438 & -2.73629 \\
\hline $\mathrm{C}$ & -3.95895 & 1.94396 & 1.38282 & $\mathrm{C}$ & 3.65190 & 1.51828 & -1.79219 \\
\hline $\mathrm{Si}$ & 4.22970 & -0.89245 & 1.03117 & $\mathrm{H}$ & 3.86488 & 0.45070 & -1.64487 \\
\hline $\mathrm{C}$ & -4.91641 & 0.41725 & -0.29671 & $\mathrm{C}$ & 1.50222 & 1.61982 & 1.42770 \\
\hline $\mathrm{C}$ & -5.17242 & 1.74197 & 0.43933 & $\mathrm{C}$ & 1.19675 & 0.78577 & 2.66118 \\
\hline $\mathrm{H}$ & -6.13308 & 1.72900 & 0.97519 & $\mathrm{H}$ & 1.22440 & -0.27590 & 2.36845 \\
\hline $\mathrm{H}$ & -5.21381 & 2.56441 & -0.29146 & $\mathrm{C}$ & 3.04972 & 1.68416 & -3.19110 \\
\hline $\mathrm{C}$ & -3.46195 & 3.39064 & 1.38215 & $\mathrm{H}$ & 2.84219 & 2.74323 & -3.40910 \\
\hline $\mathrm{H}$ & -2.58540 & 3.49179 & 2.03810 & $\mathrm{H}$ & 2.11570 & 1.12004 & -3.30116 \\
\hline $\mathrm{H}$ & -4.25079 & 4.07286 & 1.73664 & $\mathrm{H}$ & 3.75654 & 1.31971 & -3.95155 \\
\hline $\mathrm{H}$ & -3.16236 & 3.70276 & 0.36995 & $\mathrm{C}$ & 4.97318 & 2.29407 & -1.70913 \\
\hline $\mathrm{C}$ & -4.25331 & 1.50577 & 2.82545 & $\mathrm{H}$ & 5.42253 & 2.23020 & -0.71013 \\
\hline $\mathrm{H}$ & -4.60260 & 0.46364 & 2.87326 & $\mathrm{H}$ & 4.81168 & 3.35960 & -1.93458 \\
\hline $\mathrm{H}$ & -5.03115 & 2.14874 & 3.26558 & $\mathrm{H}$ & 5.69466 & 1.90298 & -2.44231 \\
\hline $\mathrm{H}$ & -3.34493 & 1.58227 & 3.44004 & $\mathrm{C}$ & 2.29897 & 1.01764 & 3.70469 \\
\hline $\mathrm{C}$ & 2.23838 & 3.28141 & -0.70263 & $\mathrm{H}$ & 3.30180 & 0.82473 & 3.29893 \\
\hline $\mathrm{H}$ & 2.53521 & 3.93840 & -1.52328 & $\mathrm{H}$ & 2.14925 & 0.37169 & 4.58352 \\
\hline $\mathrm{C}$ & -5.23806 & 0.48882 & -1.78862 & $\mathrm{H}$ & 2.27656 & 2.06584 & 4.04127 \\
\hline $\mathrm{H}$ & -4.75697 & 1.34685 & -2.27488 & $\mathrm{~N}$ & 2.67797 & -0.28033 & 0.38173 \\
\hline $\mathrm{H}$ & -6.32514 & 0.60140 & -1.90977 & $\mathrm{C}$ & 3.98047 & -1.95421 & 2.56349 \\
\hline $\mathrm{H}$ & -4.93079 & -0.43065 & -2.30872 & $\mathrm{H}$ & 3.46425 & -2.89261 & 2.32280 \\
\hline $\mathrm{C}$ & 1.41036 & 3.76894 & 0.29944 & $\mathrm{H}$ & 3.40405 & -1.43336 & 3.34049 \\
\hline $\mathrm{H}$ & 1.04787 & 4.79743 & 0.26124 & $\mathrm{H}$ & 4.96781 & -2.20340 & 2.98421 \\
\hline $\mathrm{C}$ & -5.68349 & -0.76228 & 0.30677 & $\mathrm{C}$ & 5.31851 & 0.58475 & 1.46023 \\
\hline $\mathrm{H}$ & -5.33306 & -1.71455 & -0.11902 & $\mathrm{H}$ & 5.78715 & 0.42994 & 2.44356 \\
\hline $\mathrm{H}$ & -6.75202 & -0.65657 & 0.07101 & $\mathrm{H}$ & 4.74967 & 1.52575 & 1.49977 \\
\hline $\mathrm{H}$ & -5.58019 & -0.80521 & 1.39885 & $\mathrm{H}$ & 6.12358 & 0.70320 & 0.72086 \\
\hline $\mathrm{C}$ & 1.05576 & 2.94175 & 1.36057 & $\mathrm{C}$ & 5.03179 & -1.95431 & -0.29589 \\
\hline $\mathrm{H}$ & 0.41821 & 3.33097 & 2.15511 & $\mathrm{H}$ & 5.24376 & -1.37266 & -1.20549 \\
\hline $\mathrm{C}$ & -2.67793 & -0.74439 & -0.74652 & $\mathrm{H}$ & 4.38419 & -2.79954 & -0.57395 \\
\hline $\mathrm{C}$ & -2.50720 & -2.01815 & -0.17625 & $\mathrm{H}$ & 5.98191 & -2.36702 & 0.07725 \\
\hline $\mathrm{C}$ & 2.30905 & 1.11763 & 0.37743 & $\mathrm{C}$ & -0.17518 & 1.07234 & 3.27299 \\
\hline $\mathrm{C}$ & -1.81376 & -2.98112 & -0.91910 & $\mathrm{H}$ & -0.40132 & 0.32281 & 4.04669 \\
\hline
\end{tabular}




\begin{tabular}{|c|c|c|c|c|c|c|c|}
\hline & & & & & & \multirow{2}{*}{\multicolumn{2}{|c|}{-0.55381}} \\
\hline $\mathrm{H}$ & -0.96677 & 1.04674 & 2.50818 & $\mathrm{H}$ & -2.49948 & & \\
\hline $\mathrm{H}$ & -0.19443 & 2.05740 & 3.76613 & $\mathrm{C}$ & -1.82394 & 0.64021 & -3.24688 \\
\hline $\mathrm{N}$ & -3.42855 & 0.26053 & -0.03931 & $\mathrm{H}$ & -1.83547 & 1.50178 & -3.93227 \\
\hline $\mathrm{Cl}$ & 1.56190 & -3.19025 & 0.35868 & $\mathrm{H}$ & -1.61369 & -0.26865 & -3.82935 \\
\hline \multirow[t]{2}{*}{$\mathrm{Cl}$} & 1.99455 & -1.52124 & -2.42411 & $\mathrm{H}$ & -0.98582 & 0.76182 & -2.54649 \\
\hline & & & & $\mathrm{C}$ & -4.33209 & 0.48639 & -3.48582 \\
\hline \multicolumn{4}{|c|}{$[\text { Ia-IIa }]^{\ddagger}$} & $\mathrm{H}$ & -4.37734 & 1.42476 & -4.05749 \\
\hline \multirow{2}{*}{\multicolumn{4}{|c|}{$\begin{array}{l}99 \\
E_{e}=-2977.56059642\end{array}$}} & $\mathrm{H}$ & -5.29181 & 0.34796 & -2.96609 \\
\hline & & & & $\mathrm{H}$ & -4.22161 & -0.33141 & -4.21245 \\
\hline $\mathrm{C}$ & -1.52483 & 1.32297 & 0.11595 & $\mathrm{C}$ & 3.07045 & 1.74255 & -0.41003 \\
\hline $\mathrm{H}$ & -0.44694 & 0.38038 & -0.32248 & $\mathrm{C}$ & -3.33950 & -3.07199 & -1.26546 \\
\hline $\mathrm{Si}$ & 0.77183 & -0.88535 & -0.78007 & $\mathrm{H}$ & -3.46894 & -4.06492 & -1.69759 \\
\hline C & -1.59157 & 2.80897 & 0.37817 & $\mathrm{C}$ & 3.25009 & 1.53471 & -1.90487 \\
\hline $\mathrm{Si}$ & 3.71791 & -1.78161 & -0.05795 & $\mathrm{H}$ & 3.27448 & 0.45101 & -2.08765 \\
\hline $\mathrm{C}$ & -3.75576 & 1.72609 & 0.86162 & $\mathrm{C}$ & 2.40701 & 0.94021 & 1.80539 \\
\hline $\mathrm{C}$ & -3.11305 & 3.07871 & 0.50960 & $\mathrm{C}$ & 1.83919 & -0.12020 & 2.73420 \\
\hline $\mathrm{H}$ & -3.34296 & 3.84545 & 1.26279 & $\mathrm{H}$ & 1.78620 & -1.06333 & 2.17285 \\
\hline $\mathrm{H}$ & -3.50841 & 3.43058 & -0.45660 & $\mathrm{C}$ & 2.04204 & 2.09478 & -2.66484 \\
\hline $\mathrm{C}$ & -0.98180 & 3.53214 & -0.82962 & $\mathrm{H}$ & 1.99353 & 3.19063 & -2.55239 \\
\hline $\mathrm{H}$ & 0.09689 & 3.32826 & -0.89092 & $\mathrm{H}$ & 1.10047 & 1.66979 & -2.28672 \\
\hline $\mathrm{H}$ & -1.12800 & 4.61804 & -0.72347 & $\mathrm{H}$ & 2.10730 & 1.85374 & -3.73621 \\
\hline $\mathrm{H}$ & -1.44944 & 3.20953 & -1.77267 & $\mathrm{C}$ & 4.54561 & 2.14349 & -2.44680 \\
\hline $\mathrm{C}$ & -0.78409 & 3.18306 & 1.62794 & $\mathrm{H}$ & 5.42268 & 1.81037 & -1.87365 \\
\hline $\mathrm{H}$ & -1.19841 & 2.73948 & 2.54284 & $\mathrm{H}$ & 4.52249 & 3.24351 & -2.41646 \\
\hline $\mathrm{H}$ & -0.78444 & 4.27722 & 1.74512 & $\mathrm{H}$ & 4.68813 & 1.85034 & -3.49717 \\
\hline $\mathrm{H}$ & 0.25828 & 2.84713 & 1.52532 & $\mathrm{C}$ & 2.72065 & -0.34319 & 3.96604 \\
\hline $\mathrm{C}$ & 3.32772 & 2.99839 & 0.14810 & $\mathrm{H}$ & 3.76597 & -0.53052 & 3.68038 \\
\hline $\mathrm{H}$ & 3.68196 & 3.80647 & -0.49526 & $\mathrm{H}$ & 2.36094 & -1.20654 & 4.54562 \\
\hline $\mathrm{C}$ & -5.10199 & 1.48186 & 0.19070 & $\mathrm{H}$ & 2.70941 & 0.53294 & 4.63270 \\
\hline $\mathrm{H}$ & -5.05557 & 1.61660 & -0.89743 & $\mathrm{~N}$ & 2.37736 & -0.60765 & -0.11620 \\
\hline $\mathrm{H}$ & -5.83201 & 2.19875 & 0.59252 & $\mathrm{C}$ & 3.59245 & -2.93049 & 1.43018 \\
\hline $\mathrm{H}$ & -5.46709 & 0.46605 & 0.40166 & $\mathrm{H}$ & 2.62305 & -3.44901 & 1.43184 \\
\hline $\mathrm{C}$ & 3.15637 & 3.23380 & 1.50924 & $\mathrm{H}$ & 3.70493 & -2.39209 & 2.38199 \\
\hline $\mathrm{H}$ & 3.37706 & 4.21613 & 1.93013 & $\mathrm{H}$ & 4.39043 & -3.68773 & 1.37521 \\
\hline $\mathrm{C}$ & -3.90774 & 1.55669 & 2.37529 & $\mathrm{C}$ & 5.31314 & -0.79388 & 0.08483 \\
\hline $\mathrm{H}$ & -4.36097 & 0.59192 & 2.63051 & $\mathrm{H}$ & 6.17301 & -1.47923 & 0.13933 \\
\hline $\mathrm{H}$ & -4.56909 & 2.35000 & 2.75130 & $\mathrm{H}$ & 5.31821 & -0.16180 & 0.98597 \\
\hline $\mathrm{H}$ & -2.94225 & 1.63801 & 2.89237 & $\mathrm{H}$ & 5.45265 & -0.13889 & -0.78801 \\
\hline $\mathrm{C}$ & 2.69891 & 2.20656 & 2.32589 & $\mathrm{C}$ & 3.74407 & -2.82376 & -1.61657 \\
\hline $\mathrm{H}$ & 2.55062 & 2.39270 & 3.39250 & $\mathrm{H}$ & 3.86368 & -2.20277 & -2.51564 \\
\hline $\mathrm{C}$ & -2.96214 & -0.55320 & -0.16660 & $\mathrm{H}$ & 2.81180 & -3.39575 & -1.72691 \\
\hline $\mathrm{C}$ & -2.96092 & -1.67071 & 0.68408 & $\mathrm{H}$ & 4.58549 & -3.53224 & -1.56009 \\
\hline $\mathrm{C}$ & 2.60954 & 0.69626 & 0.42766 & $\mathrm{C}$ & 0.41184 & 0.25314 & 3.14984 \\
\hline $\mathrm{C}$ & -3.16650 & -2.92700 & 0.10328 & $\mathrm{H}$ & -0.00756 & -0.49955 & 3.83322 \\
\hline $\mathrm{H}$ & -3.15876 & -3.81283 & 0.74047 & $\mathrm{H}$ & -0.24677 & 0.32323 & 2.26718 \\
\hline $\mathrm{C}$ & -3.15940 & -0.66971 & -1.55983 & $\mathrm{H}$ & 0.39931 & 1.22782 & 3.66319 \\
\hline $\mathrm{C}$ & -3.33464 & -1.95032 & -2.08754 & $\mathrm{~N}$ & -2.68718 & 0.78618 & 0.32364 \\
\hline $\mathrm{H}$ & -3.46128 & -2.07178 & -3.16423 & $\mathrm{Cl}$ & 0.35877 & -2.91335 & -0.20551 \\
\hline $\mathrm{C}$ & -3.15678 & 0.52670 & -2.50472 & $\mathrm{Cl}$ & 1.06577 & -1.21015 & -2.88768 \\
\hline $\mathrm{H}$ & -3.26853 & 1.44124 & -1.90575 & & & & \\
\hline $\mathrm{C}$ & -1.61514 & -2.47938 & 2.67526 & II: & & & \\
\hline $\mathrm{H}$ & -0.66408 & -2.20949 & 2.19959 & 99 & & & \\
\hline $\mathrm{H}$ & -1.80629 & -3.53842 & 2.44673 & & 2977.5786 & & \\
\hline $\mathrm{H}$ & -1.51040 & -2.38816 & 3.76699 & $\mathrm{C}$ & 2.41440 & -1.12034 & 0.78823 \\
\hline $\mathrm{C}$ & -4.06154 & -1.96410 & 2.91488 & $\mathrm{H}$ & 2.60223 & -0.85985 & 1.83629 \\
\hline $\mathrm{H}$ & -4.27551 & -3.03568 & 2.78368 & $\mathrm{Si}$ & -1.28670 & 1.03182 & -0.25099 \\
\hline $\mathrm{H}$ & -4.92970 & -1.41107 & 2.52730 & $\mathrm{C}$ & 1.60197 & -2.27997 & 0.31176 \\
\hline $\mathrm{H}$ & -3.97451 & -1.76880 & 3.99424 & $\mathrm{Si}$ & -4.48759 & 1.33398 & -0.33066 \\
\hline $\mathrm{C}$ & -2.76242 & -1.59007 & 2.18798 & $\mathrm{C}$ & 2.60293 & -0.95108 & -1.53993 \\
\hline
\end{tabular}




\begin{tabular}{|c|c|c|c|}
\hline $\mathrm{C}$ & 1.46497 & -1.93287 & -1.18629 \\
\hline $\mathrm{H}$ & 1.48972 & -2.82719 & -1.82415 \\
\hline $\mathrm{H}$ & 0.50139 & -1.42651 & -1.33021 \\
\hline $\mathrm{C}$ & 0.25783 & -2.41204 & 1.02652 \\
\hline $\mathrm{H}$ & 0.39296 & -2.61888 & 2.09899 \\
\hline $\mathrm{H}$ & -0.31059 & -3.24495 & 0.58646 \\
\hline $\mathrm{H}$ & -0.33978 & -1.49816 & 0.90805 \\
\hline $\mathrm{C}$ & 2.44839 & -3.54588 & 0.56936 \\
\hline $\mathrm{H}$ & 2.64629 & -3.67922 & 1.64322 \\
\hline $\mathrm{H}$ & 3.40869 & -3.53096 & 0.03554 \\
\hline $\mathrm{H}$ & 1.87544 & -4.41690 & 0.22185 \\
\hline $\mathrm{C}$ & -3.23300 & -3.29379 & -0.03252 \\
\hline $\mathrm{H}$ & -3.35515 & -4.13070 & -0.72342 \\
\hline $\mathrm{C}$ & 2.12623 & 0.22106 & -2.38818 \\
\hline $\mathrm{H}$ & 1.23353 & 0.70838 & -1.97564 \\
\hline $\mathrm{H}$ & 1.84561 & -0.17232 & -3.37574 \\
\hline $\mathrm{H}$ & 2.92732 & 0.96190 & -2.53027 \\
\hline $\mathrm{C}$ & -3.11700 & -3.54639 & 1.33235 \\
\hline $\mathrm{H}$ & -3.14802 & -4.57130 & 1.70690 \\
\hline $\mathrm{C}$ & 3.80490 & -1.61163 & -2.21504 \\
\hline $\mathrm{H}$ & 4.65201 & -0.91328 & -2.27829 \\
\hline $\mathrm{H}$ & 3.50913 & -1.87621 & -3.23894 \\
\hline $\mathrm{H}$ & 4.13102 & -2.53103 & -1.71217 \\
\hline $\mathrm{C}$ & -2.97053 & -2.48102 & 2.21331 \\
\hline $\mathrm{H}$ & -2.88345 & -2.67691 & 3.28505 \\
\hline $\mathrm{C}$ & 4.00194 & 0.56074 & 0.06265 \\
\hline $\mathrm{C}$ & 5.34339 & 0.15966 & 0.22596 \\
\hline $\mathrm{C}$ & -3.04655 & -0.90484 & 0.36655 \\
\hline $\mathrm{C}$ & 6.30441 & 1.16902 & 0.32028 \\
\hline $\mathrm{H}$ & 7.35330 & 0.89687 & 0.44733 \\
\hline $\mathrm{C}$ & 3.59678 & 1.90609 & 0.08347 \\
\hline $\mathrm{C}$ & 4.60495 & 2.87215 & 0.17528 \\
\hline $\mathrm{H}$ & 4.32208 & 3.92553 & 0.18921 \\
\hline $\mathrm{C}$ & 2.14170 & 2.32100 & 0.16504 \\
\hline $\mathrm{H}$ & 1.50572 & 1.46246 & -0.09025 \\
\hline $\mathrm{C}$ & 6.03726 & -1.55689 & 1.90345 \\
\hline $\mathrm{H}$ & 5.16075 & -1.31970 & 2.52449 \\
\hline $\mathrm{H}$ & 6.87215 & -0.93630 & 2.26154 \\
\hline $\mathrm{H}$ & 6.30105 & -2.61241 & 2.06478 \\
\hline $\mathrm{C}$ & 6.97089 & -1.68238 & -0.43724 \\
\hline $\mathrm{H}$ & 7.87782 & -1.14674 & -0.12156 \\
\hline $\mathrm{H}$ & 6.80346 & -1.46540 & -1.50147 \\
\hline $\mathrm{H}$ & 7.17406 & -2.75781 & -0.33216 \\
\hline $\mathrm{C}$ & 5.76420 & -1.28937 & 0.41728 \\
\hline $\mathrm{H}$ & 4.92930 & -1.93562 & 0.12146 \\
\hline $\mathrm{C}$ & 1.81971 & 2.67701 & 1.62405 \\
\hline $\mathrm{H}$ & 0.75031 & 2.91066 & 1.71743 \\
\hline $\mathrm{H}$ & 2.40688 & 3.54919 & 1.95216 \\
\hline $\mathrm{H}$ & 2.05602 & 1.84083 & 2.30142 \\
\hline $\mathrm{C}$ & 1.77332 & 3.46346 & -0.78150 \\
\hline $\mathrm{H}$ & 0.69096 & 3.64475 & -0.72409 \\
\hline $\mathrm{H}$ & 2.02147 & 3.22017 & -1.82476 \\
\hline $\mathrm{H}$ & 2.28878 & 4.39729 & -0.50845 \\
\hline $\mathrm{C}$ & -3.19997 & -1.98910 & -0.53381 \\
\hline $\mathrm{C}$ & 5.94333 & 2.51223 & 0.27114 \\
\hline $\mathrm{H}$ & 6.71175 & 3.28374 & 0.33717 \\
\hline $\mathrm{C}$ & -3.29588 & -1.75200 & -2.03307 \\
\hline $\mathrm{H}$ & -3.60157 & -0.70746 & -2.17809 \\
\hline $\mathrm{C}$ & -2.93922 & -1.15809 & 1.75612 \\
\hline
\end{tabular}

\begin{tabular}{|c|c|c|c|}
\hline $\mathrm{C}$ & -2.76658 & -0.03259 & 2.76591 \\
\hline $\mathrm{H}$ & -2.92277 & 0.91636 & 2.23640 \\
\hline $\mathrm{C}$ & -1.92353 & -1.89395 & -2.69909 \\
\hline $\mathrm{H}$ & -1.47687 & -2.87648 & -2.47173 \\
\hline $\mathrm{H}$ & -1.24812 & -1.09689 & -2.35771 \\
\hline $\mathrm{H}$ & -2.01286 & -1.79671 & -3.79201 \\
\hline $\mathrm{C}$ & -4.32556 & -2.65697 & -2.71252 \\
\hline $\mathrm{H}$ & -5.29233 & -2.62709 & -2.18946 \\
\hline $\mathrm{H}$ & -3.98978 & -3.70513 & -2.74684 \\
\hline $\mathrm{H}$ & -4.48450 & -2.33357 & -3.75188 \\
\hline $\mathrm{C}$ & -3.79319 & -0.12239 & 3.89937 \\
\hline $\mathrm{H}$ & -4.81509 & -0.22991 & 3.50810 \\
\hline $\mathrm{H}$ & -3.75561 & 0.78463 & 4.52091 \\
\hline $\mathrm{H}$ & -3.59517 & -0.98364 & 4.55673 \\
\hline $\mathrm{N}$ & -2.97110 & 0.42716 & -0.13606 \\
\hline $\mathrm{C}$ & -5.02652 & 2.18249 & 1.26567 \\
\hline $\mathrm{H}$ & -4.25058 & 2.87632 & 1.61828 \\
\hline $\mathrm{H}$ & -5.24019 & 1.45479 & 2.06234 \\
\hline $\mathrm{H}$ & -5.94897 & 2.75479 & 1.07761 \\
\hline $\mathrm{C}$ & -5.86799 & 0.12198 & -0.77697 \\
\hline $\mathrm{H}$ & -6.83653 & 0.64036 & -0.69704 \\
\hline $\mathrm{H}$ & -5.88982 & -0.74072 & -0.09287 \\
\hline $\mathrm{H}$ & -5.77532 & -0.25661 & -1.80541 \\
\hline $\mathrm{C}$ & -4.33788 & 2.61840 & -1.68752 \\
\hline $\mathrm{H}$ & -4.09063 & 2.15416 & -2.65250 \\
\hline $\mathrm{H}$ & -3.54776 & 3.34566 & -1.45554 \\
\hline $\mathrm{H}$ & -5.29777 & 3.15078 & -1.78111 \\
\hline $\mathrm{C}$ & -1.34415 & 0.00325 & 3.33806 \\
\hline $\mathrm{H}$ & -1.24807 & 0.81815 & 4.07165 \\
\hline $\mathrm{H}$ & -0.60490 & 0.17751 & 2.54122 \\
\hline $\mathrm{H}$ & -1.10039 & -0.94547 & 3.84510 \\
\hline $\mathrm{N}$ & 2.99963 & -0.46765 & -0.14695 \\
\hline $\mathrm{Cl}$ & -1.62246 & 3.04888 & 0.63754 \\
\hline $\mathrm{Cl}$ & -1.18659 & 1.73262 & -2.35746 \\
\hline \multicolumn{4}{|l|}{2} \\
\hline \multicolumn{4}{|c|}{99} \\
\hline \multicolumn{4}{|c|}{$E_{e}=-2977.64363529$} \\
\hline $\mathrm{C}$ & 0.79537 & 0.57819 & 0.80395 \\
\hline $\mathrm{H}$ & 0.77997 & 1.46023 & 0.13812 \\
\hline $\mathrm{Si}$ & -0.29632 & -0.65947 & -0.17915 \\
\hline $\mathrm{C}$ & 0.39404 & 1.06019 & 2.24092 \\
\hline $\mathrm{Si}$ & -2.60555 & -1.12471 & -2.15362 \\
\hline $\mathrm{C}$ & 2.66823 & 0.08498 & 2.31631 \\
\hline $\mathrm{C}$ & 1.75315 & 1.13579 & 2.95643 \\
\hline $\mathrm{H}$ & 2.19539 & 2.13208 & 2.79452 \\
\hline $\mathrm{H}$ & 1.64701 & 0.99865 & 4.04315 \\
\hline $\mathrm{C}$ & -0.55660 & 0.11508 & 2.97787 \\
\hline $\mathrm{H}$ & -1.53804 & 0.09093 & 2.48400 \\
\hline $\mathrm{H}$ & -0.71975 & 0.47711 & 4.00441 \\
\hline $\mathrm{H}$ & -0.17229 & -0.91017 & 3.03674 \\
\hline $\mathrm{C}$ & -0.25078 & 2.44645 & 2.20794 \\
\hline $\mathrm{H}$ & -1.23357 & 2.41996 & 1.71801 \\
\hline $\mathrm{H}$ & 0.39116 & 3.17063 & 1.68115 \\
\hline $\mathrm{H}$ & -0.40077 & 2.81214 & 3.23532 \\
\hline $\mathrm{C}$ & -4.44850 & 0.62809 & 1.94537 \\
\hline $\mathrm{H}$ & -5.06249 & 0.16879 & 2.72293 \\
\hline $\mathrm{C}$ & 2.55310 & -1.27841 & 3.02260 \\
\hline $\mathrm{H}$ & 1.60142 & -1.77316 & 2.79310 \\
\hline
\end{tabular}




\begin{tabular}{|c|c|c|c|c|c|c|c|}
\hline & & & & & & & \\
\hline$\overline{\mathrm{H}}$ & 2.63804 & -1.15257 & 4.11245 & $\mathrm{C}$ & -1.99914 & -0.28264 & -3.73322 \\
\hline $\mathrm{H}$ & 3.35840 & -1.95188 & 2.70157 & $\mathrm{H}$ & -1.26878 & -0.92592 & -4.24400 \\
\hline $\mathrm{C}$ & -4.49533 & 2.00495 & 1.76288 & $\mathrm{H}$ & -1.50366 & 0.67698 & -3.53364 \\
\hline $\mathrm{H}$ & -5.12866 & 2.62289 & 2.40103 & $\mathrm{H}$ & -2.84142 & -0.09999 & -4.41780 \\
\hline $\mathrm{C}$ & 4.12436 & 0.54374 & 2.39050 & $\mathrm{C}$ & -4.47894 & -1.00408 & -2.05890 \\
\hline $\mathrm{H}$ & 4.79563 & -0.16047 & 1.88048 & $\mathrm{H}$ & -4.89006 & -1.20681 & -3.06002 \\
\hline $\mathrm{H}$ & 4.42727 & 0.60862 & 3.44598 & $\mathrm{H}$ & -4.84341 & -0.02056 & -1.72853 \\
\hline $\mathrm{H}$ & 4.25442 & 1.53130 & 1.92797 & $\mathrm{H}$ & -4.87925 & -1.76408 & -1.37474 \\
\hline $\mathrm{C}$ & -3.75418 & 2.58487 & 0.73952 & $\mathrm{C}$ & -2.13928 & -2.94375 & -2.21431 \\
\hline $\mathrm{H}$ & -3.82282 & 3.66098 & 0.57150 & $\mathrm{H}$ & -2.48697 & -3.48601 & -1.32288 \\
\hline $\mathrm{C}$ & 3.07850 & 0.10479 & -0.18607 & $\mathrm{H}$ & -1.05350 & -3.08921 & -2.30261 \\
\hline $\mathrm{C}$ & 3.41256 & 1.33727 & -0.79926 & $\mathrm{H}$ & -2.61538 & -3.39552 & -3.09916 \\
\hline $\mathrm{C}$ & -2.83073 & 0.42022 & 0.15707 & $\mathrm{C}$ & -1.60841 & 3.84972 & -0.91853 \\
\hline $\mathrm{C}$ & 4.24665 & 1.34056 & -1.92179 & $\mathrm{H}$ & -1.04421 & 4.22476 & -1.78494 \\
\hline $\mathrm{H}$ & 4.48266 & 2.28993 & -2.40780 & $\mathrm{H}$ & -0.92524 & 3.80713 & -0.06055 \\
\hline $\mathrm{C}$ & 3.66142 & -1.09100 & -0.67480 & $\mathrm{H}$ & -2.39203 & 4.58978 & -0.69758 \\
\hline $\mathrm{C}$ & 4.51962 & -1.03466 & -1.77597 & $\mathrm{~N}$ & 2.16551 & 0.04396 & 0.91506 \\
\hline $\mathrm{H}$ & 4.96262 & -1.95979 & -2.15126 & $\mathrm{Cl}$ & 0.68532 & -0.79862 & -2.02039 \\
\hline $\mathrm{C}$ & 3.39155 & -2.44962 & -0.04931 & $\mathrm{Cl}$ & -0.12815 & -2.56638 & 0.66783 \\
\hline $\mathrm{H}$ & 2.60983 & -2.30864 & 0.70543 & & & & \\
\hline $\mathrm{C}$ & 1.92480 & 3.31671 & -1.27468 & III & & & \\
\hline $\mathrm{H}$ & 1.10364 & 2.63010 & -1.53147 & 15 & & & \\
\hline $\mathrm{H}$ & 2.43544 & 3.57539 & -2.21512 & & 3813.0076 & & \\
\hline $\mathrm{H}$ & 1.49293 & 4.24040 & -0.85985 & $\mathrm{C}$ & -3.02012 & -1.55489 & -0.80645 \\
\hline $\mathrm{C}$ & 4.06914 & 3.65584 & -0.01277 & $\mathrm{H}$ & -2.22078 & -0.79821 & -0.84921 \\
\hline $\mathrm{H}$ & 4.58912 & 3.93728 & -0.94009 & $\mathrm{Si}$ & 2.13194 & -0.57641 & -0.45981 \\
\hline $\mathrm{H}$ & 4.81440 & 3.21815 & 0.66634 & $\mathrm{C}$ & -3.13614 & -2.71739 & -1.75148 \\
\hline $\mathrm{H}$ & 3.68510 & 4.58015 & 0.44448 & $\mathrm{Si}$ & 1.39801 & -2.90935 & 1.67118 \\
\hline $\mathrm{C}$ & 2.91682 & 2.68337 & -0.29093 & $\mathrm{C}$ & -4.91423 & -2.67002 & -0.03242 \\
\hline $\mathrm{H}$ & 2.40556 & 2.51520 & 0.66632 & $\mathrm{C}$ & -4.56233 & -3.23002 & -1.42358 \\
\hline $\mathrm{C}$ & 2.87204 & -3.47911 & -1.05836 & $\mathrm{H}$ & -5.27948 & -2.84734 & -2.16660 \\
\hline $\mathrm{H}$ & 2.62650 & -4.41822 & -0.53977 & $\mathrm{H}$ & -4.61928 & -4.32638 & -1.43638 \\
\hline $\mathrm{H}$ & 3.62839 & -3.71050 & -1.82437 & $\mathrm{C}$ & -2.05145 & -3.79412 & -1.60481 \\
\hline $\mathrm{H}$ & 1.96697 & -3.12186 & -1.56532 & $\mathrm{H}$ & -1.05381 & -3.34030 & -1.68542 \\
\hline $\mathrm{C}$ & 4.65122 & -2.99255 & 0.63781 & $\mathrm{H}$ & -2.18388 & -4.51829 & -2.42177 \\
\hline $\mathrm{H}$ & 4.42698 & -3.92635 & 1.17592 & $\mathrm{H}$ & -2.12805 & -4.33728 & -0.65444 \\
\hline $\mathrm{H}$ & 5.07319 & -2.27521 & 1.35619 & $\mathrm{C}$ & -3.02474 & -2.11811 & -3.16710 \\
\hline $\mathrm{H}$ & 5.43428 & -3.21182 & -0.10486 & $\mathrm{H}$ & -2.01671 & -1.70228 & -3.31446 \\
\hline $\mathrm{C}$ & -3.64700 & -0.18923 & 1.14082 & $\mathrm{H}$ & -3.76895 & -1.32646 & -3.33833 \\
\hline $\mathrm{C}$ & 4.79933 & 0.16628 & -2.41562 & $\mathrm{H}$ & -3.19595 & -2.91690 & -3.90317 \\
\hline $\mathrm{H}$ & 5.45421 & 0.18892 & -3.28805 & $\mathrm{C}$ & 4.20803 & -4.68402 & -1.72170 \\
\hline $\mathrm{C}$ & -3.74211 & -1.69951 & 1.32895 & $\mathrm{H}$ & 4.03734 & -5.39538 & -2.53283 \\
\hline $\mathrm{H}$ & -3.19996 & -2.17316 & 0.49753 & $\mathrm{C}$ & -4.47156 & -3.61166 & 1.08888 \\
\hline $\mathrm{C}$ & -2.91880 & 1.81456 & -0.07562 & $\mathrm{H}$ & -3.39292 & -3.81755 & 1.03736 \\
\hline $\mathrm{C}$ & -2.20393 & 2.47856 & -1.24435 & $\mathrm{H}$ & -5.01881 & -4.55941 & 0.99047 \\
\hline $\mathrm{H}$ & -1.38127 & 1.81598 & -1.55533 & $\mathrm{H}$ & -4.69643 & -3.18771 & 2.07540 \\
\hline $\mathrm{C}$ & -3.10591 & -2.19435 & 2.63389 & $\mathrm{C}$ & 5.48434 & -4.55661 & -1.18188 \\
\hline $\mathrm{H}$ & -3.51942 & -1.65076 & 3.49833 & $\mathrm{H}$ & 6.30599 & -5.16711 & -1.56029 \\
\hline $\mathrm{H}$ & -2.01792 & -2.07551 & 2.63419 & $\mathrm{C}$ & -6.37957 & -2.29904 & 0.13712 \\
\hline $\mathrm{H}$ & -3.32431 & -3.26394 & 2.77129 & $\mathrm{H}$ & -6.55980 & -1.83929 & 1.11913 \\
\hline $\mathrm{C}$ & -5.20696 & -2.16298 & 1.29233 & $\mathrm{H}$ & -6.97658 & -3.21941 & 0.07351 \\
\hline $\mathrm{H}$ & -5.77245 & -1.69854 & 0.47386 & $\mathrm{H}$ & -6.72856 & -1.61087 & -0.64369 \\
\hline $\mathrm{H}$ & -5.72008 & -1.91140 & 2.23300 & $\mathrm{C}$ & 5.70386 & -3.63718 & -0.16362 \\
\hline $\mathrm{H}$ & -5.25561 & -3.25592 & 1.17772 & $\mathrm{H}$ & 6.70804 & -3.52479 & 0.25281 \\
\hline $\mathrm{C}$ & -3.17831 & 2.61365 & -2.42332 & $\mathrm{C}$ & -4.36011 & -0.21545 & 0.69179 \\
\hline $\mathrm{H}$ & -3.62615 & 1.65016 & -2.70066 & $\mathrm{C}$ & -5.06718 & 0.73953 & -0.07164 \\
\hline $\mathrm{H}$ & -2.66744 & 3.02078 & -3.30898 & $\mathrm{C}$ & 3.36507 & -2.98756 & -0.20146 \\
\hline $\mathrm{H}$ & -3.99835 & 3.29724 & -2.15316 & $\mathrm{C}$ & -5.43734 & 1.92367 & 0.56577 \\
\hline $\mathrm{N}$ & -1.94025 & -0.37298 & -0.64825 & $\mathrm{H}$ & -5.97196 & 2.69071 & 0.00425 \\
\hline
\end{tabular}




\begin{tabular}{|c|c|c|c|c|c|c|c|}
\hline & & & & & & & \\
\hline $\mathrm{C}$ & -4.00723 & -0.00846 & 2.03302 & $\mathrm{H}$ & 6.85395 & -1.13564 & 0.57507 \\
\hline $\mathrm{C}$ & -4.41813 & 1.19405 & 2.62249 & $\mathrm{~N}$ & -4.01830 & -1.44286 & -0.00332 \\
\hline $\mathrm{H}$ & -4.15374 & 1.38681 & 3.66364 & $\mathrm{Cl}$ & 1.20860 & 0.42942 & 1.31067 \\
\hline $\mathrm{C}$ & -3.17296 & -0.97669 & 2.84965 & $\mathrm{Cl}$ & 0.00374 & -0.88686 & -1.53482 \\
\hline $\mathrm{H}$ & -2.89830 & -1.82025 & 2.20269 & $\mathrm{C}$ & 1.48697 & 2.64534 & -1.52972 \\
\hline $\mathrm{C}$ & -4.31172 & 1.26569 & -2.41898 & $\mathrm{C}$ & 1.67077 & 2.92098 & -3.00981 \\
\hline $\mathrm{H}$ & -3.29967 & 0.86487 & -2.25822 & $\mathrm{C}$ & 1.34209 & 4.42477 & -3.20861 \\
\hline $\mathrm{H}$ & -4.27933 & 2.34064 & -2.18405 & $\mathrm{C}$ & 1.41940 & 5.06235 & -1.81008 \\
\hline $\mathrm{H}$ & -4.55729 & 1.15497 & -3.48579 & $\mathrm{H}$ & 0.32043 & 4.53115 & -3.60564 \\
\hline $\mathrm{C}$ & -6.77386 & 1.03492 & -1.93067 & $\mathrm{~N}$ & 1.37505 & 3.81338 & -0.94930 \\
\hline $\mathrm{H}$ & -6.86043 & 2.12916 & -1.87004 & $\mathrm{C}$ & 1.18879 & 3.88712 & 0.47882 \\
\hline $\mathrm{H}$ & -7.53975 & 0.59850 & -1.27293 & $\mathrm{C}$ & -0.11990 & 3.79864 & 0.98758 \\
\hline $\mathrm{H}$ & -7.00144 & 0.74955 & -2.96750 & $\mathrm{C}$ & 2.30549 & 3.94028 & 1.33103 \\
\hline $\mathrm{C}$ & -5.36717 & 0.56122 & -1.55549 & $\mathrm{C}$ & -0.29642 & 3.83300 & 2.37285 \\
\hline $\mathrm{H}$ & -5.31805 & -0.51127 & -1.79342 & $\mathrm{C}$ & 2.07940 & 3.97691 & 2.71082 \\
\hline $\mathrm{C}$ & -1.86599 & -0.32323 & 3.31667 & $\mathrm{C}$ & 0.79212 & 3.93708 & 3.23193 \\
\hline $\mathrm{H}$ & -1.24670 & -1.06073 & 3.84879 & $\mathrm{H}$ & -1.30413 & 3.74911 & 2.78623 \\
\hline $\mathrm{H}$ & -2.06712 & 0.50862 & 4.00959 & $\mathrm{H}$ & 2.93372 & 4.00723 & 3.38971 \\
\hline $\mathrm{H}$ & -1.26527 & 0.06468 & 2.48168 & $\mathrm{H}$ & 0.63849 & 3.95830 & 4.31210 \\
\hline $\mathrm{C}$ & -3.96340 & -1.51322 & 4.04873 & $\mathrm{C}$ & 3.13385 & 2.58332 & -3.33423 \\
\hline $\mathrm{H}$ & -3.39021 & -2.30028 & 4.56006 & $\mathrm{H}$ & 3.34281 & 1.53801 & -3.06507 \\
\hline $\mathrm{H}$ & -4.94055 & -1.92656 & 3.75676 & $\mathrm{H}$ & 3.32926 & 2.72743 & -4.40857 \\
\hline $\mathrm{H}$ & -4.15269 & -0.71241 & 4.77940 & $\mathrm{H}$ & 3.83155 & 3.21956 & -2.76820 \\
\hline $\mathrm{C}$ & 3.14303 & -3.91230 & -1.25125 & $\mathrm{C}$ & 0.74570 & 2.00743 & -3.81473 \\
\hline $\mathrm{C}$ & -5.12524 & 2.14742 & 1.90436 & $\mathrm{H}$ & 0.96133 & 0.95464 & -3.58425 \\
\hline $\mathrm{H}$ & -5.42505 & 3.08060 & 2.38327 & $\mathrm{H}$ & -0.30821 & 2.18933 & -3.54899 \\
\hline $\mathrm{C}$ & 1.78010 & -4.03130 & -1.91137 & $\mathrm{H}$ & 0.86806 & 2.18407 & -4.89590 \\
\hline $\mathrm{H}$ & 1.05724 & -3.54745 & -1.23741 & $\mathrm{C}$ & 3.73081 & 3.83099 & 0.81784 \\
\hline $\mathrm{C}$ & 4.66463 & -2.84517 & 0.33754 & $\mathrm{H}$ & 3.71114 & 3.97537 & -0.26722 \\
\hline $\mathrm{C}$ & 4.97311 & -1.83750 & 1.43287 & $\mathrm{C}$ & -1.31804 & 3.53206 & 0.09279 \\
\hline $\mathrm{H}$ & 4.02514 & -1.36957 & 1.73357 & $\mathrm{H}$ & -1.01459 & 3.70019 & -0.94715 \\
\hline $\mathrm{C}$ & 1.76745 & -3.24971 & -3.23045 & $\mathrm{C}$ & 2.71809 & 5.84064 & -1.58525 \\
\hline $\mathrm{H}$ & 2.47156 & -3.70329 & -3.94675 & $\mathrm{H}$ & 2.69611 & 6.75879 & -2.19001 \\
\hline $\mathrm{H}$ & 2.05803 & -2.20183 & -3.07285 & $\mathrm{H}$ & 2.82485 & 6.13003 & -0.52917 \\
\hline $\mathrm{H}$ & 0.76051 & -3.25380 & -3.67874 & $\mathrm{H}$ & 3.60064 & 5.26037 & -1.88436 \\
\hline $\mathrm{C}$ & 1.34412 & -5.47935 & -2.14756 & $\mathrm{C}$ & 0.24630 & 5.99561 & -1.50995 \\
\hline $\mathrm{H}$ & 1.39921 & -6.08112 & -1.22875 & $\mathrm{H}$ & 0.24763 & 6.30803 & -0.45450 \\
\hline $\mathrm{H}$ & 1.97036 & -5.97183 & -2.90709 & $\mathrm{H}$ & 0.33769 & 6.89713 & -2.13314 \\
\hline $\mathrm{H}$ & 0.30770 & -5.50841 & -2.51882 & $\mathrm{H}$ & -0.72040 & 5.52872 & -1.73765 \\
\hline $\mathrm{C}$ & 5.58445 & -2.50893 & 2.66641 & $\mathrm{C}$ & -1.71287 & 2.05683 & 0.19903 \\
\hline $\mathrm{H}$ & 4.95732 & -3.33998 & 3.02260 & $\mathrm{H}$ & -2.59428 & 1.86210 & -0.42989 \\
\hline $\mathrm{H}$ & 5.69472 & -1.78316 & 3.48655 & $\mathrm{H}$ & -0.88154 & 1.40753 & -0.11537 \\
\hline $\mathrm{H}$ & 6.58310 & -2.91743 & 2.44616 & $\mathrm{H}$ & -1.97647 & 1.80757 & 1.23892 \\
\hline $\mathrm{N}$ & 2.28249 & -2.20858 & 0.31680 & $\mathrm{C}$ & -2.50305 & 4.45026 & 0.39563 \\
\hline $\mathrm{C}$ & 1.86587 & -2.24053 & 3.37073 & $\mathrm{H}$ & -3.31808 & 4.26944 & -0.32342 \\
\hline $\mathrm{H}$ & 1.68822 & -1.15934 & 3.43397 & $\mathrm{H}$ & -2.91061 & 4.26120 & 1.40074 \\
\hline $\mathrm{H}$ & 2.92658 & -2.43270 & 3.59085 & $\mathrm{H}$ & -2.21968 & 5.51190 & 0.33895 \\
\hline $\mathrm{H}$ & 1.26737 & -2.75291 & 4.14200 & $\mathrm{C}$ & 4.26809 & 2.41640 & 1.05502 \\
\hline $\mathrm{C}$ & 1.75169 & -4.76356 & 1.71024 & $\mathrm{H}$ & 3.64780 & 1.67785 & 0.52801 \\
\hline $\mathrm{H}$ & 2.82550 & -4.96198 & 1.84967 & $\mathrm{H}$ & 5.30294 & 2.33562 & 0.68854 \\
\hline $\mathrm{H}$ & 1.44633 & -5.24974 & 0.77183 & $\mathrm{H}$ & 4.25731 & 2.16269 & 2.12707 \\
\hline $\mathrm{H}$ & 1.20307 & -5.23551 & 2.54083 & $\mathrm{C}$ & 4.65953 & 4.88737 & 1.42054 \\
\hline $\mathrm{C}$ & -0.46334 & -2.70704 & 1.41247 & $\mathrm{H}$ & 4.80741 & 4.72400 & 2.49874 \\
\hline $\mathrm{H}$ & -0.75728 & -3.29608 & 0.53387 & $\mathrm{H}$ & 5.65025 & 4.83694 & 0.94509 \\
\hline $\mathrm{H}$ & -0.70811 & -1.65175 & 1.22857 & $\mathrm{H}$ & 4.26421 & 5.90552 & 1.28722 \\
\hline $\mathrm{H}$ & -1.00983 & -3.07667 & 2.29592 & $\mathrm{H}$ & 2.02292 & 4.92041 & -3.91685 \\
\hline $\mathrm{C}$ & 5.88534 & -0.72630 & 0.90370 & & & & \\
\hline $\mathrm{H}$ & 6.07828 & 0.02335 & 1.68632 & \multirow{2}{*}{\multicolumn{4}{|c|}{$\begin{array}{l}\text { [IIIa-IVa] } \\
151\end{array}$}} \\
\hline $\mathrm{H}$ & 5.41870 & -0.21962 & 0.04625 & & & & \\
\hline
\end{tabular}




\begin{tabular}{|c|c|c|c|}
\hline \multicolumn{4}{|c|}{$\mathrm{E}_{\mathrm{e}}=-3812.97086173$} \\
\hline $\mathrm{C}$ & -3.39136 & -0.43773 & -1.19826 \\
\hline $\mathrm{H}$ & -3.23703 & 0.63349 & -1.03688 \\
\hline $\mathrm{Si}$ & 1.30076 & -0.46107 & 0.32739 \\
\hline $\mathrm{C}$ & -3.56294 & -0.97741 & -2.63236 \\
\hline $\mathrm{Si}$ & 3.02977 & -2.27354 & 2.38589 \\
\hline $\mathrm{C}$ & -4.78961 & -2.32951 & -0.93988 \\
\hline $\mathrm{C}$ & -4.74813 & -1.98014 & -2.45068 \\
\hline $\mathrm{H}$ & -5.70012 & -1.48822 & -2.72774 \\
\hline $\mathrm{H}$ & -4.63766 & -2.88012 & -3.08127 \\
\hline $\mathrm{C}$ & -2.33633 & -1.68132 & -3.24776 \\
\hline $\mathrm{H}$ & -1.49042 & -0.98191 & -3.35256 \\
\hline $\mathrm{H}$ & -2.60819 & -2.05946 & -4.25275 \\
\hline $\mathrm{H}$ & -1.99465 & -2.52586 & -2.63064 \\
\hline $\mathrm{C}$ & -3.95190 & 0.19984 & -3.55742 \\
\hline $\mathrm{H}$ & -3.11717 & 0.92429 & -3.62736 \\
\hline $\mathrm{H}$ & -4.84460 & 0.73123 & -3.18294 \\
\hline $\mathrm{H}$ & -4.16945 & -0.17204 & -4.57794 \\
\hline $\mathrm{C}$ & 3.49746 & -3.72091 & -2.22228 \\
\hline $\mathrm{H}$ & 3.02938 & -4.51855 & -2.81337 \\
\hline $\mathrm{C}$ & -3.83724 & -3.50446 & -0.62198 \\
\hline $\mathrm{H}$ & -2.78858 & -3.21239 & -0.80094 \\
\hline $\mathrm{H}$ & -4.08724 & -4.36664 & -1.26863 \\
\hline $\mathrm{H}$ & -3.93501 & -3.82219 & 0.42833 \\
\hline $\mathrm{C}$ & 4.77193 & -3.25771 & -2.56720 \\
\hline $\mathrm{H}$ & 5.30100 & -3.67784 & -3.43253 \\
\hline $\mathrm{C}$ & -6.20496 & -2.63953 & -0.44340 \\
\hline $\mathrm{H}$ & -6.21137 & -2.79390 & 0.65096 \\
\hline $\mathrm{H}$ & -6.56647 & -3.56455 & -0.92946 \\
\hline $\mathrm{H}$ & -6.90060 & -1.81685 & -0.68470 \\
\hline $\mathrm{C}$ & 5.37721 & -2.27062 & -1.77759 \\
\hline $\mathrm{H}$ & 6.39219 & -1.93134 & -2.02017 \\
\hline $\mathrm{C}$ & -4.78033 & -0.38881 & 0.81214 \\
\hline $\mathrm{C}$ & -5.75926 & 0.63251 & 0.65003 \\
\hline $\mathrm{C}$ & 3.39495 & -2.15023 & -0.34859 \\
\hline $\mathrm{C}$ & -6.18774 & 1.34192 & 1.78718 \\
\hline $\mathrm{H}$ & -6.93441 & 2.13756 & 1.67147 \\
\hline $\mathrm{C}$ & -4.27152 & -0.71536 & 2.09584 \\
\hline $\mathrm{C}$ & -4.74604 & 0.01089 & 3.20668 \\
\hline $\mathrm{H}$ & -4.35572 & -0.22943 & 4.20270 \\
\hline $\mathrm{C}$ & -3.26365 & -1.83509 & 2.31256 \\
\hline $\mathrm{H}$ & -2.86809 & -2.12488 & 1.32676 \\
\hline $\mathrm{C}$ & -5.85518 & 2.35150 & -1.20635 \\
\hline $\mathrm{H}$ & -4.75434 & 2.35771 & -1.29114 \\
\hline $\mathrm{H}$ & -6.14832 & 3.15636 & -0.50516 \\
\hline $\mathrm{H}$ & -6.28110 & 2.58721 & -2.20121 \\
\hline $\mathrm{C}$ & -7.89922 & 0.93396 & -0.69376 \\
\hline $\mathrm{H}$ & -8.32023 & 1.71475 & -0.03192 \\
\hline $\mathrm{H}$ & -8.26559 & -0.04686 & -0.33562 \\
\hline $\mathrm{H}$ & -8.29704 & 1.10602 & -1.71288 \\
\hline $\mathrm{C}$ & -6.35761 & 0.98124 & -0.70937 \\
\hline $\mathrm{H}$ & -6.01596 & 0.22223 & -1.43097 \\
\hline $\mathrm{C}$ & -2.05867 & -1.39865 & 3.16338 \\
\hline $\mathrm{H}$ & -1.35249 & -2.24228 & 3.26353 \\
\hline $\mathrm{H}$ & -2.36595 & -1.09781 & 4.18394 \\
\hline $\mathrm{H}$ & -1.52123 & -0.55933 & 2.69297 \\
\hline $\mathrm{C}$ & -3.95933 & -3.05611 & 2.95429 \\
\hline $\mathrm{H}$ & -3.25259 & -3.90521 & 3.03424 \\
\hline $\mathrm{H}$ & -4.83689 & -3.38669 & 2.36671 \\
\hline
\end{tabular}

\begin{tabular}{|c|c|c|c|}
\hline $\mathrm{H}$ & -4.31441 & -2.80888 & 3.97406 \\
\hline $\mathrm{C}$ & 2.80020 & -3.20047 & -1.11349 \\
\hline $\mathrm{C}$ & -5.68544 & 1.03807 & 3.05937 \\
\hline $\mathrm{H}$ & -6.03086 & 1.59989 & 3.93691 \\
\hline $\mathrm{C}$ & 1.45747 & -3.83324 & -0.74714 \\
\hline $\mathrm{H}$ & 1.05844 & -3.30094 & 0.13272 \\
\hline $\mathrm{C}$ & 4.71523 & -1.70362 & -0.67215 \\
\hline $\mathrm{C}$ & 5.47148 & -0.70902 & 0.20985 \\
\hline $\mathrm{H}$ & 4.74045 & -0.26586 & 0.90397 \\
\hline $\mathrm{C}$ & 0.42013 & -3.70394 & -1.87867 \\
\hline $\mathrm{H}$ & 0.77022 & -4.21439 & -2.79745 \\
\hline $\mathrm{H}$ & 0.21716 & -2.64785 & -2.11124 \\
\hline $\mathrm{H}$ & -0.53413 & -4.17460 & -1.57203 \\
\hline $\mathrm{C}$ & 1.63486 & -5.31803 & -0.36037 \\
\hline $\mathrm{H}$ & 2.37186 & -5.44046 & 0.45250 \\
\hline $\mathrm{H}$ & 1.98614 & -5.91049 & -1.22795 \\
\hline $\mathrm{H}$ & 0.66804 & -5.74429 & -0.02689 \\
\hline $\mathrm{C}$ & 6.54444 & -1.44797 & 1.04203 \\
\hline $\mathrm{H}$ & 6.11100 & -2.26751 & 1.63853 \\
\hline $\mathrm{H}$ & 7.04520 & -0.74253 & 1.73429 \\
\hline $\mathrm{H}$ & 7.31718 & -1.88269 & 0.37760 \\
\hline $\mathrm{N}$ & 2.69545 & -1.56485 & 0.75999 \\
\hline $\mathrm{C}$ & 3.95802 & -1.08461 & 3.54088 \\
\hline $\mathrm{H}$ & 3.34825 & -0.19366 & 3.77558 \\
\hline $\mathrm{H}$ & 4.91454 & -0.74447 & 3.10407 \\
\hline $\mathrm{H}$ & 4.18774 & -1.60905 & 4.49167 \\
\hline $\mathrm{C}$ & 4.07864 & -3.85371 & 2.15848 \\
\hline $\mathrm{H}$ & 4.67393 & -3.85780 & 1.22792 \\
\hline $\mathrm{H}$ & 3.42772 & -4.74846 & 2.14508 \\
\hline $\mathrm{H}$ & 4.77517 & -3.96309 & 3.01387 \\
\hline $\mathrm{C}$ & 1.40685 & -2.80668 & 3.21197 \\
\hline $\mathrm{H}$ & 0.84141 & -3.49234 & 2.54991 \\
\hline $\mathrm{H}$ & 0.76445 & -1.93969 & 3.44325 \\
\hline $\mathrm{H}$ & 1.61973 & -3.34557 & 4.15814 \\
\hline $\mathrm{C}$ & 6.13896 & 0.44577 & -0.56420 \\
\hline $\mathrm{H}$ & 6.77247 & 1.03876 & 0.12227 \\
\hline $\mathrm{H}$ & 5.39038 & 1.12142 & -1.00452 \\
\hline $\mathrm{H}$ & 6.79325 & 0.07336 & -1.37591 \\
\hline $\mathrm{N}$ & -4.28547 & -1.04324 & -0.36344 \\
\hline $\mathrm{Cl}$ & 1.09055 & 0.60131 & 2.23684 \\
\hline $\mathrm{Cl}$ & -1.11341 & -0.71343 & -0.45403 \\
\hline $\mathrm{C}$ & 1.96915 & 1.26347 & -1.10607 \\
\hline $\mathrm{C}$ & 2.15177 & 0.94618 & -2.59904 \\
\hline $\mathrm{C}$ & 1.42464 & 2.11572 & -3.31882 \\
\hline $\mathrm{C}$ & 1.37419 & 3.27106 & -2.31243 \\
\hline $\mathrm{H}$ & 0.38966 & 1.80805 & -3.56093 \\
\hline $\mathrm{N}$ & 1.43647 & 2.48809 & -0.99423 \\
\hline $\mathrm{C}$ & 1.29100 & 3.23410 & 0.24021 \\
\hline $\mathrm{C}$ & -0.00518 & 3.59349 & 0.71396 \\
\hline $\mathrm{C}$ & 2.46336 & 3.66615 & 0.92346 \\
\hline $\mathrm{C}$ & -0.08808 & 4.46402 & 1.81873 \\
\hline $\mathrm{C}$ & 2.31439 & 4.53424 & 2.02252 \\
\hline $\mathrm{C}$ & 1.05406 & 4.95272 & 2.45964 \\
\hline $\mathrm{H}$ & -1.08013 & 4.74560 & 2.19107 \\
\hline $\mathrm{H}$ & 3.21393 & 4.87466 & 2.54931 \\
\hline $\mathrm{H}$ & 0.96150 & 5.63504 & 3.31445 \\
\hline $\mathrm{C}$ & 3.65353 & 0.89743 & -2.96895 \\
\hline $\mathrm{H}$ & 4.14134 & 0.04795 & -2.46613 \\
\hline $\mathrm{H}$ & 3.74520 & 0.75345 & -4.06286 \\
\hline
\end{tabular}




\begin{tabular}{|c|c|c|c|c|c|c|c|}
\hline & & & & \multirow{2}{*}{\multicolumn{3}{|c|}{-4.36594}} & \multirow[b]{2}{*}{-2.34378} \\
\hline $\mathrm{H}$ & 4.19038 & 1.82220 & -2.69872 & & & & \\
\hline $\mathrm{C}$ & 1.53351 & -0.40322 & -3.01193 & $\mathrm{H}$ & -3.94099 & -3.68494 & -0.62042 \\
\hline $\mathrm{H}$ & 2.07510 & -1.24189 & -2.54601 & $\mathrm{C}$ & 4.00485 & -3.97883 & -2.49850 \\
\hline $\mathrm{H}$ & 0.47512 & -0.45561 & -2.70964 & $\mathrm{H}$ & 4.25571 & -4.65465 & -3.31753 \\
\hline $\mathrm{H}$ & 1.60669 & -0.51580 & -4.11214 & $\mathrm{C}$ & -6.31128 & -2.43813 & -0.77304 \\
\hline $\mathrm{C}$ & 3.87712 & 3.18445 & 0.60012 & $\mathrm{H}$ & -6.14498 & -2.88980 & 0.21598 \\
\hline $\mathrm{H}$ & 3.83669 & 2.53451 & -0.28904 & $\mathrm{H}$ & -6.73475 & -3.20653 & -1.43563 \\
\hline $\mathrm{C}$ & -1.32588 & 3.03367 & 0.17638 & $\mathrm{H}$ & -7.04581 & -1.62816 & -0.66249 \\
\hline $\mathrm{H}$ & -1.10438 & 2.38211 & -0.68677 & $\mathrm{C}$ & 4.82969 & -2.89595 & -2.20667 \\
\hline $\mathrm{C}$ & 2.59606 & 4.20781 & -2.38150 & $\mathrm{H}$ & 5.72340 & -2.73430 & -2.81020 \\
\hline $\mathrm{H}$ & 2.60692 & 4.71839 & -3.36207 & $\mathrm{C}$ & -4.69001 & -0.58562 & 0.85855 \\
\hline $\mathrm{H}$ & 2.53179 & 4.97427 & -1.58881 & $\mathrm{C}$ & -5.67762 & 0.36113 & 1.22448 \\
\hline $\mathrm{H}$ & 3.54462 & 3.66442 & -2.25560 & $\mathrm{C}$ & 3.36289 & -2.23619 & -0.38737 \\
\hline $\mathrm{C}$ & 0.11502 & 4.12082 & -2.47561 & $\mathrm{C}$ & -5.84484 & 0.67533 & 2.57545 \\
\hline $\mathrm{H}$ & 0.05596 & 4.89065 & -1.68823 & $\mathrm{H}$ & -6.59640 & 1.41291 & 2.86556 \\
\hline $\mathrm{H}$ & 0.16755 & 4.63281 & -3.45494 & $\mathrm{C}$ & -3.90650 & -1.22021 & 1.84693 \\
\hline $\mathrm{H}$ & -0.79866 & 3.50629 & -2.45437 & $\mathrm{C}$ & -4.12726 & -0.88917 & 3.18897 \\
\hline $\mathrm{C}$ & -2.02274 & 2.15968 & 1.24616 & $\mathrm{H}$ & -3.52935 & -1.37668 & 3.96128 \\
\hline $\mathrm{H}$ & -2.97413 & 1.75665 & 0.85353 & $\mathrm{C}$ & -2.86178 & -2.27277 & 1.51975 \\
\hline $\mathrm{H}$ & -1.39139 & 1.31446 & 1.55063 & $\mathrm{H}$ & -2.73142 & -2.27962 & 0.43152 \\
\hline $\mathrm{H}$ & -2.26107 & 2.76594 & 2.14093 & $\mathrm{C}$ & -6.26562 & 2.54324 & 0.08997 \\
\hline $\mathrm{C}$ & -2.31802 & 4.14171 & -0.24875 & $\mathrm{H}$ & -5.21704 & 2.72652 & -0.18466 \\
\hline $\mathrm{H}$ & -3.18686 & 3.68850 & -0.76076 & $\mathrm{H}$ & -6.45028 & 3.05193 & 1.04857 \\
\hline $\mathrm{H}$ & -2.70442 & 4.67369 & 0.64161 & $\mathrm{H}$ & -6.90545 & 3.01451 & -0.67159 \\
\hline $\mathrm{H}$ & -1.87516 & 4.89387 & -0.92231 & $\mathrm{C}$ & -8.05584 & 0.82892 & 0.51525 \\
\hline $\mathrm{C}$ & 4.39996 & 2.31895 & 1.76788 & $\mathrm{H}$ & -8.34417 & 1.33892 & 1.44654 \\
\hline $\mathrm{H}$ & 3.74617 & 1.44799 & 1.93352 & $\mathrm{H}$ & -8.29169 & -0.23823 & 0.63414 \\
\hline $\mathrm{H}$ & 5.42360 & 1.96463 & 1.55469 & $\mathrm{H}$ & -8.68209 & 1.23567 & -0.29266 \\
\hline $\mathrm{H}$ & 4.42961 & 2.90842 & 2.70424 & $\mathrm{C}$ & -6.57125 & 1.04580 & 0.20154 \\
\hline $\mathrm{C}$ & 4.85735 & 4.33932 & 0.31078 & $\mathrm{H}$ & -6.36716 & 0.58501 & -0.77515 \\
\hline $\mathrm{H}$ & 5.00947 & 4.96651 & 1.21016 & $\mathrm{C}$ & -1.49269 & -1.95647 & 2.12424 \\
\hline $\mathrm{H}$ & 5.84349 & 3.92889 & 0.01878 & $\mathrm{H}$ & -0.79405 & -2.78227 & 1.91973 \\
\hline $\mathrm{H}$ & 4.49659 & 4.99301 & -0.50372 & $\mathrm{H}$ & -1.53952 & -1.83285 & 3.21725 \\
\hline \multirow[t]{2}{*}{$\mathrm{H}$} & 1.92250 & 2.41012 & -4.26002 & $\mathrm{H}$ & -1.06994 & -1.04360 & 1.68119 \\
\hline & & & & $\mathrm{C}$ & -3.34480 & -3.65649 & 1.97192 \\
\hline \multicolumn{4}{|c|}{ IVa } & $\mathrm{H}$ & -2.64792 & -4.43877 & 1.63211 \\
\hline \multirow{2}{*}{\multicolumn{4}{|c|}{$\begin{array}{l}151 \\
E_{F}=-381304185315\end{array}$}} & $\mathrm{H}$ & -4.34575 & -3.89109 & 1.57953 \\
\hline & & \multicolumn{2}{|c|}{$E_{e}=-3813.04185315$} & $\mathrm{H}$ & -3.40003 & -3.71034 & 3.07036 \\
\hline $\mathrm{C}$ & -3.72240 & 0.08662 & -1.26987 & $\mathrm{C}$ & 2.52185 & -3.33947 & -0.68133 \\
\hline $\mathrm{H}$ & -3.81621 & 1.11225 & -0.89203 & $\mathrm{C}$ & -5.07630 & 0.05646 & 3.55528 \\
\hline $\mathrm{Si}$ & 1.67355 & -0.21432 & 0.48630 & $\mathrm{H}$ & -5.22026 & 0.30989 & 4.60673 \\
\hline $\mathrm{C}$ & -4.12428 & -0.07570 & -2.76603 & $\mathrm{C}$ & 1.25034 & -3.65199 & 0.10092 \\
\hline $\mathrm{Si}$ & 3.51677 & -1.94381 & 2.32851 & $\mathrm{H}$ & 1.17931 & -2.93579 & 0.92852 \\
\hline $\mathrm{C}$ & -5.00370 & -1.92021 & -1.35934 & $\mathrm{C}$ & 4.53110 & -2.01213 & -1.16516 \\
\hline $\mathrm{C}$ & -5.21545 & -1.17358 & -2.69136 & $\mathrm{C}$ & 5.44663 & -0.82181 & -0.89288 \\
\hline $\mathrm{H}$ & -6.21214 & -0.70424 & -2.67714 & $\mathrm{H}$ & 4.78606 & 0.02076 & -0.63136 \\
\hline $\mathrm{H}$ & -5.17945 & -1.84901 & -3.55825 & $\mathrm{C}$ & -0.01230 & -3.47456 & -0.75276 \\
\hline $\mathrm{C}$ & -2.99146 & -0.48140 & -3.71279 & $\mathrm{H}$ & 0.05752 & -4.04530 & -1.69261 \\
\hline $\mathrm{H}$ & -2.20113 & 0.28117 & -3.73636 & $\mathrm{H}$ & -0.18599 & -2.41657 & -0.99219 \\
\hline $\mathrm{H}$ & -3.40088 & -0.58820 & -4.72837 & $\mathrm{H}$ & -0.89602 & -3.83900 & -0.20406 \\
\hline $\mathrm{H}$ & -2.52672 & -1.43271 & -3.42409 & $\mathrm{C}$ & 1.27678 & -5.06085 & 0.70549 \\
\hline $\mathrm{C}$ & -4.70939 & 1.25626 & -3.24981 & $\mathrm{H}$ & 2.17232 & -5.23121 & 1.31859 \\
\hline $\mathrm{H}$ & -3.93199 & 2.03566 & -3.27536 & $\mathrm{H}$ & 1.25137 & -5.83437 & -0.07750 \\
\hline $\mathrm{H}$ & -5.52049 & 1.60111 & -2.59064 & $\mathrm{H}$ & 0.39349 & -5.21074 & 1.34621 \\
\hline $\mathrm{H}$ & -5.11820 & 1.14812 & -4.26556 & $\mathrm{C}$ & 6.38851 & -1.05491 & 0.29348 \\
\hline $\mathrm{C}$ & 2.86171 & -4.18938 & -1.74006 & $\mathrm{H}$ & 5.85114 & -1.19694 & 1.23422 \\
\hline $\mathrm{H}$ & 2.21140 & -5.03639 & -1.97135 & $\mathrm{H}$ & 7.05534 & -0.18829 & 0.42248 \\
\hline $\mathrm{C}$ & -4.01781 & -3.08654 & -1.53749 & $\mathrm{H}$ & 7.01681 & -1.94185 & 0.11378 \\
\hline $\mathrm{H}$ & -3.01136 & -2.72162 & -1.78836 & $\mathrm{~N}$ & 3.05980 & -1.34668 & 0.69550 \\
\hline
\end{tabular}




\begin{tabular}{|c|c|c|c|c|c|c|c|}
\hline $\mathrm{C}$ & 4.54498 & -0.71883 & 3.33922 & $\mathrm{H}$ & -3.12325 & 3.21139 & 0.46635 \\
\hline $\mathrm{H}$ & 3.94083 & -0.30570 & 4.15893 & $\mathrm{H}$ & -2.38220 & 4.29411 & 1.65154 \\
\hline $\mathrm{H}$ & 4.89682 & 0.12841 & 2.73583 & $\mathrm{H}$ & -2.00574 & 4.49202 & -0.07155 \\
\hline $\mathrm{H}$ & 5.42209 & -1.22732 & 3.76981 & $\mathrm{C}$ & 4.84072 & 2.38590 & 0.78061 \\
\hline $\mathrm{C}$ & 4.54232 & -3.50992 & 2.09170 & $\mathrm{H}$ & 4.29020 & 1.46206 & 1.00796 \\
\hline $\mathrm{H}$ & 3.99647 & -4.28581 & 1.53878 & $\mathrm{H}$ & 5.76311 & 2.12238 & 0.23914 \\
\hline $\mathrm{H}$ & 4.78460 & -3.90644 & 3.09095 & $\mathrm{H}$ & 5.12605 & 2.85154 & 1.73708 \\
\hline $\mathrm{H}$ & 5.48532 & -3.33095 & 1.55799 & $\mathrm{C}$ & 4.79622 & 4.58275 & -0.45494 \\
\hline $\mathrm{C}$ & 2.01718 & -2.44253 & 3.36580 & $\mathrm{H}$ & 5.18571 & 5.10737 & 0.43021 \\
\hline $\mathrm{H}$ & 1.58271 & -3.39578 & 3.02947 & $\mathrm{H}$ & 5.66175 & 4.28921 & -1.06750 \\
\hline $\mathrm{H}$ & 1.23360 & -1.67107 & 3.35295 & $\mathrm{H}$ & 4.19334 & 5.29850 & -1.03183 \\
\hline $\mathrm{H}$ & 2.34156 & -2.57291 & 4.41062 & $\mathrm{H}$ & 1.08680 & 1.93374 & -4.09455 \\
\hline $\mathrm{C}$ & 6.31101 & -0.41790 & -2.09273 & & & & \\
\hline $\mathrm{H}$ & 6.77851 & 0.55868 & -1.89651 & $3 \mathbf{a}$ & & & \\
\hline $\mathrm{H}$ & 5.74722 & -0.34273 & -3.03197 & 97 & & & \\
\hline $\mathrm{H}$ & 7.12763 & -1.13948 & -2.24991 & & 5516.72557 & & \\
\hline $\mathrm{N}$ & -4.46029 & -0.82090 & -0.53214 & $\mathrm{C}$ & 0.90427 & 0.33666 & 0.95861 \\
\hline $\mathrm{Cl}$ & 1.96964 & 0.87216 & 2.33034 & $\mathrm{Si}$ & -0.20551 & -0.83585 & -0.04751 \\
\hline $\mathrm{Cl}$ & -1.82707 & -0.12159 & -0.99876 & $\mathrm{C}$ & 0.45586 & 1.02090 & 2.26564 \\
\hline C & 1.79464 & 1.06759 & -0.93686 & $\mathrm{Si}$ & -2.43925 & -0.87966 & -2.22668 \\
\hline $\mathrm{C}$ & 1.77296 & 0.66394 & -2.42656 & $\mathrm{C}$ & 2.86979 & 0.54345 & 2.31092 \\
\hline $\mathrm{C}$ & 0.83814 & 1.71634 & -3.04560 & $\mathrm{C}$ & 1.79273 & 1.44798 & 2.91024 \\
\hline $\mathrm{C}$ & 0.92687 & 2.95438 & -2.16146 & $\mathrm{H}$ & 2.01116 & 2.49354 & 2.64060 \\
\hline $\mathrm{H}$ & -0.19090 & 1.32784 & -3.00208 & $\mathrm{H}$ & 1.76120 & 1.38665 & 4.00728 \\
\hline $\mathrm{N}$ & 1.34466 & 2.32986 & -0.85393 & $\mathrm{C}$ & -0.38554 & 0.13781 & 3.19568 \\
\hline $\mathrm{C}$ & 1.44092 & 3.16397 & 0.32160 & $\mathrm{H}$ & -1.34390 & -0.12178 & 2.72303 \\
\hline $\mathrm{C}$ & 0.29627 & 3.41757 & 1.11057 & $\mathrm{H}$ & -0.60855 & 0.69558 & 4.11830 \\
\hline $\mathrm{C}$ & 2.70067 & 3.69442 & 0.67199 & $\mathrm{H}$ & 0.12706 & -0.79472 & 3.46604 \\
\hline $\mathrm{C}$ & 0.43328 & 4.26324 & 2.21480 & $\mathrm{C}$ & -0.36791 & 2.26568 & 1.92835 \\
\hline $\mathrm{C}$ & 2.78270 & 4.52208 & 1.79495 & $\mathrm{H}$ & -1.36631 & 1.97581 & 1.58855 \\
\hline $\mathrm{C}$ & 1.65925 & 4.81935 & 2.55585 & $\mathrm{H}$ & 0.11126 & 2.87550 & 1.14674 \\
\hline $\mathrm{H}$ & -0.44107 & 4.46235 & 2.83775 & $\mathrm{H}$ & -0.48321 & 2.88602 & 2.83180 \\
\hline $\mathrm{H}$ & 3.75266 & 4.93051 & 2.08478 & $\mathrm{C}$ & -4.42310 & -0.47511 & 2.02989 \\
\hline $\mathrm{H}$ & 1.74425 & 5.46804 & 3.42902 & $\mathrm{H}$ & -4.88218 & -1.21543 & 2.68934 \\
\hline $\mathrm{C}$ & 3.20777 & 0.75141 & -2.97749 & $\mathrm{C}$ & 3.04785 & -0.72195 & 3.15773 \\
\hline $\mathrm{H}$ & 3.75364 & -0.15050 & -2.68281 & $\mathrm{H}$ & 2.13110 & -1.32753 & 3.16634 \\
\hline $\mathrm{H}$ & 3.17793 & 0.78493 & -4.07747 & $\mathrm{H}$ & 3.28146 & -0.43069 & 4.19195 \\
\hline $\mathrm{H}$ & 3.77355 & 1.62170 & -2.61912 & $\mathrm{H}$ & 3.87103 & -1.34412 & 2.79028 \\
\hline $\mathrm{C}$ & 1.24855 & -0.73306 & -2.76248 & $\mathrm{C}$ & -4.85670 & 0.84270 & 2.07704 \\
\hline $\mathrm{H}$ & 1.87580 & -1.52537 & -2.33425 & $\mathrm{H}$ & -5.64508 & 1.13905 & 2.77058 \\
\hline $\mathrm{H}$ & 0.21526 & -0.86369 & -2.41888 & $\mathrm{C}$ & 4.20919 & 1.25204 & 2.14762 \\
\hline $\mathrm{H}$ & 1.26859 & -0.85390 & -3.85767 & $\mathrm{H}$ & 4.95523 & 0.59423 & 1.67969 \\
\hline $\mathrm{C}$ & 3.98743 & 3.34411 & -0.05923 & $\mathrm{H}$ & 4.57925 & 1.54633 & 3.14023 \\
\hline $\mathrm{H}$ & 3.72048 & 2.81221 & -0.97832 & $\mathrm{H}$ & 4.11178 & 2.15632 & 1.53043 \\
\hline $\mathrm{C}$ & -1.05060 & 2.74410 & 0.89058 & $\mathrm{C}$ & -4.28090 & 1.78249 & 1.22720 \\
\hline $\mathrm{H}$ & -0.97247 & 2.12009 & -0.00662 & $\mathrm{H}$ & -4.62595 & 2.81674 & 1.26636 \\
\hline $\mathrm{C}$ & 1.98049 & 3.96615 & -2.62821 & $\mathrm{C}$ & 3.05725 & 0.07098 & -0.19810 \\
\hline $\mathrm{H}$ & 1.62991 & 4.44458 & -3.55346 & $\mathrm{C}$ & 3.21651 & 1.18938 & -1.04345 \\
\hline $\mathrm{H}$ & 2.11904 & 4.75095 & -1.87023 & $\mathrm{C}$ & -2.82056 & 0.08160 & 0.29413 \\
\hline $\mathrm{H}$ & 2.95144 & 3.50137 & -2.83575 & $\mathrm{C}$ & 3.99708 & 1.04227 & -2.19172 \\
\hline $\mathrm{C}$ & -0.41299 & 3.67708 & -2.08402 & $\mathrm{H}$ & 4.12109 & 1.88997 & -2.86754 \\
\hline $\mathrm{H}$ & -0.36942 & 4.51942 & -1.37999 & $\mathrm{C}$ & 3.65576 & -1.16604 & -0.49599 \\
\hline $\mathrm{H}$ & -0.64377 & 4.07900 & -3.08151 & $\mathrm{C}$ & 4.44589 & -1.25607 & -1.64614 \\
\hline $\mathrm{H}$ & -1.22318 & 2.99658 & -1.79348 & $\mathrm{H}$ & 4.91476 & -2.21013 & -1.89554 \\
\hline $\mathrm{C}$ & -1.41627 & 1.80138 & 2.04525 & $\mathrm{C}$ & 3.43816 & -2.41741 & 0.33768 \\
\hline $\mathrm{H}$ & -2.35087 & 1.26743 & 1.80631 & $\mathrm{H}$ & 2.72475 & -2.16715 & 1.13482 \\
\hline $\mathrm{H}$ & -0.62997 & 1.06067 & 2.22872 & $\mathrm{C}$ & 1.25308 & 2.63542 & -1.63802 \\
\hline $\mathrm{H}$ & -1.57573 & 2.36514 & 2.97844 & $\mathrm{H}$ & 0.57783 & 1.78365 & -1.47137 \\
\hline $\mathrm{C}$ & -2.19489 & 3.74942 & 0.71351 & $\mathrm{H}$ & 1.51462 & 2.64730 & -2.70771 \\
\hline
\end{tabular}




\begin{tabular}{|c|c|c|c|}
\hline $\mathrm{H}$ & 0.71348 & 3.56742 & -1.40545 \\
\hline $\mathrm{C}$ & 3.43982 & 3.72041 & -0.99645 \\
\hline $\mathrm{H}$ & 3.70383 & 3.84470 & -2.05689 \\
\hline $\mathrm{H}$ & 4.37530 & 3.62388 & -0.42564 \\
\hline $\mathrm{H}$ & 2.93340 & 4.64323 & -0.67777 \\
\hline $\mathrm{C}$ & 2.51998 & 2.51590 & -0.78094 \\
\hline $\mathrm{H}$ & 2.20889 & 2.52933 & 0.27327 \\
\hline $\mathrm{C}$ & 2.81270 & -3.55485 & -0.47889 \\
\hline $\mathrm{H}$ & 2.62987 & -4.42348 & 0.17104 \\
\hline $\mathrm{H}$ & 3.48510 & -3.87863 & -1.28798 \\
\hline $\mathrm{H}$ & 1.86138 & -3.24581 & -0.92928 \\
\hline $\mathrm{C}$ & 4.75542 & -2.89260 & 0.96454 \\
\hline $\mathrm{H}$ & 4.57418 & -3.70457 & 1.68481 \\
\hline $\mathrm{H}$ & 5.28937 & -2.08122 & 1.48002 \\
\hline $\mathrm{H}$ & 5.43011 & -3.28134 & 0.18643 \\
\hline $\mathrm{C}$ & -3.42509 & -0.88467 & 1.13726 \\
\hline $\mathrm{C}$ & 4.61603 & -0.16703 & -2.49047 \\
\hline $\mathrm{H}$ & 5.22429 & -0.26189 & -3.39123 \\
\hline $\mathrm{C}$ & -3.07526 & -2.36920 & 1.09261 \\
\hline $\mathrm{H}$ & -2.35876 & -2.52584 & 0.27580 \\
\hline $\mathrm{C}$ & -3.26081 & 1.43264 & 0.33823 \\
\hline $\mathrm{C}$ & -2.63048 & 2.51308 & -0.53813 \\
\hline $\mathrm{H}$ & -1.59143 & 2.19818 & -0.72218 \\
\hline $\mathrm{C}$ & -2.40213 & -2.84761 & 2.38356 \\
\hline $\mathrm{H}$ & -3.03393 & -2.63840 & 3.26125 \\
\hline $\mathrm{H}$ & -1.43004 & -2.35870 & 2.52658 \\
\hline $\mathrm{H}$ & -2.22993 & -3.93351 & 2.34117 \\
\hline $\mathrm{C}$ & -4.30748 & -3.23406 & 0.79848 \\
\hline $\mathrm{H}$ & -4.82403 & -2.91723 & -0.11785 \\
\hline $\mathrm{H}$ & -5.03381 & -3.19145 & 1.62431 \\
\hline $\mathrm{H}$ & -4.00862 & -4.28573 & 0.67454 \\
\hline $\mathrm{C}$ & -3.32112 & 2.66342 & -1.89994 \\
\hline $\mathrm{H}$ & -3.23044 & 1.77129 & -2.52583 \\
\hline $\mathrm{H}$ & -2.87360 & 3.50233 & -2.45474 \\
\hline $\mathrm{H}$ & -4.39262 & 2.88024 & -1.76290 \\
\hline $\mathrm{N}$ & -1.80482 & -0.29116 & -0.64827 \\
\hline $\mathrm{C}$ & -1.72381 & 0.06782 & -3.69617 \\
\hline $\mathrm{H}$ & -0.98740 & -0.55040 & -4.22787 \\
\hline $\mathrm{H}$ & -1.20921 & 0.98132 & -3.36513 \\
\hline $\mathrm{H}$ & -2.51986 & 0.35195 & -4.40191 \\
\hline $\mathrm{C}$ & -4.31684 & -0.69286 & -2.19317 \\
\hline $\mathrm{H}$ & -4.70383 & -1.01001 & -3.17510 \\
\hline $\mathrm{H}$ & -4.67400 & 0.32489 & -1.99345 \\
\hline $\mathrm{H}$ & -4.75887 & -1.34769 & -1.42982 \\
\hline $\mathrm{C}$ & -2.15439 & -2.72955 & -2.47884 \\
\hline $\mathrm{H}$ & -2.76393 & -3.32655 & -1.78378 \\
\hline $\mathrm{H}$ & -1.10076 & -3.01922 & -2.36939 \\
\hline $\mathrm{H}$ & -2.46946 & -2.98989 & -3.50235 \\
\hline $\mathrm{C}$ & -2.61685 & 3.89613 & 0.12692 \\
\hline $\mathrm{H}$ & -1.94132 & 4.56609 & -0.42561 \\
\hline $\mathrm{H}$ & -2.29046 & 3.86558 & 1.17463 \\
\hline $\mathrm{H}$ & -3.61625 & 4.35707 & 0.10078 \\
\hline $\mathrm{N}$ & 2.24468 & 0.24105 & 0.97768 \\
\hline $\mathrm{Cl}$ & 0.76543 & -1.01421 & -1.95560 \\
\hline \multicolumn{4}{|c|}{$\begin{array}{l}\text { cAAC.HCl } \\
54 \\
\mathrm{E}_{\mathrm{e}}=-1296.29865413\end{array}$} \\
\hline
\end{tabular}

\begin{tabular}{|c|c|c|c|}
\hline $\mathrm{C}$ & 2.73929 & 0.44633 & -0.15473 \\
\hline $\mathrm{C}$ & 1.32876 & 0.39152 & -0.78946 \\
\hline $\mathrm{C}$ & 0.99168 & 0.33652 & 1.60588 \\
\hline $\mathrm{H}$ & 3.14978 & 0.52239 & 2.01039 \\
\hline $\mathrm{H}$ & 2.38158 & 1.97295 & 1.32688 \\
\hline $\mathrm{N}$ & 0.43130 & 0.13823 & 0.24430 \\
\hline $\mathrm{C}$ & -0.89591 & -0.35717 & 0.01817 \\
\hline $\mathrm{C}$ & -2.01220 & 0.51507 & 0.01635 \\
\hline $\mathrm{C}$ & -1.09203 & -1.74466 & -0.19732 \\
\hline $\mathrm{C}$ & -3.29429 & -0.03297 & -0.11344 \\
\hline $\mathrm{C}$ & -2.39015 & -2.24133 & -0.33787 \\
\hline $\mathrm{C}$ & -3.49178 & -1.39717 & -0.27580 \\
\hline $\mathrm{H}$ & -4.15713 & 0.63604 & -0.10629 \\
\hline $\mathrm{H}$ & -2.53760 & -3.31079 & -0.50224 \\
\hline $\mathrm{H}$ & -4.50113 & -1.79958 & -0.37537 \\
\hline $\mathrm{C}$ & -1.90216 & 2.03151 & 0.05160 \\
\hline $\mathrm{H}$ & -0.84513 & 2.28709 & 0.18128 \\
\hline $\mathrm{C}$ & 0.06528 & -2.71464 & -0.35304 \\
\hline $\mathrm{H}$ & 0.97769 & -2.17158 & -0.09288 \\
\hline $\mathrm{C}$ & 0.19503 & -3.16622 & -1.81319 \\
\hline $\mathrm{H}$ & 1.07986 & -3.80749 & -1.94410 \\
\hline $\mathrm{H}$ & -0.69038 & -3.74250 & -2.12299 \\
\hline $\mathrm{H}$ & 0.28454 & -2.30740 & -2.49425 \\
\hline $\mathrm{C}$ & -0.03967 & -3.92525 & 0.57783 \\
\hline $\mathrm{H}$ & -0.89976 & -4.55972 & 0.31616 \\
\hline $\mathrm{H}$ & 0.86396 & -4.54801 & 0.49657 \\
\hline $\mathrm{H}$ & -0.15357 & -3.61883 & 1.62700 \\
\hline $\mathrm{C}$ & -2.71953 & 2.66816 & 1.18023 \\
\hline $\mathrm{H}$ & -2.54377 & 3.75400 & 1.20495 \\
\hline $\mathrm{H}$ & -3.79853 & 2.51621 & 1.02476 \\
\hline $\mathrm{H}$ & -2.46395 & 2.25905 & 2.16657 \\
\hline $\mathrm{C}$ & -2.34264 & 2.61376 & -1.29825 \\
\hline $\mathrm{H}$ & -3.41447 & 2.43129 & -1.47317 \\
\hline $\mathrm{H}$ & -2.17621 & 3.70154 & -1.31436 \\
\hline $\mathrm{H}$ & -1.77105 & 2.16867 & -2.12214 \\
\hline $\mathrm{C}$ & 3.71954 & 1.41578 & -0.81490 \\
\hline $\mathrm{H}$ & 4.69261 & 1.34467 & -0.30609 \\
\hline $\mathrm{H}$ & 3.86601 & 1.16643 & -1.87611 \\
\hline $\mathrm{H}$ & 3.36566 & 2.45096 & -0.75817 \\
\hline $\mathrm{C}$ & 3.37265 & -0.95320 & -0.21419 \\
\hline $\mathrm{H}$ & 3.51580 & -1.26754 & -1.25918 \\
\hline $\mathrm{H}$ & 4.36057 & -0.92800 & 0.26825 \\
\hline $\mathrm{H}$ & 2.77972 & -1.72136 & 0.29349 \\
\hline $\mathrm{C}$ & 0.20252 & 1.34668 & 2.43952 \\
\hline $\mathrm{H}$ & 0.16120 & 2.33031 & 1.95450 \\
\hline $\mathrm{H}$ & -0.82315 & 0.98817 & 2.60836 \\
\hline $\mathrm{H}$ & 0.68563 & 1.46625 & 3.42047 \\
\hline $\mathrm{C}$ & 1.00989 & -0.98354 & 2.39006 \\
\hline $\mathrm{H}$ & -0.01094 & -1.38814 & 2.46770 \\
\hline $\mathrm{H}$ & 1.64700 & -1.74629 & 1.92615 \\
\hline $\mathrm{H}$ & 1.38219 & -0.80564 & 3.40919 \\
\hline $\mathrm{H}$ & 1.23970 & -0.31143 & -1.62784 \\
\hline $\mathrm{Cl}$ & 0.95666 & 2.01972 & -1.72058 \\
\hline \multicolumn{4}{|c|}{ 1ia } \\
\hline \multicolumn{4}{|c|}{99} \\
\hline \multicolumn{4}{|c|}{$E_{e}=-2975.42425223$} \\
\hline $\mathrm{C}$ & 1.35819 & 0.24109 & 1.19062 \\
\hline $\mathrm{H}$ & -1.04011 & 0.87400 & 1.15891 \\
\hline
\end{tabular}




\begin{tabular}{|c|c|c|c|c|c|c|c|}
\hline & & & & & & & \\
\hline $\mathrm{Si}$ & -0.55517 & -0.07918 & 0.13723 & $\mathrm{H}$ & 4.79060 & 0.17355 & -3.92702 \\
\hline $\mathrm{C}$ & 1.26893 & 0.65636 & 2.66742 & $\mathrm{C}$ & -3.84138 & -1.81020 & 1.37470 \\
\hline $\mathrm{Si}$ & -2.66071 & -1.04532 & -2.10011 & $\mathrm{H}$ & -3.17252 & -2.20881 & 0.59871 \\
\hline $\mathrm{C}$ & 3.50751 & -0.19071 & 2.13181 & $\mathrm{C}$ & -3.47555 & 1.75819 & -0.05151 \\
\hline $\mathrm{C}$ & 2.74057 & 0.65048 & 3.14847 & $\mathrm{C}$ & -2.64739 & 2.57850 & -1.02989 \\
\hline $\mathrm{H}$ & 3.13927 & 1.67725 & 3.15176 & $\mathrm{H}$ & -2.03840 & 1.88053 & -1.61898 \\
\hline $\mathrm{H}$ & 2.84375 & 0.25087 & 4.16667 & $\mathrm{C}$ & -3.14213 & -2.00326 & 2.72409 \\
\hline $\mathrm{C}$ & 0.36453 & -0.23327 & 3.52849 & $\mathrm{H}$ & -3.75941 & -1.59764 & 3.54140 \\
\hline $\mathrm{H}$ & -0.67348 & -0.19275 & 3.17530 & $\mathrm{H}$ & -2.16954 & -1.49677 & 2.74043 \\
\hline $\mathrm{H}$ & 0.38379 & 0.14166 & 4.56269 & $\mathrm{H}$ & -2.96640 & -3.07191 & 2.92119 \\
\hline $\mathrm{H}$ & 0.68259 & -1.28383 & 3.52893 & $\mathrm{C}$ & -5.15241 & -2.60390 & 1.35499 \\
\hline $\mathrm{C}$ & 0.73117 & 2.10143 & 2.72202 & $\mathrm{H}$ & -5.73948 & -2.40160 & 0.44920 \\
\hline $\mathrm{H}$ & -0.34568 & 2.15314 & 2.52130 & $\mathrm{H}$ & -5.78055 & -2.35935 & 2.22532 \\
\hline $\mathrm{H}$ & 1.25215 & 2.75504 & 2.00569 & $\mathrm{H}$ & -4.94301 & -3.68333 & 1.39933 \\
\hline $\mathrm{H}$ & 0.90943 & 2.49801 & 3.73333 & $\mathrm{C}$ & -3.52732 & 3.37454 & -1.99994 \\
\hline $\mathrm{C}$ & -5.04313 & 0.35452 & 1.78879 & $\mathrm{H}$ & -4.29231 & 2.73822 & -2.46689 \\
\hline $\mathrm{H}$ & -5.65729 & -0.19004 & 2.50941 & $\mathrm{H}$ & -2.91323 & 3.81887 & -2.79756 \\
\hline $\mathrm{C}$ & 3.52133 & -1.66613 & 2.54136 & $\mathrm{H}$ & -4.04792 & 4.19732 & -1.48610 \\
\hline $\mathrm{H}$ & 2.50024 & -2.06647 & 2.61614 & $\mathrm{~N}$ & -2.19937 & -0.29251 & -0.56093 \\
\hline $\mathrm{H}$ & 4.00978 & -1.75577 & 3.52203 & $\mathrm{C}$ & -2.65958 & 0.17079 & -3.54239 \\
\hline $\mathrm{H}$ & 4.08343 & -2.27796 & 1.82659 & $\mathrm{H}$ & -1.67858 & 0.65033 & -3.66719 \\
\hline $\mathrm{C}$ & -5.26162 & 1.71412 & 1.59615 & $\mathrm{H}$ & -3.41940 & 0.95461 & -3.41116 \\
\hline $\mathrm{H}$ & -6.04037 & 2.23260 & 2.15785 & $\mathrm{H}$ & -2.89893 & -0.37447 & -4.46936 \\
\hline $\mathrm{C}$ & 4.93126 & 0.28782 & 1.88334 & $\mathrm{C}$ & -4.41446 & -1.72352 & -1.97876 \\
\hline $\mathrm{H}$ & 5.42162 & -0.30739 & 1.10022 & $\mathrm{H}$ & -4.75824 & -1.99010 & -2.99060 \\
\hline $\mathrm{H}$ & 5.50225 & 0.16764 & 2.81514 & $\mathrm{H}$ & -5.11836 & -0.98701 & -1.56255 \\
\hline $\mathrm{H}$ & 4.97031 & 1.34585 & 1.59431 & $\mathrm{H}$ & -4.45636 & -2.63167 & -1.36067 \\
\hline $\mathrm{C}$ & -4.47865 & 2.40424 & 0.67903 & $\mathrm{C}$ & -1.52428 & -2.47894 & -2.53595 \\
\hline $\mathrm{H}$ & -4.64793 & 3.47238 & 0.52384 & $\mathrm{H}$ & -1.30849 & -3.10938 & -1.66092 \\
\hline $\mathrm{C}$ & 3.19472 & -0.03069 & -0.41063 & $\mathrm{H}$ & -0.57353 & -2.12502 & -2.95677 \\
\hline $\mathrm{C}$ & 3.57287 & 1.23056 & -0.91970 & $\mathrm{H}$ & -2.02258 & -3.09993 & -3.29703 \\
\hline $\mathrm{C}$ & -3.24636 & 0.37433 & 0.15372 & $\mathrm{C}$ & -1.68963 & 3.52711 & -0.29842 \\
\hline $\mathrm{C}$ & 4.14142 & 1.27745 & -2.19278 & $\mathrm{H}$ & -1.08108 & 4.09487 & -1.01924 \\
\hline $\mathrm{H}$ & 4.42461 & 2.24143 & -2.61758 & $\mathrm{H}$ & -1.00778 & 2.98027 & 0.36858 \\
\hline $\mathrm{C}$ & 3.40420 & -1.21585 & -1.12983 & $\mathrm{H}$ & -2.25068 & 4.24754 & 0.31796 \\
\hline $\mathrm{C}$ & 3.98845 & -1.11235 & -2.39806 & $\mathrm{~N}$ & 2.60650 & -0.02577 & 0.91496 \\
\hline $\mathrm{H}$ & 4.14811 & -2.02100 & -2.98149 & $\mathrm{Cl}$ & 0.40189 & 0.30936 & -1.73031 \\
\hline $\mathrm{C}$ & 2.99238 & -2.58969 & -0.63510 & $\mathrm{Cl}$ & -0.19045 & -2.09066 & 0.80784 \\
\hline $\mathrm{H}$ & 2.50163 & -2.46768 & 0.33831 & & & & \\
\hline $\mathrm{C}$ & 2.06725 & 3.23324 & -0.64692 & & & & \\
\hline $\mathrm{H}$ & 1.18239 & 2.59956 & -0.50453 & 99 & & & \\
\hline $\mathrm{H}$ & 2.13469 & 3.45918 & -1.72238 & & 2975.4168 & & \\
\hline $\mathrm{H}$ & 1.91647 & 4.17876 & -0.10303 & $\mathrm{C}$ & 1.05342 & -0.82024 & 0.78781 \\
\hline $\mathrm{C}$ & 4.54284 & 3.48111 & -0.25119 & $\mathrm{H}$ & -0.12608 & 0.46542 & 0.80332 \\
\hline $\mathrm{H}$ & 4.66071 & 3.89225 & -1.26423 & $\mathrm{Si}$ & -0.31272 & -0.48425 & -0.51194 \\
\hline $\mathrm{H}$ & 5.48237 & 2.97611 & 0.01775 & $\mathrm{C}$ & 0.69506 & -1.57039 & 2.08122 \\
\hline $\mathrm{H}$ & 4.39872 & 4.33315 & 0.42888 & $\mathrm{Si}$ & -2.61665 & 0.79752 & -2.25368 \\
\hline $\mathrm{C}$ & 3.34237 & 2.53402 & -0.16487 & $\mathrm{C}$ & 2.83160 & -0.36612 & 2.33087 \\
\hline $\mathrm{H}$ & 3.19696 & 2.29346 & 0.89759 & $\mathrm{C}$ & 1.62356 & -0.90141 & 3.10811 \\
\hline $\mathrm{C}$ & 1.95978 & -3.21847 & -1.57334 & $\mathrm{H}$ & 1.08826 & -0.05675 & 3.56946 \\
\hline $\mathrm{H}$ & 1.57985 & -4.15951 & -1.14811 & $\mathrm{H}$ & 1.92482 & -1.58867 & 3.91201 \\
\hline $\mathrm{H}$ & 2.39913 & -3.44223 & -2.55779 & $\mathrm{C}$ & 1.03745 & -3.06094 & 1.88260 \\
\hline $\mathrm{H}$ & 1.10913 & -2.54291 & -1.71702 & $\mathrm{H}$ & 0.35569 & -3.51835 & 1.15517 \\
\hline $\mathrm{C}$ & 4.20747 & -3.51100 & -0.47712 & $\mathrm{H}$ & 0.93019 & -3.57951 & 2.84675 \\
\hline $\mathrm{H}$ & 3.91147 & -4.46057 & -0.00707 & $\mathrm{H}$ & 2.06236 & -3.21926 & 1.52521 \\
\hline $\mathrm{H}$ & 5.00254 & -3.05452 & 0.13095 & $\mathrm{C}$ & -0.76665 & -1.47541 & 2.51262 \\
\hline $\mathrm{H}$ & 4.64542 & -3.74778 & -1.45868 & $\mathrm{H}$ & -1.44129 & -1.87063 & 1.74055 \\
\hline $\mathrm{C}$ & -4.04839 & -0.33159 & 1.08515 & $\mathrm{H}$ & -1.07272 & -0.44244 & 2.72967 \\
\hline $\mathrm{C}$ & 4.34772 & 0.11668 & -2.93170 & $\mathrm{H}$ & -0.91594 & -2.08381 & 3.41704 \\
\hline
\end{tabular}




\begin{tabular}{|c|c|c|c|}
\hline $\mathrm{C}$ & -4.52112 & -0.76554 & 1.85432 \\
\hline $\mathrm{H}$ & -5.22332 & -1.57485 & 2.06709 \\
\hline $\mathrm{C}$ & 4.04258 & -1.30365 & 2.35780 \\
\hline $\mathrm{H}$ & 3.79170 & -2.33424 & 2.08107 \\
\hline $\mathrm{H}$ & 4.46119 & -1.31747 & 3.37387 \\
\hline $\mathrm{H}$ & 4.82225 & -0.93679 & 1.67456 \\
\hline $\mathrm{C}$ & -4.43759 & 0.31101 & 2.72853 \\
\hline $\mathrm{H}$ & -5.06447 & 0.34824 & 3.62079 \\
\hline $\mathrm{C}$ & 3.28010 & 0.98611 & 2.87308 \\
\hline $\mathrm{H}$ & 4.10989 & 1.39246 & 2.27834 \\
\hline $\mathrm{H}$ & 3.63649 & 0.84742 & 3.90415 \\
\hline $\mathrm{H}$ & 2.45756 & 1.71226 & 2.89413 \\
\hline $\mathrm{C}$ & -3.55745 & 1.34730 & 2.44292 \\
\hline $\mathrm{H}$ & -3.50485 & 2.20695 & 3.11494 \\
\hline $\mathrm{C}$ & 3.06846 & 0.28060 & -0.12829 \\
\hline $\mathrm{C}$ & 3.07888 & 1.67541 & -0.35259 \\
\hline $\mathrm{C}$ & -2.80990 & 0.20602 & 0.43039 \\
\hline $\mathrm{C}$ & 3.92487 & 2.17511 & -1.34718 \\
\hline $\mathrm{H}$ & 3.94782 & 3.25078 & -1.53243 \\
\hline $\mathrm{C}$ & 3.82551 & -0.59347 & -0.93724 \\
\hline $\mathrm{C}$ & 4.64386 & -0.04135 & -1.92671 \\
\hline $\mathrm{H}$ & 5.23018 & -0.70626 & -2.56372 \\
\hline $\mathrm{C}$ & 3.72786 & -2.10791 & -0.84570 \\
\hline $\mathrm{H}$ & 3.12699 & -2.35384 & 0.03655 \\
\hline $\mathrm{C}$ & 1.19535 & 3.33198 & -0.63876 \\
\hline $\mathrm{H}$ & 0.57988 & 2.60083 & -1.17366 \\
\hline $\mathrm{H}$ & 1.75189 & 3.91438 & -1.38888 \\
\hline $\mathrm{H}$ & 0.52863 & 4.02838 & -0.10694 \\
\hline $\mathrm{C}$ & 2.92924 & 3.76848 & 1.08922 \\
\hline $\mathrm{H}$ & 3.41844 & 4.43879 & 0.36634 \\
\hline $\mathrm{H}$ & 3.70487 & 3.38043 & 1.75981 \\
\hline $\mathrm{H}$ & 2.23522 & 4.38192 & 1.68392 \\
\hline $\mathrm{C}$ & 2.15766 & 2.66384 & 0.35448 \\
\hline $\mathrm{H}$ & 1.54577 & 2.10300 & 1.07631 \\
\hline $\mathrm{C}$ & 2.99013 & -2.67326 & -2.06588 \\
\hline $\mathrm{H}$ & 2.88255 & -3.76429 & -1.97182 \\
\hline $\mathrm{H}$ & 3.55232 & -2.46385 & -2.98929 \\
\hline $\mathrm{H}$ & 1.98791 & -2.23841 & -2.16839 \\
\hline $\mathrm{C}$ & 5.09610 & -2.77863 & -0.69089 \\
\hline $\mathrm{H}$ & 4.97072 & -3.86045 & -0.53568 \\
\hline $\mathrm{H}$ & 5.66309 & -2.37249 & 0.15839 \\
\hline $\mathrm{H}$ & 5.70740 & -2.64813 & -1.59641 \\
\hline $\mathrm{C}$ & -3.73165 & -0.83963 & 0.70189 \\
\hline $\mathrm{C}$ & 4.71075 & 1.33203 & -2.12268 \\
\hline $\mathrm{H}$ & 5.35898 & 1.74513 & -2.89692 \\
\hline $\mathrm{C}$ & -3.91678 & -2.05469 & -0.20177 \\
\hline $\mathrm{H}$ & -3.26888 & -1.92118 & -1.07827 \\
\hline $\mathrm{C}$ & -2.74060 & 1.31822 & 1.30781 \\
\hline $\mathrm{C}$ & -1.83354 & 2.51355 & 1.05362 \\
\hline $\mathrm{H}$ & -1.19035 & 2.26556 & 0.20058 \\
\hline $\mathrm{C}$ & -3.50167 & -3.35727 & 0.49606 \\
\hline $\mathrm{H}$ & -4.12422 & -3.53641 & 1.38697 \\
\hline $\mathrm{H}$ & -2.44899 & -3.35074 & 0.80144 \\
\hline $\mathrm{H}$ & -3.63809 & -4.20904 & -0.18705 \\
\hline $\mathrm{C}$ & -5.36812 & -2.21207 & -0.67669 \\
\hline $\mathrm{H}$ & -5.75898 & -1.31001 & -1.16304 \\
\hline $\mathrm{H}$ & -6.03611 & -2.44932 & 0.16550 \\
\hline $\mathrm{H}$ & -5.43845 & -3.04225 & -1.39513 \\
\hline $\mathrm{C}$ & -2.64878 & 3.75650 & 0.67925 \\
\hline
\end{tabular}

\begin{tabular}{|lrrr|}
\hline $\mathrm{H}$ & -3.28400 & 3.56991 & -0.19808 \\
$\mathrm{H}$ & -1.98191 & 4.60089 & 0.44388 \\
$\mathrm{~N}$ & -3.30412 & 4.06358 & 1.50921 \\
$\mathrm{C}$ & -1.95825 & 0.14781 & -0.72561 \\
$\mathrm{H}$ & -1.79591 & 2.40398 & -2.81017 \\
$\mathrm{H}$ & -0.79822 & 2.25257 & -3.24055 \\
$\mathrm{H}$ & -2.43531 & 2.86887 & -3.57778 \\
$\mathrm{C}$ & -4.41750 & 1.24312 & -1.94594 \\
$\mathrm{H}$ & -4.83962 & 1.66967 & -2.86910 \\
$\mathrm{H}$ & -4.51853 & 1.98939 & -1.14438 \\
$\mathrm{H}$ & -5.02198 & 0.37444 & -1.66179 \\
$\mathrm{C}$ & -2.49722 & -0.49384 & -3.61254 \\
$\mathrm{H}$ & -3.11473 & -1.37181 & -3.36984 \\
$\mathrm{H}$ & -1.46167 & -0.83464 & -3.75067 \\
$\mathrm{H}$ & -2.85455 & -0.07521 & -4.56610 \\
$\mathrm{C}$ & -0.93069 & 2.81313 & 2.25529 \\
$\mathrm{H}$ & -0.22329 & 3.62276 & 2.01655 \\
$\mathrm{H}$ & -0.35008 & 1.92370 & 2.54597 \\
$\mathrm{H}$ & -1.51590 & 3.13702 & 3.12930 \\
$\mathrm{~N}$ & 2.26493 & -0.27527 & 0.93509 \\
$\mathrm{Cl}$ & 0.70701 & 0.23736 & -2.19228 \\
$\mathrm{Cl}$ & -0.64382 & -2.53132 & -1.08914 \\
& & & \\
\hline
\end{tabular}

\begin{tabular}{|lrll|}
\hline NHC & \multicolumn{4}{|}{} \\
65 & \multicolumn{3}{|}{} \\
$\mathrm{E}_{\mathrm{e}}=-1159.95368177$ & \\
$\mathrm{C}$ & 0.67621 & 0.03721 & 1.92494 \\
$\mathrm{C}$ & -0.00005 & -0.00002 & -0.27063 \\
$\mathrm{C}$ & -0.67621 & -0.03710 & 1.92497 \\
$\mathrm{~N}$ & -1.05724 & -0.05104 & 0.58902 \\
$\mathrm{C}$ & -2.42457 & -0.14850 & 0.17108 \\
$\mathrm{C}$ & -3.23148 & 1.00354 & 0.22810 \\
$\mathrm{C}$ & -2.91334 & -1.39190 & -0.25881 \\
$\mathrm{C}$ & -4.56963 & 0.87890 & -0.15238 \\
$\mathrm{C}$ & -4.25788 & -1.46408 & -0.64095 \\
$\mathrm{C}$ & -5.07887 & -0.34422 & -0.58309 \\
$\mathrm{H}$ & -5.22508 & 1.74940 & -0.11762 \\
$\mathrm{H}$ & -4.66691 & -2.41458 & -0.98750 \\
$\mathrm{H}$ & -6.12631 & -0.42177 & -0.87897 \\
$\mathrm{C}$ & -2.63465 & 2.34937 & 0.60962 \\
$\mathrm{H}$ & -1.87886 & 2.17115 & 1.38986 \\
$\mathrm{C}$ & -2.01251 & -2.60952 & -0.37555 \\
$\mathrm{H}$ & -1.11646 & -2.42557 & 0.23660 \\
$\mathrm{C}$ & -1.55383 & -2.76949 & -1.83014 \\
$\mathrm{H}$ & -0.88253 & -3.63552 & -1.93244 \\
$\mathrm{H}$ & -2.42224 & -2.92800 & -2.48885 \\
$\mathrm{H}$ & -1.02145 & -1.86744 & -2.16348 \\
$\mathrm{C}$ & -2.67360 & -3.88853 & 0.14161 \\
$\mathrm{H}$ & -3.51341 & -4.19995 & -0.49752 \\
$\mathrm{H}$ & -1.94498 & -4.71237 & 0.14309 \\
$\mathrm{H}$ & -3.05297 & -3.76185 & 1.16575 \\
$\mathrm{C}$ & -3.65721 & 3.33286 & 1.17584 \\
$\mathrm{H}$ & -3.14417 & 4.22891 & 1.55301 \\
$\mathrm{H}$ & -4.36602 & 3.66718 & 0.40341 \\
$\mathrm{H}$ & -4.23348 & 2.89224 & 2.00229 \\
$\mathrm{C}$ & -1.91047 & 2.95333 & -0.60158 \\
$\mathrm{H}$ & -2.63206 & 3.16514 & -1.40575 \\
$\mathrm{H}$ & -1.41580 & 3.89751 & -0.32480 \\
\hline & & &
\end{tabular}




\begin{tabular}{|c|c|c|c|c|c|c|c|}
\hline & & & & & & & \\
\hline $\mathrm{H}$ & -1.15305 & 2.26137 & -0.99725 & $\mathrm{H}$ & -0.52750 & -0.86632 & 2.22071 \\
\hline $\mathrm{N}$ & 1.05719 & 0.05092 & 0.58897 & $\mathrm{H}$ & -0.04948 & -2.28972 & 3.17487 \\
\hline $\mathrm{C}$ & 2.42451 & 0.14847 & 0.17101 & $\mathrm{H}$ & -0.72301 & -0.85152 & 3.99758 \\
\hline $\mathrm{C}$ & 3.23143 & -1.00353 & 0.22810 & $\mathrm{C}$ & -2.59620 & -2.86465 & 4.18405 \\
\hline $\mathrm{C}$ & 2.91330 & 1.39189 & -0.25883 & $\mathrm{H}$ & -1.85159 & -3.63880 & 4.42177 \\
\hline $\mathrm{C}$ & 4.56960 & -0.87889 & -0.15231 & $\mathrm{H}$ & -3.57158 & -3.35598 & 4.05646 \\
\hline $\mathrm{C}$ & 4.25787 & 1.46411 & -0.64084 & $\mathrm{H}$ & -2.65712 & -2.19708 & 5.05553 \\
\hline $\mathrm{C}$ & 5.07887 & 0.34426 & -0.58295 & $\mathrm{C}$ & -2.20097 & -2.06488 & 2.94254 \\
\hline $\mathrm{H}$ & 5.22505 & -1.74938 & -0.11754 & $\mathrm{H}$ & -2.90135 & -1.22071 & 2.85844 \\
\hline $\mathrm{H}$ & 4.66690 & 2.41462 & -0.98736 & $\mathrm{C}$ & -2.44975 & -3.00891 & -3.33427 \\
\hline $\mathrm{H}$ & 6.12632 & 0.42181 & -0.87877 & $\mathrm{H}$ & -2.62610 & -2.38615 & -4.22325 \\
\hline $\mathrm{C}$ & 2.63458 & -2.34939 & 0.60952 & $\mathrm{H}$ & -2.97165 & -3.96510 & -3.49573 \\
\hline $\mathrm{H}$ & 1.87857 & -2.17118 & 1.38954 & $\mathrm{H}$ & -1.37173 & -3.20683 & -3.26320 \\
\hline $\mathrm{C}$ & 2.01248 & 2.60950 & -0.37566 & $\mathrm{C}$ & -4.49753 & -2.14056 & -2.18417 \\
\hline $\mathrm{H}$ & 1.11630 & 2.42552 & 0.23628 & $\mathrm{H}$ & -4.77474 & -1.62498 & -3.11609 \\
\hline $\mathrm{C}$ & 1.91072 & -2.95344 & -0.60183 & $\mathrm{H}$ & -4.90755 & -1.56173 & -1.34539 \\
\hline $\mathrm{H}$ & 2.63249 & -3.16523 & -1.40584 & $\mathrm{H}$ & -4.97819 & -3.13124 & -2.18366 \\
\hline $\mathrm{H}$ & 1.41604 & -3.89765 & -0.32516 & $\mathrm{C}$ & 2.78496 & 1.97763 & -0.52947 \\
\hline $\mathrm{H}$ & 1.15335 & -2.26154 & -0.99769 & $\mathrm{C}$ & -1.89561 & -4.94137 & 0.45140 \\
\hline $\mathrm{C}$ & 3.65704 & -3.33278 & 1.17608 & $\mathrm{H}$ & -1.61366 & -5.99548 & 0.45738 \\
\hline $\mathrm{H}$ & 3.14395 & -4.22887 & 1.55310 & $\mathrm{C}$ & 2.04451 & 2.08189 & -1.85336 \\
\hline $\mathrm{H}$ & 4.36614 & -3.66706 & 0.40389 & $\mathrm{H}$ & 2.13089 & 1.11471 & -2.36362 \\
\hline $\mathrm{H}$ & 4.23298 & -2.89207 & 2.00271 & $\mathrm{C}$ & 3.73539 & 0.66918 & 1.31747 \\
\hline $\mathrm{C}$ & 1.55415 & 2.76958 & -1.83035 & $\mathrm{C}$ & 3.98843 & -0.66271 & 2.00327 \\
\hline $\mathrm{H}$ & 0.88285 & 3.63560 & -1.93275 & $\mathrm{H}$ & 3.97277 & -1.44327 & 1.23058 \\
\hline $\mathrm{H}$ & 2.42270 & 2.92812 & -2.48885 & $\mathrm{C}$ & 0.55146 & 2.34676 & -1.61930 \\
\hline $\mathrm{H}$ & 1.02184 & 1.86754 & -2.16386 & $\mathrm{H}$ & 0.40529 & 3.28345 & -1.05745 \\
\hline $\mathrm{C}$ & 2.67348 & 3.88846 & 0.14172 & $\mathrm{H}$ & 0.05903 & 1.54077 & -1.05192 \\
\hline $\mathrm{H}$ & 3.51343 & 4.19993 & -0.49719 & $\mathrm{H}$ & 0.02488 & 2.44131 & -2.58259 \\
\hline $\mathrm{H}$ & 1.94485 & 4.71230 & 0.14310 & $\mathrm{C}$ & 2.63886 & 3.15056 & -2.77487 \\
\hline $\mathrm{H}$ & 3.05262 & 3.76171 & 1.16594 & $\mathrm{H}$ & 3.72729 & 3.03579 & -2.88423 \\
\hline $\mathrm{H}$ & 1.39585 & 0.08013 & 2.73721 & $\mathrm{H}$ & 2.44382 & 4.16487 & -2.39489 \\
\hline $\mathrm{H}$ & -1.39582 & -0.07975 & 2.73728 & $\mathrm{H}$ & 2.18249 & 3.08311 & -3.77331 \\
\hline & & & & $\mathrm{C}$ & 5.33684 & -0.72883 & 2.72144 \\
\hline Ib & & & & $\mathrm{H}$ & 6.16379 & -0.45316 & 2.05142 \\
\hline 112 & & & & $\mathrm{H}$ & 5.51774 & -1.74836 & 3.09265 \\
\hline $\mathrm{E}_{\mathrm{e}}=$ & 3302.11835 & & & $\mathrm{H}$ & 5.36651 & -0.05685 & 3.59237 \\
\hline $\mathrm{C}$ & -2.09773 & 0.12671 & 0.13340 & $\mathrm{~N}$ & 2.83531 & -0.49298 & -0.65800 \\
\hline $\mathrm{Si}$ & 1.22347 & -1.05756 & -0.46840 & $\mathrm{C}$ & 3.63761 & -2.97817 & -2.06111 \\
\hline $\mathrm{Si}$ & 4.06365 & -1.17785 & -1.75504 & $\mathrm{H}$ & 2.63882 & -3.08932 & -2.50937 \\
\hline $\mathrm{C}$ & -4.20201 & -0.40867 & 0.86857 & $\mathrm{H}$ & 3.65983 & -3.56435 & -1.13163 \\
\hline $\mathrm{C}$ & -4.12133 & 0.94437 & 0.83693 & $\mathrm{H}$ & 4.37203 & -3.40503 & -2.76148 \\
\hline $\mathrm{C}$ & 3.07667 & 3.14568 & 0.18500 & $\mathrm{C}$ & 5.75077 & -1.01975 & -0.94612 \\
\hline $\mathrm{H}$ & 2.80367 & 4.11277 & -0.24178 & $\mathrm{H}$ & 6.53472 & -1.26059 & -1.68041 \\
\hline $\mathrm{C}$ & 3.69618 & 3.09476 & 1.42771 & $\mathrm{H}$ & 5.86125 & -1.70634 & -0.09429 \\
\hline $\mathrm{H}$ & 3.91909 & 4.01650 & 1.96783 & $\mathrm{H}$ & 5.92534 & 0.00659 & -0.58807 \\
\hline $\mathrm{C}$ & 4.01654 & 1.86195 & 1.98867 & $\mathrm{C}$ & 4.08507 & -0.25766 & -3.39291 \\
\hline $\mathrm{H}$ & 4.48295 & 1.82508 & 2.97496 & $\mathrm{H}$ & 4.32441 & 0.80720 & -3.25624 \\
\hline $\mathrm{C}$ & -2.60042 & -2.26659 & 0.42956 & $\mathrm{H}$ & 3.10907 & -0.33283 & -3.89582 \\
\hline $\mathrm{C}$ & -2.27457 & -2.87204 & 1.65556 & $\mathrm{H}$ & 4.84844 & -0.69491 & -4.05507 \\
\hline $\mathrm{C}$ & 3.12973 & 0.73249 & 0.04169 & $\mathrm{C}$ & 2.83789 & -0.96329 & 2.97141 \\
\hline $\mathrm{C}$ & -1.93128 & -4.22746 & 1.64315 & $\mathrm{H}$ & 2.94746 & -1.96456 & 3.41509 \\
\hline $\mathrm{H}$ & -1.67081 & -4.72663 & 2.57711 & $\mathrm{H}$ & 1.86421 & -0.92292 & 2.46171 \\
\hline $\mathrm{C}$ & -2.57506 & -2.96779 & -0.79066 & $\mathrm{H}$ & 2.81801 & -0.22072 & 3.78522 \\
\hline $\mathrm{C}$ & -2.20740 & -4.31569 & -0.75199 & $\mathrm{~N}$ & -2.96665 & -0.87669 & 0.43784 \\
\hline $\mathrm{H}$ & -2.16338 & -4.88807 & -1.67826 & $\mathrm{Cl}$ & 1.11987 & -2.97667 & 0.31267 \\
\hline $\mathrm{C}$ & -2.97282 & -2.29005 & -2.09198 & $\mathrm{Cl}$ & 0.32812 & -1.18960 & -2.34380 \\
\hline $\mathrm{H}$ & -2.52551 & -1.28475 & -2.07114 & $\mathrm{H}$ & -5.00738 & -1.07450 & 1.16527 \\
\hline $\mathrm{C}$ & -0.79166 & -1.48018 & 3.09536 & $\mathrm{H}$ & -4.83920 & 1.71295 & 1.10620 \\
\hline
\end{tabular}




\begin{tabular}{|c|c|c|c|c|c|c|c|}
\hline & & & & & & & \\
\hline $\mathrm{N}$ & -2.84545 & 1.23874 & 0.37840 & $\mathrm{H}$ & -0.79028 & -0.76688 & 4.04314 \\
\hline $\mathrm{C}$ & -2.33846 & 2.57220 & 0.24880 & $\mathrm{C}$ & -2.79768 & -2.63484 & 4.24951 \\
\hline $\mathrm{C}$ & -1.52731 & 3.07498 & 1.28274 & $\mathrm{H}$ & -2.09832 & -3.44514 & 4.50239 \\
\hline $\mathrm{C}$ & -2.65372 & 3.33099 & -0.89268 & $\mathrm{H}$ & -3.79937 & -3.07134 & 4.12691 \\
\hline $\mathrm{C}$ & -1.06035 & 4.38858 & 1.17354 & $\mathrm{H}$ & -2.82177 & -1.94941 & 5.10855 \\
\hline $\mathrm{C}$ & -2.16829 & 4.64291 & -0.94761 & $\mathrm{C}$ & -2.35115 & -1.88108 & 2.99715 \\
\hline $\mathrm{C}$ & -1.38740 & 5.17387 & 0.07352 & $\mathrm{H}$ & -2.99933 & -0.99592 & 2.89987 \\
\hline $\mathrm{H}$ & -0.42870 & 4.79828 & 1.96464 & $\mathrm{C}$ & -2.56676 & -2.93105 & -3.26805 \\
\hline $\mathrm{H}$ & -2.40201 & 5.25159 & -1.82432 & $\mathrm{H}$ & -2.63679 & -2.31068 & -4.17301 \\
\hline $\mathrm{H}$ & -1.01908 & 6.19853 & 0.00244 & $\mathrm{H}$ & -3.20008 & -3.81812 & -3.42532 \\
\hline $\mathrm{C}$ & -1.14163 & 2.24339 & 2.49480 & $\mathrm{H}$ & -1.52242 & -3.24922 & -3.15741 \\
\hline $\mathrm{H}$ & -1.57339 & 1.24089 & 2.37290 & $\mathrm{C}$ & -4.53147 & -1.81483 & -2.19488 \\
\hline $\mathrm{C}$ & -3.49998 & 2.84837 & -2.06714 & $\mathrm{H}$ & -4.72997 & -1.31519 & -3.15491 \\
\hline $\mathrm{H}$ & -3.18288 & 3.48698 & -2.90719 & $\mathrm{H}$ & -4.90245 & -1.15973 & -1.39495 \\
\hline $\mathrm{C}$ & -1.71428 & 2.84276 & 3.78271 & $\mathrm{H}$ & -5.11460 & -2.74821 & -2.16775 \\
\hline $\mathrm{H}$ & -1.46051 & 2.20884 & 4.64552 & $\mathrm{C}$ & 2.89396 & 1.79484 & -0.50646 \\
\hline $\mathrm{H}$ & -1.30366 & 3.84671 & 3.97141 & $\mathrm{C}$ & -2.31513 & -4.81845 & 0.55861 \\
\hline $\mathrm{H}$ & -2.80926 & 2.92976 & 3.72903 & $\mathrm{H}$ & -2.15514 & -5.89723 & 0.58681 \\
\hline $\mathrm{C}$ & 0.37915 & 2.07936 & 2.58414 & $\mathrm{C}$ & 2.19692 & 1.98245 & -1.84399 \\
\hline $\mathrm{H}$ & 0.64523 & 1.39482 & 3.40416 & $\mathrm{H}$ & 2.17644 & 1.00952 & -2.34970 \\
\hline $\mathrm{H}$ & 0.79740 & 1.67582 & 1.64845 & $\mathrm{C}$ & 3.66025 & 0.37719 & 1.34015 \\
\hline $\mathrm{H}$ & 0.87678 & 3.04261 & 2.77712 & $\mathrm{C}$ & 3.77403 & -0.98304 & 2.00718 \\
\hline $\mathrm{C}$ & -4.98919 & 3.13464 & -1.83612 & $\mathrm{H}$ & 3.68454 & -1.74342 & 1.21949 \\
\hline $\mathrm{H}$ & -5.38621 & 2.50567 & -1.02610 & $\mathrm{C}$ & 0.74224 & 2.40713 & -1.62880 \\
\hline $\mathrm{H}$ & -5.15894 & 4.18782 & -1.57076 & $\mathrm{H}$ & 0.68757 & 3.34081 & -1.04640 \\
\hline $\mathrm{H}$ & -5.56576 & 2.90848 & -2.74548 & $\mathrm{H}$ & 0.18037 & 1.63901 & -1.07500 \\
\hline $\mathrm{C}$ & -3.28720 & 1.39883 & -2.51924 & $\mathrm{H}$ & 0.23183 & 2.56823 & -2.59228 \\
\hline $\mathrm{H}$ & -2.22506 & 1.11376 & -2.50403 & $\mathrm{C}$ & 2.91860 & 2.97656 & -2.75650 \\
\hline $\mathrm{H}$ & -3.83766 & 0.68668 & -1.88702 & $\mathrm{H}$ & 3.98936 & 2.74113 & -2.84388 \\
\hline $\mathrm{H}$ & -3.66684 & 1.28380 & -3.54588 & $\mathrm{H}$ & 2.83037 & 4.00892 & -2.38440 \\
\hline $\mathrm{H}$ & 0.48286 & -0.16001 & 0.41402 & $\mathrm{H}$ & 2.47760 & 2.95302 & -3.76399 \\
\hline & & & & $\mathrm{C}$ & 5.10160 & -1.19259 & 2.73623 \\
\hline & & & & $\mathrm{H}$ & 5.95862 & -0.96801 & 2.08498 \\
\hline 11 & & & & $\mathrm{H}$ & 5.18669 & -2.23644 & 3.07276 \\
\hline & 3302.08582 & & & $\mathrm{H}$ & 5.17912 & -0.55653 & 3.63125 \\
\hline $\mathrm{C}$ & -1.96684 & 0.28051 & 0.25316 & $\mathrm{~N}$ & 2.59170 & -0.65315 & -0.62437 \\
\hline $\mathrm{Si}$ & 0.88100 & -1.03540 & -0.48628 & $\mathrm{C}$ & 3.31818 & -3.25963 & -1.94592 \\
\hline $\mathrm{Si}$ & 3.76482 & -1.45487 & -1.70526 & $\mathrm{H}$ & 2.31817 & -3.37217 & -2.38776 \\
\hline $\mathrm{C}$ & -4.12723 & -0.08095 & 0.78252 & $\mathrm{H}$ & 3.33041 & -3.80910 & -0.99453 \\
\hline $\mathrm{C}$ & -3.93452 & 1.26175 & 0.75290 & $\mathrm{H}$ & 4.05509 & -3.71267 & -2.62785 \\
\hline $\mathrm{C}$ & 3.35533 & 2.91524 & 0.19492 & $\mathrm{C}$ & 5.48184 & -1.33235 & -0.93953 \\
\hline $\mathrm{H}$ & 3.23462 & 3.90760 & -0.24467 & $\mathrm{H}$ & 6.22551 & -1.66681 & -1.67977 \\
\hline $\mathrm{C}$ & 3.96656 & 2.78566 & 1.43664 & $\mathrm{H}$ & 5.58287 & -1.97006 & -0.04948 \\
\hline $\mathrm{H}$ & 4.32839 & 3.66871 & 1.96636 & $\mathrm{H}$ & 5.72872 & -0.29820 & -0.65449 \\
\hline $\mathrm{C}$ & 4.11349 & 1.52154 & 2.00154 & $\mathrm{C}$ & 3.84554 & -0.61968 & -3.39237 \\
\hline $\mathrm{H}$ & 4.59029 & 1.42270 & 2.97865 & $\mathrm{H}$ & 4.19182 & 0.42177 & -3.31796 \\
\hline $\mathrm{C}$ & -2.68870 & -2.08923 & 0.47705 & $\mathrm{H}$ & 2.86024 & -0.63053 & -3.88080 \\
\hline $\mathrm{C}$ & -2.47233 & -2.70084 & 1.72311 & $\mathrm{H}$ & 4.55644 & -1.16660 & -4.03177 \\
\hline $\mathrm{C}$ & 3.04505 & 0.51463 & 0.07268 & $\mathrm{C}$ & 2.59019 & -1.19391 & 2.95458 \\
\hline $\mathrm{C}$ & -2.28911 & -4.08521 & 1.73892 & $\mathrm{H}$ & 2.60493 & -2.20734 & 3.38433 \\
\hline $\mathrm{H}$ & -2.10851 & -4.59491 & 2.68532 & $\mathrm{H}$ & 1.63910 & -1.06877 & 2.41895 \\
\hline $\mathrm{C}$ & -2.73604 & -2.80458 & -0.73255 & $\mathrm{H}$ & 2.61869 & -0.46243 & 3.77895 \\
\hline $\mathrm{C}$ & -2.53416 & -4.18566 & -0.65982 & $\mathrm{~N}$ & -2.90988 & -0.66011 & 0.46733 \\
\hline $\mathrm{H}$ & -2.54107 & -4.77684 & -1.57459 & $\mathrm{Cl}$ & 0.79168 & -3.04909 & 0.24083 \\
\hline $\mathrm{C}$ & -3.03365 & -2.12230 & -2.05874 & $\mathrm{Cl}$ & 0.31041 & -1.29186 & -2.56712 \\
\hline $\mathrm{H}$ & -2.47523 & -1.17408 & -2.06570 & $\mathrm{H}$ & -5.00438 & -0.67919 & 1.00980 \\
\hline $\mathrm{C}$ & -0.90694 & -1.39148 & 3.14316 & $\mathrm{H}$ & -4.60600 & 2.09116 & 0.95040 \\
\hline $\mathrm{H}$ & -0.59105 & -0.80330 & 2.26814 & $\mathrm{~N}$ & -2.60700 & 1.45734 & 0.41378 \\
\hline $\mathrm{H}$ & -0.22287 & -2.25013 & 3.21707 & $\mathrm{C}$ & -2.02634 & 2.76543 & 0.26691 \\
\hline
\end{tabular}




\begin{tabular}{|c|c|c|c|c|c|c|c|}
\hline & & & & & & & \\
\hline C & -1.26292 & 3.27110 & 1.33384 & $\mathrm{H}$ & 2.95863 & 3.41299 & 4.37876 \\
\hline C & -2.26696 & 3.49197 & -0.91170 & $\mathrm{H}$ & 4.55338 & 3.11359 & 3.64391 \\
\hline C & -0.74912 & 4.56481 & 1.20741 & $\mathrm{H}$ & 3.83568 & 1.92637 & 4.76363 \\
\hline C & -1.74699 & 4.79021 & -0.97325 & C & 2.93652 & 1.90888 & 2.79449 \\
\hline $\mathrm{C}$ & -1.00223 & 5.32589 & 0.07089 & $\mathrm{H}$ & 3.58403 & 1.05912 & 2.52476 \\
\hline $\mathrm{H}$ & -0.14201 & 4.98073 & 2.01253 & $\mathrm{C}$ & 1.49426 & 3.10242 & -3.25111 \\
\hline $\mathrm{H}$ & -1.91797 & 5.37993 & -1.87674 & $\mathrm{H}$ & 1.32533 & 2.49620 & -4.15136 \\
\hline $\mathrm{H}$ & -0.60037 & 6.33697 & -0.00940 & $\mathrm{H}$ & 2.00404 & 4.03047 & -3.55476 \\
\hline C & -0.93424 & 2.43508 & 2.55867 & $\mathrm{H}$ & 0.51109 & 3.34354 & -2.82432 \\
\hline $\mathrm{H}$ & -1.65681 & 1.60560 & 2.61320 & $\mathrm{C}$ & 3.74035 & 2.06118 & -2.82915 \\
\hline C & -3.05008 & 2.99877 & -2.12605 & $\mathrm{H}$ & 3.66947 & 1.56113 & -3.80617 \\
\hline $\mathrm{H}$ & -2.62624 & 3.57417 & -2.96437 & $\mathrm{H}$ & 4.36313 & 1.43339 & -2.17494 \\
\hline C & -1.04843 & 3.22539 & 3.86375 & $\mathrm{H}$ & 4.26242 & 3.01974 & -2.97171 \\
\hline $\mathrm{H}$ & -0.91808 & 2.54858 & 4.72040 & C & -2.92654 & -1.89763 & 0.15498 \\
\hline $\mathrm{H}$ & -0.26776 & 3.99649 & 3.93985 & $\mathrm{C}$ & 2.21794 & 4.91061 & 0.54300 \\
\hline $\mathrm{H}$ & -2.02736 & 3.71723 & 3.95726 & $\mathrm{H}$ & 2.01382 & 5.97685 & 0.64918 \\
\hline C & 0.46622 & 1.83126 & 2.40006 & $\mathrm{C}$ & -2.49965 & -2.33128 & -1.23826 \\
\hline $\mathrm{H}$ & 0.72458 & 1.20924 & 3.26975 & $\mathrm{H}$ & -2.59686 & -1.46326 & -1.90114 \\
\hline $\mathrm{H}$ & 0.53857 & 1.20300 & 1.49812 & C & -3.32840 & -0.16139 & 1.83615 \\
\hline $\mathrm{H}$ & 1.22563 & 2.62331 & 2.30482 & C & -3.33812 & 1.29960 & 2.25481 \\
\hline C & -4.53061 & 3.39017 & -2.03053 & $\mathrm{H}$ & -3.45461 & 1.90025 & 1.34242 \\
\hline $\mathrm{H}$ & -5.03632 & 2.82545 & -1.23352 & C & -1.01991 & -2.72728 & -1.24516 \\
\hline $\mathrm{H}$ & -4.64992 & 4.46258 & -1.82136 & $\mathrm{H}$ & -0.83361 & -3.56963 & -0.55886 \\
\hline $\mathrm{H}$ & -5.04511 & 3.16173 & -2.97547 & $\mathrm{H}$ & -0.38999 & -1.88349 & -0.92253 \\
\hline C & -2.90121 & 1.51998 & -2.50105 & $\mathrm{H}$ & -0.69807 & -3.02353 & -2.25694 \\
\hline $\mathrm{H}$ & -1.86152 & 1.16791 & -2.42564 & $\mathrm{C}$ & -3.35929 & -3.46609 & -1.79958 \\
\hline $\mathrm{H}$ & -3.53683 & 0.87082 & -1.88066 & $\mathrm{H}$ & -4.43213 & -3.23982 & -1.71594 \\
\hline $\mathrm{H}$ & -3.22870 & 1.38151 & -3.54225 & $\mathrm{H}$ & -3.17480 & -4.41538 & -1.27268 \\
\hline $\mathrm{H}$ & -0.59780 & -0.12679 & 0.04079 & $\mathrm{H}$ & -3.12452 & -3.62937 & -2.86196 \\
\hline & & & & C & -4.47879 & 1.64510 & 3.21290 \\
\hline IIb & & & & $\mathrm{H}$ & -5.44878 & 1.30886 & 2.81862 \\
\hline 112 & & & & $\mathrm{H}$ & -4.52656 & 2.73341 & 3.36626 \\
\hline & 3302.09959 & & & $\mathrm{H}$ & -4.33549 & 1.18397 & 4.20243 \\
\hline $\mathrm{C}$ & 2.15507 & -0.18651 & 0.09943 & $\mathrm{~N}$ & -2.76332 & 0.48705 & -0.46809 \\
\hline $\mathrm{Si}$ & -1.03865 & 0.88413 & -0.66004 & C & -3.84845 & 2.75234 & -2.15260 \\
\hline $\mathrm{Si}$ & -4.14839 & 1.04969 & -1.42815 & $\mathrm{H}$ & -2.95287 & 2.75613 & -2.78974 \\
\hline $\mathrm{C}$ & 4.31213 & 0.28913 & 0.19240 & $\mathrm{H}$ & -3.70799 & 3.51043 & -1.37064 \\
\hline $\mathrm{C}$ & 4.18407 & -1.06564 & 0.16259 & $\mathrm{H}$ & -4.72178 & 3.02472 & -2.76663 \\
\hline $\mathrm{C}$ & -3.18729 & -2.86780 & 1.12979 & $\mathrm{C}$ & -5.66856 & 1.10807 & -0.31064 \\
\hline $\mathrm{H}$ & -3.12614 & -3.92502 & 0.86113 & $\mathrm{H}$ & -6.56373 & 1.30485 & -0.92127 \\
\hline $\mathrm{C}$ & -3.51915 & -2.51000 & 2.43187 & $\mathrm{H}$ & -5.59018 & 1.90899 & 0.43955 \\
\hline $\mathrm{H}$ & -3.72654 & -3.27884 & 3.17860 & $\mathrm{H}$ & -5.82088 & 0.15466 & 0.21855 \\
\hline $\mathrm{C}$ & -3.57887 & -1.16271 & 2.77862 & $\mathrm{C}$ & -4.54530 & -0.12192 & -2.85243 \\
\hline $\mathrm{H}$ & -3.82827 & -0.88368 & 3.80447 & $\mathrm{H}$ & -4.82353 & -1.12566 & -2.49949 \\
\hline $\mathrm{C}$ & 2.71728 & 2.22454 & 0.26997 & $\mathrm{H}$ & -3.67906 & -0.21021 & -3.52518 \\
\hline $\mathrm{C}$ & 2.74810 & 2.77784 & 1.56146 & $\mathrm{H}$ & -5.39338 & 0.28132 & -3.42830 \\
\hline $\mathrm{C}$ & -3.01091 & -0.52902 & 0.50555 & $\mathrm{C}$ & -1.98205 & 1.68086 & 2.85852 \\
\hline $\mathrm{C}$ & 2.49577 & 4.14609 & 1.67205 & $\mathrm{H}$ & -1.96524 & 2.74541 & 3.13940 \\
\hline $\mathrm{H}$ & 2.50447 & 4.61882 & 2.65419 & $\mathrm{H}$ & -1.17128 & 1.51601 & 2.13378 \\
\hline $\mathrm{C}$ & 2.41295 & 2.95793 & -0.88673 & $\mathrm{H}$ & -1.77395 & 1.07651 & 3.75761 \\
\hline $\mathrm{C}$ & 2.16902 & 4.32345 & -0.71503 & $\mathrm{~N}$ & 3.03546 & 0.81426 & 0.15208 \\
\hline $\mathrm{H}$ & 1.90446 & 4.93001 & -1.57970 & $\mathrm{Cl}$ & -1.02522 & 3.07498 & -0.48924 \\
\hline $\mathrm{C}$ & 2.33705 & 2.30551 & -2.25755 & $\mathrm{Cl}$ & -0.88689 & 0.72474 & -2.88394 \\
\hline $\mathrm{H}$ & 1.82928 & 1.33407 & -2.13247 & $\mathrm{H}$ & 5.18606 & 0.93045 & 0.25229 \\
\hline $\mathrm{C}$ & 1.57670 & 1.34323 & 3.22399 & $\mathrm{H}$ & 4.92159 & -1.86063 & 0.19560 \\
\hline $\mathrm{H}$ & 1.07035 & 0.81893 & 2.39910 & $\mathrm{~N}$ & 2.83167 & -1.33600 & 0.09343 \\
\hline $\mathrm{H}$ & 0.90947 & 2.15937 & 3.53958 & $\mathrm{C}$ & 2.26084 & -2.66121 & 0.05767 \\
\hline $\mathrm{H}$ & 1.69162 & 0.64195 & 4.06539 & $\mathrm{C}$ & 1.72284 & -3.17300 & 1.25158 \\
\hline $\mathrm{C}$ & 3.61287 & 2.63716 & 3.95539 & $\mathrm{C}$ & 2.29356 & -3.38004 & -1.14817 \\
\hline
\end{tabular}




\begin{tabular}{|c|c|c|c|c|c|c|c|}
\hline & \multirow[b]{2}{*}{1.27141} & & & & & & \\
\hline $\mathrm{C}$ & & -4.49554 & 1.23247 & $\mathrm{H}$ & -3.58695 & 3.54505 & 1.97214 \\
\hline $\mathrm{C}$ & 1.83855 & -4.70234 & -1.10121 & $\mathrm{C}$ & -3.37046 & 1.37827 & 1.96295 \\
\hline $\mathrm{C}$ & 1.35105 & -5.26159 & 0.07429 & $\mathrm{H}$ & -2.27948 & 1.44415 & 1.85103 \\
\hline $\mathrm{H}$ & 0.84393 & -4.92749 & 2.13796 & $\mathrm{C}$ & -3.18096 & -2.91734 & -2.57291 \\
\hline $\mathrm{H}$ & 1.85009 & -5.29214 & -2.02032 & $\mathrm{H}$ & -2.66399 & -3.06869 & -3.53122 \\
\hline $\mathrm{H}$ & 0.99886 & -6.29401 & 0.07961 & $\mathrm{H}$ & -4.13711 & -3.46067 & -2.62051 \\
\hline $\mathrm{C}$ & 1.53789 & -2.31865 & 2.49445 & $\mathrm{H}$ & -2.56125 & -3.35378 & -1.78062 \\
\hline $\mathrm{H}$ & 2.21855 & -1.45416 & 2.42816 & $\mathrm{C}$ & -4.38428 & -0.89716 & -3.40866 \\
\hline $\mathrm{C}$ & 2.76592 & -2.84132 & -2.49531 & $\mathrm{H}$ & -3.99512 & -1.10056 & -4.41696 \\
\hline $\mathrm{H}$ & 2.22318 & -3.45907 & -3.22768 & $\mathrm{H}$ & -4.57626 & 0.18104 & -3.32885 \\
\hline $\mathrm{C}$ & 1.88488 & -3.06275 & 3.78548 & $\mathrm{H}$ & -5.35364 & -1.40979 & -3.31001 \\
\hline $\mathrm{H}$ & 1.85133 & -2.36588 & 4.63514 & $\mathrm{C}$ & 3.35263 & -0.06134 & 0.66170 \\
\hline $\mathrm{H}$ & 1.16085 & -3.86291 & 3.99724 & $\mathrm{C}$ & -5.38044 & -1.69261 & 0.89953 \\
\hline $\mathrm{H}$ & 2.88894 & -3.50866 & 3.74246 & $\mathrm{H}$ & -6.15739 & -2.33588 & 1.31526 \\
\hline $\mathrm{C}$ & 0.09430 & -1.79401 & 2.53408 & $\mathrm{C}$ & 4.18971 & -0.52729 & -0.52752 \\
\hline $\mathrm{H}$ & -0.06998 & -1.17076 & 3.42570 & $\mathrm{H}$ & 4.37188 & -1.59895 & -0.35309 \\
\hline $\mathrm{H}$ & -0.16467 & -1.19215 & 1.64617 & $\mathrm{C}$ & 1.65925 & -0.53710 & 2.36802 \\
\hline $\mathrm{H}$ & -0.61904 & -2.63173 & 2.56855 & $\mathrm{C}$ & 0.63906 & -1.45738 & 3.02483 \\
\hline $\mathrm{C}$ & 4.25986 & -3.10583 & -2.72169 & $\mathrm{H}$ & 0.47096 & -2.30612 & 2.34970 \\
\hline $\mathrm{H}$ & 4.87883 & -2.48516 & -2.05695 & $\mathrm{C}$ & 3.50667 & -0.39209 & -1.88800 \\
\hline $\mathrm{H}$ & 4.51348 & -4.16035 & -2.54348 & $\mathrm{H}$ & 3.24166 & 0.65890 & -2.05604 \\
\hline $\mathrm{H}$ & 4.53505 & -2.85367 & -3.75607 & $\mathrm{H}$ & 2.60263 & -1.00631 & -1.96937 \\
\hline $\mathrm{C}$ & 2.40666 & -1.38532 & -2.81485 & $\mathrm{H}$ & 4.19429 & -0.70518 & -2.68936 \\
\hline $\mathrm{H}$ & 1.37496 & -1.12509 & -2.52915 & $\mathrm{C}$ & 5.56497 & 0.13864 & -0.59084 \\
\hline $\mathrm{H}$ & 3.10295 & -0.67347 & -2.34530 & $\mathrm{H}$ & 6.10968 & 0.05862 & 0.36053 \\
\hline $\mathrm{H}$ & 2.48775 & -1.22651 & -3.90006 & $\mathrm{H}$ & 5.47882 & 1.20630 & -0.84916 \\
\hline \multirow[t]{2}{*}{$\mathrm{H}$} & 1.04535 & -0.03740 & 0.02128 & $\mathrm{H}$ & 6.17033 & -0.34036 & -1.37338 \\
\hline & & & & $\mathrm{C}$ & 1.17471 & -2.00474 & 4.35429 \\
\hline \multicolumn{4}{|c|}{$[\mathbf{I I b}-2 \mathrm{~b}]^{*}$} & $\mathrm{H}$ & 2.16523 & -2.46455 & 4.23456 \\
\hline \multicolumn{4}{|c|}{112} & $\mathrm{H}$ & 0.48697 & -2.76292 & 4.75753 \\
\hline \multicolumn{4}{|c|}{$E_{e}=-3302.07373706$} & $\mathrm{H}$ & 1.26848 & -1.20670 & 5.10674 \\
\hline $\mathrm{C}$ & -1.04266 & 0.90772 & -0.35644 & $\mathrm{~N}$ & 1.65444 & -1.81995 & 0.25286 \\
\hline $\mathrm{Si}$ & 0.14125 & -1.34208 & -0.54677 & $\mathrm{C}$ & 1.06181 & -4.77504 & 0.40984 \\
\hline $\mathrm{Si}$ & 2.37836 & -3.45388 & 0.19375 & $\mathrm{H}$ & 0.33951 & -4.75301 & -0.41866 \\
\hline $\mathrm{C}$ & -2.64956 & 1.62307 & -1.78522 & $\mathrm{H}$ & 0.50099 & -4.65078 & 1.34702 \\
\hline $\mathrm{C}$ & -1.54799 & 2.35598 & -2.02918 & $\mathrm{H}$ & 1.55153 & -5.76177 & 0.41887 \\
\hline $\mathrm{C}$ & 3.76550 & 1.03544 & 1.42105 & $\mathrm{C}$ & 3.59140 & -3.55572 & 1.62975 \\
\hline $\mathrm{H}$ & 4.61082 & 1.63519 & 1.08549 & $\mathrm{H}$ & 4.10879 & -4.52713 & 1.60679 \\
\hline $\mathrm{C}$ & 3.13751 & 1.37371 & 2.61795 & $\mathrm{H}$ & 3.05647 & -3.47852 & 2.58737 \\
\hline $\mathrm{H}$ & 3.47476 & 2.24153 & 3.18803 & $\mathrm{H}$ & 4.35065 & -2.76011 & 1.60567 \\
\hline $\mathrm{C}$ & 2.12317 & 0.56378 & 3.10173 & $\mathrm{C}$ & 3.27795 & -3.85673 & -1.41516 \\
\hline $\mathrm{H}$ & 1.67785 & 0.77782 & 4.07682 & $\mathrm{H}$ & 4.15378 & -3.22372 & -1.61193 \\
\hline $\mathrm{C}$ & -3.38895 & -0.07206 & -0.14585 & $\mathrm{H}$ & 2.59517 & -3.77866 & -2.27196 \\
\hline $\mathrm{C}$ & -3.85849 & 0.18060 & 1.15982 & $\mathrm{H}$ & 3.62594 & -4.90057 & -1.35171 \\
\hline $\mathrm{C}$ & 2.21990 & -0.80329 & 1.09631 & $\mathrm{C}$ & -0.71245 & -0.77788 & 3.25979 \\
\hline $\mathrm{C}$ & -4.84547 & -0.66474 & 1.67073 & $\mathrm{H}$ & -1.43009 & -1.49301 & 3.69072 \\
\hline $\mathrm{H}$ & -5.21757 & -0.51118 & 2.68295 & $\mathrm{H}$ & -1.15776 & -0.41691 & 2.32185 \\
\hline $\mathrm{C}$ & -3.90978 & -1.11040 & -0.94169 & $\mathrm{H}$ & -0.61523 & 0.07114 & 3.95684 \\
\hline $\mathrm{C}$ & -4.92565 & -1.90053 & -0.39505 & $\mathrm{~N}$ & -2.38155 & 0.78348 & -0.70566 \\
\hline $\mathrm{H}$ & -5.34425 & -2.71443 & -0.98861 & $\mathrm{Cl}$ & -1.37867 & -2.51102 & 0.46361 \\
\hline $\mathrm{C}$ & -3.40633 & -1.41803 & -2.34532 & $\mathrm{Cl}$ & 0.19990 & -2.44949 & -2.39445 \\
\hline $\mathrm{H}$ & -2.43292 & -0.91907 & -2.47909 & $\mathrm{H}$ & -3.62578 & 1.63258 & -2.25523 \\
\hline $\mathrm{C}$ & -3.64756 & 1.26389 & 3.46073 & $\mathrm{H}$ & -1.36332 & 3.13816 & -2.75619 \\
\hline $\mathrm{H}$ & -3.27481 & 0.31494 & 3.87101 & $\mathrm{~N}$ & -0.56910 & 1.95874 & -1.12598 \\
\hline $\mathrm{H}$ & -4.72388 & 1.33717 & 3.67781 & $\mathrm{C}$ & 0.64215 & 2.67017 & -0.87292 \\
\hline $\mathrm{H}$ & -3.15160 & 2.08674 & 3.99554 & $\mathrm{C}$ & 0.74005 & 3.37838 & 0.34556 \\
\hline $\mathrm{C}$ & -3.97142 & 2.67831 & 1.41200 & $\mathrm{C}$ & 1.66884 & 2.71433 & -1.83803 \\
\hline $\mathrm{H}$ & -5.06710 & 2.66275 & 1.51498 & $\mathrm{C}$ & 1.94687 & 4.00791 & 0.65315 \\
\hline $\mathrm{H}$ & -3.73008 & 2.82534 & 0.34948 & $\mathrm{C}$ & 2.84933 & 3.38210 & -1.48354 \\
\hline
\end{tabular}




\begin{tabular}{|c|c|c|c|c|c|c|c|}
\hline & & & & & & & \\
\hline $\mathrm{C}$ & 3.00629 & 3.99404 & -0.24680 & $\mathrm{H}$ & -1.97335 & 1.08824 & 2.21566 \\
\hline $\mathrm{H}$ & 2.04650 & 4.53983 & 1.60179 & $\mathrm{C}$ & -3.63216 & -1.47920 & -3.27650 \\
\hline $\mathrm{H}$ & 3.66659 & 3.41060 & -2.20885 & $\mathrm{H}$ & -3.23425 & -1.36153 & -4.29539 \\
\hline $\mathrm{H}$ & 3.94793 & 4.48465 & 0.00497 & $\mathrm{H}$ & -4.65132 & -1.88588 & -3.36391 \\
\hline $\mathrm{C}$ & -0.46196 & 3.59744 & 1.25375 & $\mathrm{H}$ & -3.01026 & -2.21376 & -2.74933 \\
\hline $\mathrm{H}$ & -1.33342 & 3.10762 & 0.79690 & $\mathrm{C}$ & -4.55426 & 0.85106 & -3.30279 \\
\hline $\mathrm{C}$ & 1.59706 & 2.19521 & -3.27351 & $\mathrm{H}$ & -4.23480 & 0.96428 & -4.34930 \\
\hline $\mathrm{H}$ & 2.64013 & 1.94857 & -3.52281 & $\mathrm{H}$ & -4.56614 & 1.84697 & -2.83863 \\
\hline $\mathrm{C}$ & -0.79538 & 5.09259 & 1.32926 & $\mathrm{H}$ & -5.58900 & 0.47440 & -3.30233 \\
\hline $\mathrm{H}$ & -1.71294 & 5.24936 & 1.91567 & $\mathrm{C}$ & 3.24780 & -0.76636 & 0.41018 \\
\hline $\mathrm{H}$ & 0.01370 & 5.65783 & 1.81551 & $\mathrm{C}$ & -5.60962 & -1.04179 & 0.55607 \\
\hline $\mathrm{H}$ & -0.94953 & 5.51556 & 0.32635 & $\mathrm{H}$ & -6.51251 & -1.60497 & 0.79741 \\
\hline $\mathrm{C}$ & -0.26562 & 3.00876 & 2.64996 & $\mathrm{C}$ & 3.86125 & -1.09211 & -0.94356 \\
\hline $\mathrm{H}$ & -1.15656 & 3.19091 & 3.27065 & $\mathrm{H}$ & 3.28849 & -1.92791 & -1.36332 \\
\hline $\mathrm{H}$ & -0.08368 & 1.92580 & 2.60981 & $\mathrm{C}$ & 1.59366 & -1.24024 & 2.15808 \\
\hline $\mathrm{H}$ & 0.60028 & 3.46494 & 3.15516 & $\mathrm{C}$ & 0.46559 & -2.11145 & 2.69439 \\
\hline $\mathrm{C}$ & 1.20262 & 3.32732 & -4.23406 & $\mathrm{H}$ & 0.08452 & -2.71012 & 1.85993 \\
\hline $\mathrm{H}$ & 0.17558 & 3.67637 & -4.05333 & $\mathrm{C}$ & 3.73278 & 0.05637 & -1.93364 \\
\hline $\mathrm{H}$ & 1.87142 & 4.19312 & -4.12872 & $\mathrm{H}$ & 4.30902 & 0.92944 & -1.59384 \\
\hline $\mathrm{H}$ & 1.25497 & 2.97225 & -5.27379 & $\mathrm{H}$ & 2.68334 & 0.35998 & -2.01847 \\
\hline $\mathrm{C}$ & 0.77959 & 0.92774 & -3.53436 & $\mathrm{H}$ & 4.09485 & -0.24141 & -2.92987 \\
\hline $\mathrm{H}$ & 0.82338 & 0.23083 & -2.68998 & $\mathrm{C}$ & 5.32263 & -1.53116 & -0.81315 \\
\hline $\mathrm{H}$ & -0.27944 & 1.13722 & -3.73703 & $\mathrm{H}$ & 5.44811 & -2.31861 & -0.05714 \\
\hline $\mathrm{H}$ & 1.18290 & 0.40438 & -4.41324 & $\mathrm{H}$ & 5.96877 & -0.68586 & -0.53239 \\
\hline \multirow[t]{2}{*}{$\mathrm{H}$} & -0.74115 & 0.85467 & 0.69023 & $\mathrm{H}$ & 5.68849 & -1.91458 & -1.77765 \\
\hline & & & & $\mathrm{C}$ & 1.01774 & -3.07992 & 3.74740 \\
\hline \multicolumn{4}{|c|}{$2 \mathbf{b}$} & $\mathrm{H}$ & 1.88968 & -3.63118 & 3.36450 \\
\hline \multirow{2}{*}{\multicolumn{4}{|c|}{$\begin{array}{l}112 \\
E_{e}=-3302.08711895\end{array}$}} & $\mathrm{H}$ & 0.24692 & -3.80837 & 4.04136 \\
\hline & & & & $\mathrm{H}$ & 1.33601 & -2.54255 & 4.65383 \\
\hline $\mathrm{C}$ & -0.64319 & 0.69738 & -0.15513 & $\mathrm{~N}$ & 1.17254 & -1.98209 & -0.16017 \\
\hline $\mathrm{Si}$ & -0.20216 & -1.12270 & -0.77860 & $\mathrm{C}$ & 0.38715 & -4.87961 & 0.66778 \\
\hline $\mathrm{Si}$ & 1.41891 & -3.75300 & -0.43618 & $\mathrm{H}$ & -0.68561 & -4.65200 & 0.63844 \\
\hline $\mathrm{C}$ & -2.13572 & 2.22548 & -1.14477 & $\mathrm{H}$ & 0.72698 & -4.86086 & 1.71272 \\
\hline $\mathrm{C}$ & -0.91299 & 2.66994 & -1.42268 & $\mathrm{H}$ & 0.53024 & -5.90635 & 0.29312 \\
\hline $\mathrm{C}$ & 3.95127 & 0.02107 & 1.32472 & $\mathrm{C}$ & 3.20791 & -4.18805 & -0.04946 \\
\hline $\mathrm{H}$ & 4.89055 & 0.48588 & 1.01791 & $\mathrm{H}$ & 3.25462 & -5.26896 & 0.15376 \\
\hline $\mathrm{C}$ & 3.48264 & 0.21362 & 2.62164 & $\mathrm{H}$ & 3.56785 & -3.66264 & 0.84888 \\
\hline $\mathrm{H}$ & 4.03671 & 0.84905 & 3.31450 & $\mathrm{H}$ & 3.89724 & -3.96954 & -0.87587 \\
\hline $\mathrm{C}$ & 2.33646 & -0.44957 & 3.04266 & $\mathrm{C}$ & 1.00455 & -4.14605 & -2.22185 \\
\hline $\mathrm{H}$ & 2.01389 & -0.36702 & 4.08384 & $\mathrm{H}$ & 1.57061 & -3.51670 & -2.92308 \\
\hline $\mathrm{C}$ & -3.26684 & 0.35195 & -0.05500 & $\mathrm{H}$ & -0.06639 & -4.00789 & -2.43076 \\
\hline $\mathrm{C}$ & -3.72509 & 0.30439 & 1.28055 & $\mathrm{H}$ & 1.25574 & -5.20074 & -2.41409 \\
\hline $\mathrm{C}$ & 2.00500 & -1.31192 & 0.80878 & $\mathrm{C}$ & -0.73240 & -1.34283 & 3.24297 \\
\hline $\mathrm{C}$ & -4.87837 & -0.42926 & 1.56929 & $\mathrm{H}$ & -1.48073 & -2.04894 & 3.63358 \\
\hline $\mathrm{H}$ & -5.23071 & -0.50060 & 2.59829 & $\mathrm{H}$ & -1.22258 & -0.75911 & 2.45180 \\
\hline $\mathrm{C}$ & -4.02829 & -0.22527 & -1.09642 & $\mathrm{H}$ & -0.45372 & -0.66023 & 4.06157 \\
\hline $\mathrm{C}$ & -5.20095 & -0.91001 & -0.76477 & $\mathrm{~N}$ & -2.06878 & 1.06081 & -0.36848 \\
\hline $\mathrm{H}$ & -5.79264 & -1.36833 & -1.55967 & $\mathrm{Cl}$ & -1.81648 & -2.37430 & -0.31678 \\
\hline $\mathrm{C}$ & -3.62949 & -0.12405 & -2.56120 & $\mathrm{Cl}$ & -0.17405 & -1.06423 & -2.85629 \\
\hline $\mathrm{H}$ & -2.60568 & 0.27014 & -2.61147 & $\mathrm{H}$ & -3.10256 & 2.63626 & -1.41959 \\
\hline $\mathrm{C}$ & -3.37521 & 0.70254 & 3.78052 & $\mathrm{H}$ & -0.60428 & 3.56071 & -1.95898 \\
\hline $\mathrm{H}$ & -3.20624 & -0.37361 & 3.92246 & $\mathrm{~N}$ & 0.05983 & 1.80153 & -0.86213 \\
\hline $\mathrm{H}$ & -4.42094 & 0.92165 & 4.04499 & $\mathrm{C}$ & 1.22845 & 2.43871 & -0.32660 \\
\hline $\mathrm{H}$ & -2.73874 & 1.24653 & 4.49392 & $\mathrm{C}$ & 1.26870 & 2.83813 & 1.03347 \\
\hline $\mathrm{C}$ & -3.46253 & 2.61940 & 2.16854 & $\mathrm{C}$ & 2.29321 & 2.78884 & -1.18711 \\
\hline $\mathrm{H}$ & -4.55508 & 2.72524 & 2.25008 & $\mathrm{C}$ & 2.46755 & 3.30766 & 1.57092 \\
\hline $\mathrm{H}$ & -3.15005 & 3.00621 & 1.18782 & $\mathrm{C}$ & 3.46807 & 3.28488 & -0.59940 \\
\hline $\mathrm{H}$ & -3.00017 & 3.24519 & 2.94778 & $\mathrm{C}$ & 3.58634 & 3.49043 & 0.76661 \\
\hline $\mathrm{C}$ & -3.06075 & 1.14960 & 2.35390 & $\mathrm{H}$ & 2.50865 & 3.58502 & 2.62660 \\
\hline
\end{tabular}




\begin{tabular}{|c|c|c|c|c|c|c|c|}
\hline & & & & & & & \\
\hline $\mathrm{H}$ & 4.31203 & 3.52088 & -1.25369 & $\mathrm{H}$ & -2.69449 & -0.33980 & -4.31268 \\
\hline $\mathrm{H}$ & 4.52467 & 3.84990 & 1.19219 & $\mathrm{H}$ & -3.77652 & -1.71559 & -4.01229 \\
\hline C & 0.00344 & 3.00853 & 1.86579 & $\mathrm{H}$ & -2.13282 & -1.69433 & -3.30910 \\
\hline $\mathrm{H}$ & -0.84879 & 2.59201 & 1.31462 & $\mathrm{C}$ & -4.76684 & 0.49151 & -2.62694 \\
\hline C & 2.28778 & 2.86544 & -2.72086 & $\mathrm{H}$ & -4.55525 & 1.20853 & -3.43418 \\
\hline $\mathrm{H}$ & 3.31002 & 2.57565 & -3.01604 & $\mathrm{H}$ & -5.09858 & 1.05825 & -1.74338 \\
\hline C & -0.28197 & 4.51046 & 2.01057 & $\mathrm{H}$ & -5.60131 & -0.15181 & -2.94737 \\
\hline $\mathrm{H}$ & -1.22395 & 4.67307 & 2.55484 & $\mathrm{C}$ & 3.89984 & 0.25729 & -0.36730 \\
\hline $\mathrm{H}$ & 0.52503 & 5.01466 & 2.56335 & $\mathrm{C}$ & -4.74954 & -3.36401 & -0.31599 \\
\hline $\mathrm{H}$ & -0.36733 & 4.98691 & 1.02328 & $\mathrm{H}$ & -5.28178 & -4.29834 & -0.50050 \\
\hline C & 0.08025 & 2.33104 & 3.23267 & $\mathrm{C}$ & 3.65289 & 0.98636 & -1.67750 \\
\hline $\mathrm{H}$ & -0.85053 & 2.49455 & 3.79699 & $\mathrm{H}$ & 2.97788 & 0.36191 & -2.27776 \\
\hline $\mathrm{H}$ & 0.24111 & 1.24902 & 3.13105 & $\mathrm{C}$ & 3.31291 & -1.44773 & 1.28850 \\
\hline $\mathrm{H}$ & 0.90840 & 2.73626 & 3.83416 & $\mathrm{C}$ & 2.41978 & -2.56520 & 1.80281 \\
\hline $\mathrm{C}$ & 2.11809 & 4.34220 & -3.12140 & $\mathrm{H}$ & 1.81620 & -2.91798 & 0.95924 \\
\hline $\mathrm{H}$ & 1.12540 & 4.71540 & -2.82540 & $\mathrm{C}$ & 2.93277 & 2.31205 & -1.41797 \\
\hline $\mathrm{H}$ & 2.86849 & 4.98477 & -2.64123 & $\mathrm{H}$ & 3.57261 & 2.98778 & -0.82788 \\
\hline $\mathrm{H}$ & 2.20833 & 4.45435 & -4.21205 & $\mathrm{H}$ & 2.00055 & 2.16130 & -0.85384 \\
\hline $\mathrm{C}$ & 1.34172 & 2.01138 & -3.56530 & $\mathrm{H}$ & 2.67720 & 2.81389 & -2.36443 \\
\hline $\mathrm{H}$ & 1.35594 & 0.95307 & -3.29724 & $\mathrm{C}$ & 4.93535 & 1.22150 & -2.47928 \\
\hline $\mathrm{H}$ & 0.29876 & 2.34696 & -3.51031 & $\mathrm{H}$ & 5.50202 & 0.28985 & -2.61700 \\
\hline $\mathrm{H}$ & 1.65665 & 2.09798 & -4.61645 & $\mathrm{H}$ & 5.59537 & 1.95037 & -1.98426 \\
\hline \multirow[t]{2}{*}{$\mathrm{H}$} & -0.36957 & 0.69293 & 0.92126 & $\mathrm{H}$ & 4.69099 & 1.62704 & -3.47261 \\
\hline & & & & $\mathrm{C}$ & 3.21691 & -3.75057 & 2.35467 \\
\hline \multicolumn{4}{|c|}{ 1ib } & $\mathrm{H}$ & 3.99872 & -4.07516 & 1.65327 \\
\hline 112 & & & & $\mathrm{H}$ & 2.54823 & -4.60350 & 2.54440 \\
\hline \multicolumn{4}{|c|}{$E_{e}=-3299.59504605$} & $\mathrm{H}$ & 3.70744 & -3.49988 & 3.30791 \\
\hline $\mathrm{C}$ & -1.50094 & 0.66490 & 0.19357 & $\mathrm{~N}$ & 1.97329 & -1.22393 & -0.77754 \\
\hline $\mathrm{Si}$ & 0.40232 & -0.42389 & -0.43817 & $\mathrm{C}$ & 2.32703 & -4.23300 & -1.13121 \\
\hline $\mathrm{Si}$ & 2.29921 & -2.52651 & -1.93671 & $\mathrm{H}$ & 1.36493 & -4.46049 & -0.64840 \\
\hline $\mathrm{C}$ & -3.53165 & 1.28013 & 1.00789 & $\mathrm{H}$ & 3.12548 & -4.32334 & -0.38111 \\
\hline $\mathrm{C}$ & -2.70366 & 2.34063 & 1.15299 & $\mathrm{H}$ & 2.50937 & -4.99081 & -1.90957 \\
\hline $\mathrm{C}$ & 4.94201 & 0.67449 & 0.46588 & $\mathrm{C}$ & 3.99397 & -2.26125 & -2.71723 \\
\hline $\mathrm{H}$ & 5.57838 & 1.50421 & 0.15054 & $\mathrm{H}$ & 4.27354 & -3.15533 & -3.29594 \\
\hline $\mathrm{C}$ & 5.18533 & 0.05074 & 1.68591 & $\mathrm{H}$ & 4.76833 & -2.09610 & -1.95219 \\
\hline $\mathrm{H}$ & 6.00559 & 0.38724 & 2.32226 & $\mathrm{H}$ & 3.99691 & -1.40066 & -3.40209 \\
\hline $\mathrm{C}$ & 4.37530 & -1.00567 & 2.08540 & $\mathrm{C}$ & 1.00950 & -2.59175 & -3.30396 \\
\hline $\mathrm{H}$ & 4.56239 & -1.49337 & 3.04472 & $\mathrm{H}$ & 1.01974 & -1.68964 & -3.92966 \\
\hline $\mathrm{C}$ & -3.40849 & -0.99711 & 0.15087 & $\mathrm{H}$ & -0.00358 & -2.72315 & -2.89758 \\
\hline $\mathrm{C}$ & -3.63713 & -1.86252 & 1.23140 & $\mathrm{H}$ & 1.23742 & -3.46316 & -3.93825 \\
\hline $\mathrm{C}$ & 3.06648 & -0.81141 & 0.04719 & $\mathrm{C}$ & 1.44374 & -2.05129 & 2.86623 \\
\hline $\mathrm{C}$ & -4.32623 & -3.04951 & 0.97155 & $\mathrm{H}$ & 0.78648 & -2.86449 & 3.20927 \\
\hline $\mathrm{H}$ & -4.51910 & -3.74673 & 1.78893 & $\mathrm{H}$ & 0.80752 & -1.24138 & 2.47505 \\
\hline $\mathrm{C}$ & -3.80247 & -1.29483 & -1.16135 & $\mathrm{H}$ & 1.99166 & -1.66149 & 3.73879 \\
\hline $\mathrm{C}$ & -4.48069 & -2.49847 & -1.37132 & $\mathrm{~N}$ & -2.78297 & 0.27424 & 0.42244 \\
\hline $\mathrm{H}$ & -4.79985 & -2.76222 & -2.38076 & $\mathrm{Cl}$ & -0.68297 & -2.23225 & -0.14434 \\
\hline $\mathrm{C}$ & -3.52435 & -0.34936 & -2.31469 & $\mathrm{Cl}$ & 0.07239 & 0.42766 & -2.35647 \\
\hline $\mathrm{H}$ & -2.72550 & 0.33254 & -2.00049 & $\mathrm{H}$ & -4.57919 & 1.13956 & 1.25411 \\
\hline $\mathrm{C}$ & -2.31648 & -2.75379 & 3.18341 & $\mathrm{H}$ & -2.86574 & 3.33396 & 1.55868 \\
\hline $\mathrm{H}$ & -1.53081 & -3.05359 & 2.47665 & $\mathrm{~N}$ & -1.48281 & 1.95567 & 0.63438 \\
\hline $\mathrm{H}$ & -2.96372 & -3.62273 & 3.37522 & $\mathrm{C}$ & -0.38504 & 2.89012 & 0.56864 \\
\hline $\mathrm{H}$ & -1.84259 & -2.47705 & 4.13688 & $\mathrm{C}$ & 0.58190 & 2.84501 & 1.59550 \\
\hline $\mathrm{C}$ & -4.25892 & -1.19396 & 3.58083 & $\mathrm{C}$ & -0.36975 & 3.87135 & -0.43933 \\
\hline $\mathrm{H}$ & -4.97764 & -2.02327 & 3.66857 & $\mathrm{C}$ & 1.62160 & 3.77442 & 1.55723 \\
\hline $\mathrm{H}$ & -4.80919 & -0.31041 & 3.22736 & $\mathrm{C}$ & 0.70276 & 4.77623 & -0.42542 \\
\hline $\mathrm{H}$ & -3.86879 & -0.97481 & 4.58563 & $\mathrm{C}$ & 1.68687 & 4.73135 & 0.54954 \\
\hline $\mathrm{C}$ & -3.11781 & -1.57159 & 2.63021 & $\mathrm{H}$ & 2.39225 & 3.75090 & 2.32714 \\
\hline $\mathrm{H}$ & -2.42874 & -0.71527 & 2.56450 & $\mathrm{H}$ & 0.74733 & 5.54012 & -1.20509 \\
\hline $\mathrm{C}$ & -3.00642 & -1.07483 & -3.55625 & $\mathrm{H}$ & 2.51122 & 5.44571 & 0.52666 \\
\hline
\end{tabular}




\begin{tabular}{|c|c|c|c|c|c|c|c|}
\hline & & & & & & & \\
\hline $\mathrm{C}$ & 0.46301 & 1.88827 & 2.77143 & $\mathrm{H}$ & $\begin{array}{l}-3.19810 \\
\end{array}$ & -0.83323 & -3.34708 \\
\hline $\mathrm{H}$ & -0.09937 & 1.00311 & 2.43760 & $\mathrm{C}$ & -4.94850 & 1.68330 & -1.68308 \\
\hline $\mathrm{C}$ & -1.45760 & 4.17051 & -1.47367 & $\mathrm{H}$ & -4.77257 & 2.47719 & -2.42390 \\
\hline $\mathrm{H}$ & -0.90455 & 4.59250 & -2.32769 & $\mathrm{H}$ & -4.90166 & 2.13230 & -0.67932 \\
\hline $\mathrm{C}$ & -0.33789 & 2.54945 & 3.90148 & $\mathrm{H}$ & -5.96775 & 1.29344 & -1.83162 \\
\hline $\mathrm{H}$ & -0.45069 & 1.85582 & 4.74776 & $\mathrm{C}$ & 3.59447 & -0.12398 & -0.67343 \\
\hline $\mathrm{H}$ & 0.18121 & 3.45058 & 4.26218 & $\mathrm{C}$ & -5.31864 & -2.49089 & -0.01967 \\
\hline $\mathrm{H}$ & -1.34236 & 2.84750 & 3.56810 & $\mathrm{H}$ & -6.07356 & -3.26095 & -0.18624 \\
\hline $\mathrm{C}$ & 1.82203 & 1.40820 & 3.27873 & $\mathrm{C}$ & 3.37288 & 0.55305 & -2.01835 \\
\hline $\mathrm{H}$ & 1.68118 & 0.63621 & 4.04758 & $\mathrm{H}$ & 2.55875 & 0.01434 & -2.52327 \\
\hline $\mathrm{H}$ & 2.42906 & 0.97871 & 2.46643 & $\mathrm{C}$ & 2.87423 & -1.62416 & 1.12934 \\
\hline $\mathrm{H}$ & 2.39158 & 2.22634 & 3.74422 & $\mathrm{C}$ & 1.88406 & -2.58277 & 1.77530 \\
\hline $\mathrm{C}$ & -2.36861 & 5.28947 & -0.94483 & $\mathrm{H}$ & 0.94951 & -2.52982 & 1.20001 \\
\hline $\mathrm{H}$ & -2.97259 & 4.93481 & -0.09629 & $\mathrm{C}$ & 2.91434 & 2.00125 & -1.84025 \\
\hline $\mathrm{H}$ & -1.78860 & 6.15992 & -0.60810 & $\mathrm{H}$ & 3.70808 & 2.59496 & -1.36075 \\
\hline $\mathrm{H}$ & -3.06300 & 5.61719 & -1.73233 & $\mathrm{H}$ & 2.01705 & 2.06826 & -1.21248 \\
\hline $\mathrm{C}$ & -2.29751 & 3.02635 & -2.03960 & $\mathrm{H}$ & 2.67662 & 2.45490 & -2.81451 \\
\hline $\mathrm{H}$ & -1.66942 & 2.19587 & -2.37789 & $\mathrm{C}$ & 4.60375 & 0.51835 & -2.92968 \\
\hline $\mathrm{H}$ & -3.03639 & 2.64456 & -1.32079 & $\mathrm{H}$ & 5.03968 & -0.48743 & -3.01211 \\
\hline $\mathrm{H}$ & -2.86210 & 3.40527 & -2.90472 & $\mathrm{H}$ & 5.39223 & 1.19688 & -2.57047 \\
\hline \multirow{2}{*}{$\mathrm{H}$} & 0.93737 & 0.41097 & 0.65547 & $\mathrm{H}$ & 4.32529 & 0.85521 & -3.93883 \\
\hline & & & & $\mathrm{C}$ & 2.41112 & -4.02250 & 1.72033 \\
\hline \multicolumn{4}{|c|}{$[1 \mathrm{ib}-2 \mathrm{~b}]^{\dagger}$} & $\mathrm{H}$ & 2.70735 & -4.30886 & 0.70180 \\
\hline \multicolumn{4}{|c|}{112} & $\mathrm{H}$ & 1.63922 & -4.72876 & 2.06278 \\
\hline \multicolumn{4}{|c|}{$E_{e}=-3299.53342843$} & $\mathrm{H}$ & 3.29056 & -4.14238 & 2.37209 \\
\hline $\mathrm{C}$ & -1.10397 & 0.66518 & 0.35113 & $\mathrm{~N}$ & 1.39537 & -1.23901 & -0.80765 \\
\hline $\mathrm{Si}$ & -0.15605 & -0.45170 & -0.81754 & $\mathrm{C}$ & 0.78343 & -4.25112 & -1.27395 \\
\hline $\mathrm{Si}$ & 1.64239 & -2.68390 & -1.89551 & $\mathrm{H}$ & -0.08798 & -4.47815 & -1.90396 \\
\hline $\mathrm{C}$ & -2.81779 & 1.48784 & 1.62716 & $\mathrm{H}$ & 0.42695 & -4.16057 & -0.23902 \\
\hline $\mathrm{C}$ & -1.77846 & 2.31861 & 1.78214 & $\mathrm{H}$ & 1.47955 & -5.10251 & -1.32471 \\
\hline $\mathrm{C}$ & 4.84367 & -0.02163 & -0.05276 & $\mathrm{C}$ & 3.48946 & -3.02942 & -1.96397 \\
\hline $\mathrm{H}$ & 5.62070 & 0.58694 & -0.51799 & $\mathrm{H}$ & 3.62944 & -3.94085 & -2.56698 \\
\hline $\mathrm{C}$ & 5.13399 & -0.72625 & 1.11098 & $\mathrm{H}$ & 3.94245 & -3.19243 & -0.97546 \\
\hline $\mathrm{H}$ & 6.12529 & -0.65931 & 1.56214 & $\mathrm{H}$ & 4.03501 & -2.21197 & -2.45357 \\
\hline $\mathrm{C}$ & 4.15067 & -1.51626 & 1.69205 & $\mathrm{C}$ & 1.08261 & -2.34542 & -3.65700 \\
\hline $\mathrm{H}$ & 4.37864 & -2.07472 & 2.60322 & $\mathrm{H}$ & 1.60616 & -1.48203 & -4.09238 \\
\hline $\mathrm{C}$ & -3.40116 & -0.53095 & 0.40536 & $\mathrm{H}$ & 0.00253 & -2.16450 & -3.73347 \\
\hline $\mathrm{C}$ & -3.58030 & -1.52954 & 1.37535 & $\mathrm{H}$ & 1.32818 & -3.23686 & -4.25679 \\
\hline $\mathrm{C}$ & 2.59462 & -0.93012 & -0.07191 & $\mathrm{C}$ & 1.56125 & -2.20236 & 3.22330 \\
\hline $\mathrm{C}$ & -4.55628 & -2.50473 & 1.14140 & $\mathrm{H}$ & 0.92722 & -2.97405 & 3.68289 \\
\hline $\mathrm{H}$ & -4.71254 & -3.29276 & 1.88114 & $\mathrm{H}$ & 1.02399 & -1.24411 & 3.28012 \\
\hline $\mathrm{C}$ & -4.15148 & -0.50690 & -0.78456 & $\mathrm{H}$ & 2.47674 & -2.12629 & 3.83096 \\
\hline $\mathrm{C}$ & -5.11191 & -1.50006 & -0.97616 & $\mathrm{~N}$ & -2.46941 & 0.52847 & 0.67361 \\
\hline $\mathrm{H}$ & -5.70280 & -1.50324 & -1.89342 & $\mathrm{Cl}$ & -1.45454 & -2.01622 & -1.26466 \\
\hline $\mathrm{C}$ & -3.91945 & 0.55826 & -1.83739 & $\mathrm{Cl}$ & -0.12987 & 0.61661 & -2.67275 \\
\hline $\mathrm{H}$ & -2.92430 & 0.98328 & -1.65759 & $\mathrm{H}$ & -3.80266 & 1.47653 & 2.08329 \\
\hline $\mathrm{C}$ & -1.94058 & -2.91930 & 2.66241 & $\mathrm{H}$ & -1.64801 & 3.19630 & 2.40588 \\
\hline $\mathrm{H}$ & -1.29224 & -2.99491 & 1.77604 & $\mathrm{~N}$ & -0.75374 & 1.88861 & 0.93017 \\
\hline $\mathrm{H}$ & -2.60980 & -3.79305 & 2.67323 & $\mathrm{C}$ & 0.38218 & 2.74273 & 0.72175 \\
\hline $\mathrm{H}$ & -1.31240 & -2.96432 & 3.56323 & $\mathrm{C}$ & 1.53906 & 2.51609 & 1.50570 \\
\hline $\mathrm{C}$ & -3.58862 & -1.44847 & 3.89539 & $\mathrm{C}$ & 0.26911 & 3.87338 & -0.11533 \\
\hline $\mathrm{H}$ & -4.33010 & -2.25777 & 3.97893 & $\mathrm{C}$ & 2.61862 & 3.39051 & 1.35794 \\
\hline $\mathrm{H}$ & -4.13410 & -0.49348 & 3.88997 & $\mathrm{C}$ & 1.38968 & 4.70952 & -0.22792 \\
\hline $\mathrm{H}$ & -2.95633 & -1.47692 & 4.79497 & $\mathrm{C}$ & 2.55108 & 4.47379 & 0.48861 \\
\hline $\mathrm{C}$ & -2.73412 & -1.60991 & 2.63489 & $\mathrm{H}$ & 3.52827 & 3.22976 & 1.93282 \\
\hline $\mathrm{H}$ & -2.00237 & -0.78959 & 2.61393 & $\mathrm{H}$ & 1.32713 & 5.57615 & -0.89002 \\
\hline $\mathrm{C}$ & -3.90940 & 0.00036 & -3.26067 & $\mathrm{H}$ & 3.40789 & 5.14060 & 0.37866 \\
\hline $\mathrm{H}$ & -3.59950 & 0.78703 & -3.96404 & $\mathrm{C}$ & 1.59846 & 1.39502 & 2.53731 \\
\hline $\mathrm{H}$ & -4.90444 & -0.35089 & -3.57402 & $\mathrm{H}$ & 1.26739 & 0.47127 & 2.03336 \\
\hline
\end{tabular}




\begin{tabular}{|lrrl|}
\hline $\mathrm{C}$ & -0.98038 & 4.38526 & -0.83041 \\
$\mathrm{H}$ & -0.58833 & 4.91577 & -1.71271 \\
$\mathrm{C}$ & 0.67329 & 1.63810 & 3.74079 \\
$\mathrm{H}$ & 0.92034 & 0.92143 & 4.53849 \\
$\mathrm{H}$ & 0.82513 & 2.65139 & 4.14533 \\
$\mathrm{H}$ & -0.38880 & 1.50795 & 3.50490 \\
$\mathrm{C}$ & 3.01176 & 1.17169 & 3.06739 \\
$\mathrm{H}$ & 3.04163 & 0.25000 & 3.66395 \\
$\mathrm{H}$ & 3.74406 & 1.07345 & 2.25845 \\
$\mathrm{H}$ & 3.31590 & 2.00334 & 3.72325 \\
$\mathrm{C}$ & -1.67888 & 5.44001 & 0.04180 \\
$\mathrm{H}$ & -2.12414 & 4.97437 & 0.93313 \\
$\mathrm{H}$ & -0.97925 & 6.21973 & 0.37435 \\
$\mathrm{H}$ & -2.49095 & 5.91986 & -0.52424 \\
$\mathrm{C}$ & -1.99189 & 3.37223 & -1.35171 \\
$\mathrm{H}$ & -1.50286 & 2.57527 & -1.92266 \\
$\mathrm{H}$ & -2.58433 & 2.92393 & -0.54354 \\
$\mathrm{H}$ & -2.69647 & 3.88903 & -2.02090 \\
$\mathrm{H}$ & -0.37185 & -0.52557 & 1.05303 \\
& & & \\
\hline
\end{tabular}




\section{(S5) References}

(S1) (a) Lavallo, V.; Canac, Y.; Präsang, C.; Donnadieu, B.; Bertrand, G. Angew. Chem. Int. Ed. 2005, 44, 5705-5709; Angew. Chem. 2005, 117, 5851-5855. (b) Cui, H.; Cui, C. Chem. Asian J.2011, 6, 1138 1141.(c) Cowley, A. H.; Ebsworth, E. A. V.; Mehrotra, S. K.; Rankin, D. W. H.; Walkinshaw, M. D. J. Chem. Soc., Chem. Commun. 1982, 1099-1100. (d) Jutzi, P.; Kanne, D.; Hursthouse, M.; Howes, A. J. Chem. Ber.1988, 121, 1299-1305. (e) Sen, S. S.;Roesky, H. W.;Stern, D.;Henn, J.;Stalke, D.J. Am. Chem. Soc. 2010, 132, 1123-1126.

(S2) (a) Stalke, D. Chem. Soc. Rev.1998, 27, 171-178. (b) Kottke, T.; Stalke, D. J. Appl. Crystallogr. 1993, 26, 615-619.

(S3) Schulz, T.; Meindl, K.; Leusser, D.; Stern, D.; Graf, J.; Michaelsen, C.; Ruf, M.; Sheldrick, G. M.; Stalke, D. J. Appl. Crystallogr. 2009, 42, 885-891.

(S4) Bruker AXS Inc., SAINT v8.30C, WI, USA, Madison, 2013.

(S5) Krause, L.; Herbst-Irmer, R.; Sheldrick, G. M.; Stalke, D. J. Appl. Crystallogr. 2015, 48, 3-10.

(S6) Sheldrick, G. M. Acta Crystallogr. Sect. 2015, A71,3-8

(S7) (a) Sheldrick, G. M. Acta Crystallogr.2008, A64, 112 -122. (b) Sheldrick, G. M. Acta Crystallogr. 2015, $C 71,3-8$.

(S8) Gaussian09, Revision D.01, Frisch, M. J.; Trucks, G. W.; Schlegel, H. B.; Scuseria, G. E.; Robb, M. A.; Cheeseman, J. R.; Scalmani, G.; Barone, V.; Mennucci, B.; Petersson, G. A.; Nakatsuji, G. H.; Caricato, M.; Li, X.; Hratchian, H. P.; Izmaylov, A. F.; Bloino, J.; Zheng, G.; Sonnenberg, J. L.; Hada,M.; Ehara, M.; Toyota, K.; Fukuda, R.; Hasegawa, J.; Ishida, M.; Nakajima, T.; Honda, Y.; Kitao, O.; Nakai, H.; Vreven, T.; Montgomery, J. E. Peralta, F. Ogliaro, M. Bearpark, J. J. Heyd, E. Brothers, K. N. Kudin, V. N. Staroverov, R. Kobayashi, Jr. J. A.; Normand, J.; Raghavachari, K.; Rendell, A.; Burant, J. C.; Iyengar, S. S.; Tomasi, J.; Cossi, M.; Rega, N.; Millam, J. M.; Klene, M.; Knox, J. E.; Cross, J. B.; Bakken, V.; Adamo, C.; Jaramillo, J.; Gomperts, R.; Stratmann, R. E.; Yazyev, O.; Austin, A. J.; Cammi, R.; Pomelli, C.; Ochterski, J. W.; Martin, R. L.; Morokuma, K.; Zakrzewski, V. G.; Voth, G. A.; Salvador, P.; Dannenberg, J. J.; Dapprich, S.; Daniels, A. D.; Farkas, Ö.; Foresman, J. B.; Ortiz, J. V.; Cioslowski, J.; Fox, D. J. Gaussian, Inc., Wallingford CT, 2010.

(S9) Zhao, Y.; Schultz, N. E.; Truhlar, D. G.; J. Chem. Theory and Comput. 2006, 2, 364-382.

(S10) Schäfer, A.; Horn, H.; Ahlrichs, R. J. Chem. Phys. 1992, 97, 2571-2577.

(S11) Helgren, T. A.; Lipscomb, W. N. Chem. Phys. Lett.1977, 49 225-232. 
(S12) Weigend, F.; Ahlrichs, R. Phys. Chem. Chem. Phys. 2005, 7, 3297-3305.

(S13) Marenich, A. V.; Cramer, C. J.; Truhlar, D. G. J. Phys. Chem. B2009, 113, 6378-6396.

(S14) AIMAll (Version 13.11.04, Standard), Copyright (c) 1997-2013, T. A. Keith http://aim.tkgristmill.com

(S15) Bader, R. F. W. Chem. Rev. 1991, 94, 893-928.

(S16) (a) http://www.chemcraftprog.com. (b) Legault, C. Y. CYLView, 1.0b; Université de Sherbrooke: Canada, 2009; http://www.cylview.org.

(S17) Bader, R. F. W. Atoms in Molecules : A Quantum Theory. Oxford; New York: Clarendon Press 1990.

(S18) Niepötter, B.; Herbst-Irmer, R.; Kratzert, D.; Samuel, P. P.; Mondal, K. C.; Roesky, H. W.; Jerabek, P.; Frenking, G.; Stalke, D. Angew. Chem., Int. Ed. 2014, 53, 2766-2770.

(S19) Kocher, N.; Selinka, C.; Leusser, D.; Kost, D.; Kalikhman, I.; Stalke, D. Z. Anorg. Allg. Chem. 2004, 630, 1777-1793. 